Método de colocação polinomial para equações integro-diferenciais singulares:

convergência

Míriam Aparecida Rosa 


\title{
Método de colocação polinomial para equações integro-diferenciais singulares: convergência
}

\author{
Míriam Aparecida Rosa
}

Orientador: Prof. Dr. José Alberto Cuminato

Tese apresentada ao Instituto de Ciências Matemáticas e de Computação - ICMC-USP, como parte dos requisitos para obtenção do título de Doutor em Ciências - Ciências de Computação e Matemática Computacional. VERSÃO REVISADA 
Ficha catalográfica elaborada pela Biblioteca Prof. Achille Bassi e Seção Técnica de Informática, ICMC/USP, com os dados fornecidos pelo(a) autor(a)

Rosa, Míriam Aparecida
Método de colocação polinomial para equações
integro-diferenciais singulares / Miriam Aparecida
Rosa; orientador José Alberto Cuminato. -- São
Carlos, 2014 .
69 p.
Tese (Doutorado - Programa de Pós-Graduação em
Ciências de Computação e Matemática Computacional) --
Instituto de Ciências Matemáticas e de Computação,
Universidade de São Paulo, 2014.
1. Equações integro-diferenciais singulares. 2.
Método de Colocação Polinomial. 3. Convergência. 4.
Espaço de Besov ponderado. 5. Norma de Besov
ponderada. I. Cuminato, José Alberto, orient. II.
Título.




\section{Agradecimentos}

Aos meus pais, pelo grande apoio e compreensão nos momentos em que eu não pude estar presente, devido a preparação deste trabalho.

À toda minha família, pelo incentivo que recebi neste período.

Agradeço também aos amigos com quem convivi, pelas palavras de apoio recebidas em momentos difíceis e felizes, que tive durante o período desse trabalho.

Ao meu orientador Poti, pelas muitas coordenadas sugeridas, para que eu pudesse produzir este trabalho, e ao professor Valdemir, pelo grande incentivo e apoio sempre que lhe foi possível.

Ao CNPQ e à CAPES, pelo apoio financeiro. 


\section{Resumo}

Esta tese analisa o método de colocação polinomial, para uma classe de equações integro diferenciais singulares em espaços ponderados de funções contínuas e condições de fronteira não nulas. A convergência do método numérico em espaços com norma uniforme ponderada, é demonstrada, e taxas de convergências são determinadas, usando a suavidade dos dados das funções envolvidas no problema. Exemplos numéricos confirmam as estimativas. 


\section{Abstract}

This thesis analyses the polynomial collocation method, for a class of singular integrodifferential equations in weighted spaces of continuous functions, and non-homogeneous boundary conditions. Convergence of the numerical method, in weighted uniform norm spaces, is demonstrated and convergence rates are determined using the smoothness of the data functions involved in problem. Numerical examples confirm the estimates. 


\section{Sumário}

Contextualização $\quad 1$

1 Teoria das equações integrais singulares 3

1.1 Definições e teoremas principais . . . . . . . . . . . . . 3

1.2 O problema de valor de fronteira de Riemann-Hilbert para arcos abertos . 8

1.2.1 Solução do problema de Riemann-Hilbert homogêneo . . . . . . . . 9

1.2.2 Solução do problema de Riemann-Hilbert não-homogêneo . . . . . 12

1.2.3 Solução de equações integrais singulares com núcleo tipo Cauchy . . 13

1.2.4 Solução da equação dominante . . . . . . . . . . . . . . . 14

1.2.5 Solução da equação completa . . . . . . . . . . . . 16

2 Equações integro-diferenciais singulares $\quad 20$

2.1 Considerações iniciais . . . . . . . . . . . . . . . . . . 20

2.2 Método de colocação polinomial . . . . . . . . . . . . . . . 24

3 Espaço de Operadores $\quad 28$

3.1 Conceitos e teoremas principais . . . . . . . . . . . 28

3.2 Propriedades de mapeamento . . . . . . . . . . . . . . 30

3.3 Convergência do método de colocação polinomial . . . . . . . . . . . 34

4 Outros métodos numéricos do tipo colocação polinomial 49

4.1 Equação integro-diferencial singular analisada de outra forma . . . . . . . . 49

4.2 Convergência do método de colocação na norma uniforme . . . . . . . . . 51

4.3 Método Multhopp . . . . . . . . . . . . . . . . . . 52 
5 Exemplos Numéricos $\quad 54$

6 Conclusões $\quad 65$

$\begin{array}{ll}\text { Referências Bibliográficas } & 67\end{array}$ 


\section{Contextualização}

Desde a segunda metade do século XX, a teoria das equações integrais tem ganhado crescente importância, pelo fato de modelarem problemas físicos interessantes envolvendo aerodinâmica, mecânica de fraturas, acústica entre outros.

O clássico livro de Muskhelishvili [18], sintetiza os principais desenvolvimentos na teoria da equações integrais singulares (EIS). Usando a fórmula de Plemelj-Sokhotski, a EIS é reduzida a um problema equivalente ao problema de valor de fronteira de RiemannHilbert, e a partir daí se desenvolve a teoria de solução da EIS.

Uma classe de equações integrais que aparec com frequência em problemas práticos, são as equações integro-diferenciais, que são caracterizadas por apresentar derivadas da função incógnita sob o sinal de integração. Os primeiros autores a mencionarem a possibilidade de usar métodos numéricos para resolver EIS na solução de equações integrodiferenciais singulares (EIDS), foram Ioakimidis \& Theocaris [11]. Kalandiya em [13], modela uma série de problemas utilizando a equação de Prandtl

$$
\frac{\varphi(x)}{B(x)}-\frac{1}{2 \pi} f_{-1}^{1} \frac{\varphi^{\prime}(t)}{t-x} d t=f(x),
$$

com as condições de fronteira $\varphi(-1)=0$ e $\varphi(1)=0$, onde $B(x) \neq 0$ e $f(x)$ são Hölder contínuas. No entanto, EIDS com condições de fronteira não homogêneas aparecem na prática, como por exemplo, a equação da vela, estudada em [24].

$\mathrm{Na}$ literatura, uma gama enorme de métodos numéricos foram desenvolvidos para a solução de EIDS. Neste trabalho, será analisado o método de colocação polinomial (MCP), apresentado por Monegato \& Strozzi em [17], onde os autores provam a convergência desse método num espaço $L_{2}$ ponderado. Cuminato, em [6] e [7], prova a convergência do método na norma uniforme para a solução numérica de EIS com coeficientes constantes, e em [8] estende os resultados para o caso de coeficientes variáveis. Em [19], Nagamine \& Cuminato 
apresentam a análise de convergência para a solução numérica de EIDS com coeficientes constantes.

Um dos primeiros trabalhos que investigam a convergência uniforme ponderada de métodos de aproximação polinomial, para resolver EIS, foi apresentado por Capobianco, Junghanns, Luther \& Mastroianni em [3], e uma extensão foi apresentada em [12]. Em [4], [5] e [14] a análise de convergência do MCP, para resolver EIDS, foi realizada considerando condições de fronteira homogêneas, e em [4] a convergência desse método é provada na norma $L_{2}$ ponderada. Em [14], a mesma análise é realizada na norma $L_{1}$ ponderada, e em [5], na norma uniforme ponderada. A análise de convergência deste trabalho também é considerada na norma uniforme ponderada, no entanto, considerando a EIDS segundo um ponto de vista distinto do apresentado em [5] e condições de fronteira não homogêneas.

O principal objetivo desta tese é derivar uma estimativa da ordem de convergência do MCP, que depende da regularidade das funções que definem a EIDS.

Apresentamos primeiramente, uma breve introdução sobre a teoria das EIS de Cauchy, apresentando definições, lemas e principais teoremas, a relação entre as EIS e o problema de valor de fronteira de Riemann-Hilbert, e métodos de solução.

No Capítulo 2, apresentamos a EIDS objeto deste estudo, sua redução a uma EIS, a representação em forma de operadores e aplicação do MCP.

No Capítulo 3, apresentamos um espaço ponderado de funções contínuas, ao qual pertencem os coeficientes da EIDS proposta, as propriedades de mapeamento dos operadores definidos no Capítulo 2, a prova da convergência do MCP, e uma estimativa para a taxa de convergência do MCP.

No Capítulo 4, analisamos a EIDS proposta no Capítulo 1 segundo o ponto de vista proposto em [5], mostramos a estimativa para a taxa de convergência do MCP na norma uniforme apresentada em [19], apresentamos o método Multhopp, e comentamos suas restrições quanto ao tratamento da EIDS.

No Capítulo 5, apresentamos alguns exemplos de EIDS resolvidos com o MCP. Fazemos uma análise dos dados do problema para deduzir uma estimativa para a ordem de convergência. Apresentamos os resultados numéricos obtidos com a aplicação do MCP, que comprovam essas estimativas. 


\section{Capítulo 1}

\section{Teoria das equações integrais singulares}

Neste capítulo, apresentamos os principais conceitos da teoria das EIS e sua relação com o problema de valor de fronteira de Riemann-Hilbert. Utilizamos esta teoria no tratamento das EIDS.

Apresentamos aqui, apenas os principais conceitos e resultados básicos, necessários para uma breve revisão bibliográfica do assunto. Todos os detalhes desta teoria, são apresentados, discutidos e demonstrados no clássico livro sobre o tema: Singular Integral Equations de Muskhelishvili, [18].

\subsection{Definições e teoremas principais}

Definição 1.1.1. Um contorno suave L, é uma curva no plano complexo representada na forma

$$
\mathrm{L}(s):=(x(s), y(s)), \quad a \leq s \leq b,
$$

onde $x(s)$ e $y(s)$ são funções contínuas no intervalo de definição, satisfazendo as seguintes propriedades:

1) Suas derivadas de primeira ordem $x^{\prime}(s)$ e $y^{\prime}(s)$, respectivamente, são contínuas ao longo de $\mathrm{L}$ e não simultaneamente nulas;

2) Se $s_{1}, s_{2} \in(a, b), x\left(s_{1}\right)=x\left(s_{2}\right)$ e $y\left(s_{1}\right)=y\left(s_{2}\right)$ somente se $s_{1}=s_{2}$.

3) Se $x(a)=x(b)$ e $y(a)=y(b)$, L é um contorno fechado. 
Faremos referência aos pontos a e b como os extremos de $\mathrm{L}$, e em alguns casos indicaremos $\mathrm{L}$ por $\mathrm{L}=a b$.

Definição 1.1.2. Definimos a orientação positiva de L, como sendo aquela em que L é percorrido no sentido de a para $b$.

Definição 1.1.3. Seja $\varphi$ uma função real ou complexa definida sobre L. Dizemos que $\varphi$ satisfaz a condição de Hölder em $\mathrm{L}$, se para quaisquer $t_{1}, t_{2}$, pontos de $\mathrm{L}$,

$$
\left|\varphi\left(t_{2}\right)-\varphi\left(t_{1}\right)\right| \leq C\left|t_{2}-t_{1}\right|^{\mu}
$$

onde $C$ é constante positiva denominada constante de Hölder e $0<\mu \leq 1$ é denominado indice de Hölder. Nestas condições, dizemos que $\varphi$ satisfaz a condição $\mathcal{H}^{\mu}(\mathrm{L})$, ou simplesmente, satisfaz a condição $\mathcal{H}(\mathrm{L})$, quando não houver necessidade de menção ao indice $\mu$.

Definição 1.1.4. Sejam L e L' contornos suaves, definidos nos intervalos $[a, b]$ e $\left[a^{\prime}, b^{\prime}\right]$, respectivamente, para $\left[a^{\prime}, b^{\prime}\right] \subset[a, b]$ mas $a^{\prime} \neq a, b^{\prime} \neq b$. Suponha que a função $\varphi(t)$ satisfaz a condição $\mathcal{H}\left(\mathrm{L}^{\prime}\right)$, e que nos extremos de $\mathrm{L}$ e próxima a eles, $\varphi$ seja da forma:

$$
\varphi(t)=\frac{\varphi^{*}(t)}{(t-c)^{\alpha}}, \quad 0 \leq \alpha<1
$$

onde $\varphi^{*}(t)$ satisfaz a condição $\mathcal{H}(\mathrm{L})$ e c representa um dos extremos de $\mathrm{L}$. Neste caso, dizemos que $\varphi$ satisfaz a condição $\mathcal{H}^{*}(\mathrm{~L})$.

Considere um contorno suave $\mathrm{L}=a b$. Sejam $x$ um ponto de $\mathrm{L}$ não coincidindo com os extremos, $\gamma$ um disco centrado em $x$ de raio $\delta, l$ a intersecção entre $\mathrm{L}$ e $\gamma$, e $t_{1}, t_{2}$ os extremos de $l$. Defina como orientação positiva de $l$, a de $t_{1}$ para $t_{2}$, conforme a Figura 1.1.

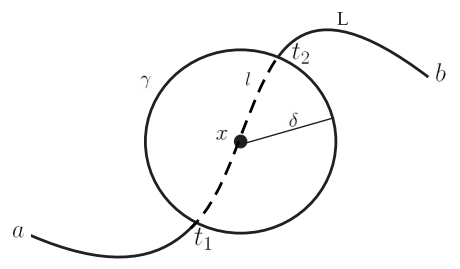

Figura 1.1: Contorno L $-l$ 
À vizinhança de $x$, dividida nas regiões de $\gamma$ à direita e à esquerda de $l$ quando percorrido no sentido positivo, nos referimos como vizinhanças à direita e à esquerda de $x$, e as denotaremos por $S^{-}$e $S^{+}$, respectivamente. Se L for um contorno fechado, denotamos as regiões à direita e à esquerda de L, quando percorrido no sentido anti-horário, por $S^{-}$ e $S^{+}$respectivamente, conforme a Figura 1.2.
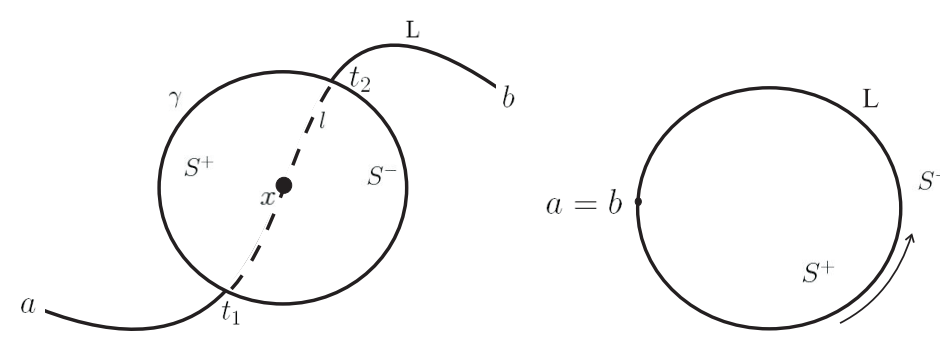

Figura 1.2: Regiões definidas pela curva L

Definição 1.1.5. O valor principal da integral de Cauchy de $\varphi$ no ponto $x$ de $\mathrm{L}$, é definido por:

$$
f_{\mathrm{L}} \frac{\varphi(t)}{t-x} d t:=\lim _{\delta \rightarrow 0} \int_{\mathrm{L}-l} \frac{\varphi(t)}{t-x} d t
$$

quando o limite existir.

Definição 1.1.6. Sejam $x$ um ponto arbitrário de L, que não coincida com seus extremos, e $\Phi(z)$ uma função contínua em todo ponto z da vizinhança de $x$. Dizemos que $\Phi(z)$ é contínua à esquerda (resp. à direita) de L, se $\Phi(z)$ tende a um limite definido $\Phi^{+}(x)$ (resp. $\Phi^{-}(x)$ ), quando z tende a $x$, para z um ponto da vizinhança à esquerda (resp. à direita) de $x$.

Apresentamos a seguir, um teorema que será crucial para obtenção dos resultados seguintes.

Teorema 1.1.7. Sejam L um contorno suave, z um ponto do plano e $\varphi$ uma função que satisfaz a condição $\mathcal{H}(\mathrm{L})$. Então, a função

$$
\Phi(z)=\frac{1}{2 \pi i} \int_{\mathrm{L}} \frac{\varphi(t)}{t-z} d t
$$

é contínua à esquerda e à direita de $\mathrm{L}$, exceto possivelmente nos extremos para os quais $\varphi(x) \neq 0$. 
A demonstração deste teorema, pode ser encontrada em [18, p. 38].

Observe que o caso em que $z \in \mathrm{L}$, implica que $\Phi(x)$ é contínua sobre L, exceto possivelmente, nos extremos para os quais $\varphi(x) \neq 0$.

Definição 1.1.8. Sejam L um contorno suave, $\varphi$ uma função que satisfaz a condição $\mathcal{H}(\mathrm{L}), z \notin \mathrm{L}$, e $x$ um ponto de $\mathrm{L}$ que não coincida com um dos seus extremos, caso neles $\varphi(x) \neq 0$. Definimos os limites à esquerda e à direita de L a partir da função

$$
\Phi(z)=\frac{1}{2 \pi i} \int_{\mathrm{L}} \frac{\varphi(t)}{t-z} d t
$$

respectivamente por:

$$
\Phi^{+}(x)=\lim _{z \rightarrow x} \Phi(z), \quad z \in S^{+} \quad e \quad \Phi^{-}(x)=\lim _{z \rightarrow x} \Phi(z), \quad z \in S^{-} .
$$

Teorema 1.1.9. (Fórmulas de Plemelj-Sokhotski) Seja $\Phi(z)$ como na Definição 1.1.8. Então

$$
\Phi^{+}(x)=\frac{1}{2} \varphi(x)+\frac{1}{2 \pi i} f_{\mathrm{L}} \frac{\varphi(t)}{t-x} d t
$$

$e$

$$
\Phi^{-}(x)=-\frac{1}{2} \varphi(x)+\frac{1}{2 \pi i} f_{\mathrm{L}} \frac{\varphi(t)}{t-x} d t
$$

A demonstração deste teorema pode ser encontrada em [18, p. 42].

Observe que somando e subtraindo as fórmulas de Plemelj-Sokhotski, obtemos

$$
\begin{aligned}
\Phi^{+}(x)+\Phi^{-}(x) & =\frac{1}{\pi i} f_{\mathrm{L}} \frac{\varphi(t)}{t-x} d t, \\
\Phi^{+}(x)-\Phi^{-}(x) & =\varphi(x) .
\end{aligned}
$$

Em [18, p. 46], encontra-se a demonstração do teorema seguinte, feita considerando o Teorema 1.1.9.

Teorema 1.1.10. (Teorema de Plemelj-Privalov) Suponha $\Phi$ como na Definição 1.1.8. Se $\varphi(x)$ satisfaz a condição $\mathcal{H}^{\mu}(\mathrm{L})$, então $\Phi^{+}(x)$ e $\Phi^{-}(x)$ satisfazem a condição $\mathcal{H}^{\mu}(\mathrm{L})$ quando $\mu<1$, e $\mathcal{H}^{1-\varepsilon}(\mathrm{L})$ quando $\mu=1$, com $\varepsilon>0$ suficientemente pequeno, exceto possivelmente em uma vizinhança arbitrariamente pequena dos extremos, para os quais $\varphi(x) \neq 0$. 
Destes dois últimos teoremas, segue que:

Teorema 1.1.11. Seja $\Phi$ como na Definição 1.1.8. Se $\varphi(x)$ satisfaz a condição $\mathcal{H}^{\mu}(\mathrm{L})$, então $\Phi(x)$ satisfaz $\mathcal{H}^{\mu}(\mathrm{L})$ quando $\mu<1$ e $\mathcal{H}^{1-\varepsilon}(\mathrm{L})$ quando $\mu=1$, com $\varepsilon>0$ suficientemente pequeno, exceto possivelmente em uma vizinhança arbitrariamente pequena dos extremos, para os quais $\varphi(x) \neq 0$.

A demonstração deste teorema, pode ser encontrada em [18, p. 46].

Apresentamos a seguir, alguns conceitos associados ao problema de valor de fronteira de Riemann-Hilbert, que enunciaremos na próxima seção.

Definição 1.1.12. Sejam L um contorno suave, e $\Phi(z)$ uma função contínua em L $e$ holomorfa no plano, exceto nos pontos de L. Suponha também que $\Phi(z)$ seja contínua à esquerda e à direita de $\mathrm{L}$, exceto possivelmente nos extremos, nos quais satisfaz a condição

$$
|\Phi(z)| \leq \frac{C}{|z-c|^{\alpha}}, \quad 0 \leq \alpha<1
$$

onde c representa um extremo genérico e $C$ é uma constante positiva. Então $\Phi(z)$ é dita ser seccionalmente holomorfa com linha de descontinuidade L.

Definição 1.1.13. Se na expansão

$$
\Phi(z)=\sum_{j=-\infty}^{j=+\infty} a_{j} z^{j},
$$

de uma função $\Phi(z)$ na vizinhança de um ponto no infinito, existir apenas um número finito de termos com potências positivas de $z$, dizemos que $\Phi(z)$ possui grau finito no infinito.

Teorema 1.1.14. Seja $\varphi(x)$ uma função que satisfaz a condição $\mathcal{H}^{\mu}(\mathrm{L})$. Se $\Phi(z)$ é uma função seccionalmente holomorfa, exceto possivelmente para $z=\infty$, que possui grau finito no infinito e satisfaz a condição de fronteira

$$
\Phi^{+}(x)-\Phi^{-}(x)=\varphi(x), \quad x \in \mathrm{L},
$$

então

$$
\Phi(z)=\frac{1}{2 \pi i} \int_{\mathrm{L}} \frac{\varphi(t)}{t-z} d t+P_{k}(z),
$$

onde $P_{k}(z)$ é um polinômio arbitrário de grau no máximo $k$. 
Demonstração: A igualdade (1.1) pode ser obtida diretamente das fórmulas de PlemeljSokhotski, e sua unicidade, exceto pelo termo $P_{k}(z)$, pode ser facilmente demonstrada.

Suponha que exista uma outra função $\Psi(z)$ que também satisfaça as condições do teorema. A função $\Upsilon(z)=\Phi(z)-\Psi(z)$ é tal que

$$
\Upsilon^{+}(x)-\Upsilon^{-}(x)=0
$$

Sendo $\Upsilon(z)$ uma função holomorfa no plano, e que se anula no infinito, necessariamente $\Upsilon(z)=0$.

Todos os resultados apresentados até aqui continuam válidos para os casos em que $\varphi \in \mathcal{H}^{*}(\mathrm{~L})$. Este caso, é discutido em [18].

O problema de valor de fronteira de Riemann-Hilbert que enunciamos a seguir, mostra claramente sua conexão com a resolução da integral singular do Teorema 1.1.14.

\subsection{O problema de valor de fronteira de Riemann-Hilbert para arcos abertos}

\section{Problema de Riemann-Hilbert:}

Sejam L um contorno suave e $G(x)$ uma função que não se anula em ponto algum de $\mathrm{L}$ e satisfaz a condição $\mathcal{H}(\mathrm{L})$. Encontrar uma função seccionalmente holomorfa $\Phi(z)$ com grau finito no infinito, que satisfaça a condição de fronteira

problema homogêneo: $\Phi^{+}(x)=G(x) \Phi^{-}(x)$ em L.

problema não-homogêneo: $\Phi^{+}(x)=G(x) \Phi^{-}(x)+g(x)$ em L,

onde $g(x)$ satisfaz a condição $\mathcal{H}(\mathrm{L})$.

Apresentamos a seguir, a resolução do problema de valor de fronteira de RiemannHilbert. Nos problemas de nosso interesse, o contorno L é um arco aberto. Tendo em vista a resolução de equações integrais singulares via problema de Riemann-Hilbert, apresentamos os problemas de Riemann-Hilbert homogêneo e não-homogêneo para arcos abertos. Os casos em que L consiste de um conjunto finito de contornos suaves fechados que não se 
intersectam, é considerado e discutido em [18]. Primeiramente, consideramos o problema de valor de fronteira de Riemann-Hilbert homogêneo, e em seguida, com o auxílio da solução do problema homogêneo, mostramos a resolução do problema não-homogêneo.

\subsubsection{Solução do problema de Riemann-Hilbert homogêneo}

No que se segue, vamos considerar que:

1) L é a união finita de arcos abertos suaves $\mathrm{L}_{j}, 1 \leq j \leq p$, não se intercectando, e com uma direção (positiva) definida. Indicaremos seus extremos por: $a_{j}, b_{j}$, sendo a direção positiva de $\mathrm{L}_{j}$ definida de $a_{j}$ para $b_{j}$,

2) $\varphi$ satisfaz a condição $\mathcal{H}^{*}(\mathrm{~L})$.

3) Definimos

$$
\Delta(z)=\frac{1}{2 \pi i} \int_{\mathrm{L}} \frac{\ln G(t)}{t-z} d t
$$

tomando para $\ln G(t)$ algum ramo desta função multivalente que varia continuamente sobre cada $\mathrm{L}_{j}$.

Admitindo que estas condições estão satisfeitas, pelas equações de Plemelj-Sokhotski, a função $\Phi(z)=e^{\Delta(z)}$ satisfaz a igualdade

$$
\Phi^{+}(x)=G(x) \Phi^{-}(x) \text { em L. }
$$

No entanto, $e^{\Delta(z)}$ pode não ser seccionalmente holomorfa por não satisfazer a condição

$$
\left|e^{\Delta(z)}\right|<\frac{C}{\left|z-c_{j}\right|^{\alpha}} .
$$

Para contornar este problema, veja que $\ln G(t)$ satisfaz a condição $\mathcal{H}(\mathrm{L})$, e neste caso, foi demonstrado em [18, p. 230], que próximo aos extremos $c_{j}$, a função $\Delta(z)$ possui a forma

$$
\Delta(z)= \pm \frac{\ln \left[G\left(c_{j}\right)\right]}{2 \pi i} \ln \frac{1}{z-c_{j}}+\Delta^{*}(z)
$$

onde o sinal acima é positivo se $c_{j}=a_{j}$ e negativo se $c_{j}=b_{j}$. A função $\Delta^{*}(z)$ satisfaz a condição $\mathcal{H}(\mathrm{L})$ próxima a $c_{j}$ se $z \in \mathrm{L}$, e para $z$ não pertencente a $\mathrm{L}, \Delta^{*}(z)$ é uma função limitada, tendo limite definido quando $z \rightarrow c_{j}$. Sendo assim, definindo

$$
\gamma_{j}+i \delta_{j}=\mp \frac{\ln G\left(c_{j}\right)}{2 \pi i}
$$


com o sinal acima negativo se $c_{j}=a_{j}$ e positivo se $c_{j}=b_{j}$, e $\gamma_{j}$ e $\delta_{j}$ constantes reais, podemos escrever

$$
e^{\Delta(z)}=\left(z-c_{j}\right)^{\gamma_{j}+i \delta_{j}} \Omega(z)
$$

onde $\Omega(z)$ é função limitada que não se anula.

Selecione inteiros $\lambda_{j}$, tais que

$$
-1<\gamma_{j}+\lambda_{j}<1
$$

e defina

$$
\Pi(z)=\prod_{j=1}^{2 p}\left(z-c_{j}\right)^{\lambda_{j}}
$$

Então a função

$$
X(z)=\Pi(z) e^{\Delta(z)}
$$

satisfaz a condição (1.5), e por (1.7) $X(z)$ é seccionalmente holomorfa. Sendo assim, $X(z)$ é uma solução particular do problema de Riemann-Hilbert para arcos abertos, e será chamada solução fundamental, assim como $c X(z)$, para $c$ uma constante não nula.

No entanto, esta solução só está completamente definida se $\gamma_{j}$ for inteiro, pois caso contrário, a escolha dos valores de $\lambda_{j}$ que satisfazem a condição (1.7) não é única. Se $\gamma_{j}$ for inteiro, $\lambda_{j}$ é determinado unicamente por

$$
\lambda_{j}=-\gamma_{j}
$$

Definição 1.2.1. Aos extremos $c_{j}$ para os quais $\gamma_{j}$ é inteiro denominamos extremos especiais. Aos demais denominamos extremos não-especiais.

Definição 1.2.2. Sejam $c_{j}, 1 \leq j \leq m$, todos os extremos não-especiais de uma solução $\Phi(z)$ do problema de Riemann-Hilbert (1.5). Se nos extremos $c_{j}, 1 \leq j \leq q$, $\Phi(z)$ for limitada, dizemos que $\Phi(z)$ é uma solução de classe $h\left(c_{1}, \ldots, c_{q}\right)$. Se $q=m$, dizemos simplesmente que $\Phi(z)$ é de classe $h_{m}$ e se $q=0$, que $\Phi(z)$ é de classe $h_{0}$.

Tomando os limites à esquerda e à direita de $X(z)$ sobre $\mathrm{L}$, e aplicando as fórmulas de Plemelj-Sokhotski, obtemos

$$
X^{+}(x)=\sqrt{G(x)} X(x) \quad X^{-}(x)=\frac{X(x)}{\sqrt{G(x)}},
$$


donde

$$
X(x)=\prod_{j=1}^{2 p}\left(x-c_{j}\right)^{\alpha_{j}} \Omega(x),
$$

sendo $\alpha_{j}=\gamma_{j}+i \delta_{j}+\lambda_{j}$, e $\Omega(x)$ uma função que satisfaz a condição $\mathcal{H}(\mathrm{L})$ e que não se anula em L. Portanto, se a função $X(z)$ pertence à classe $h\left(c_{1}, \ldots, c_{q}\right), X(z)$ se anula nos extremos $c_{1}, \ldots, c_{q}$, e não se anula em outras partes do plano. Além disso, nos extremos especiais $X(z)$ é limitada.

Definição 1.2.3. Ao inteiro

$$
\varkappa=-\sum_{j=1}^{2 p} \lambda_{j},
$$

denominamos índice do problema de Riemann-Hilbert em uma dada classe de soluções $h\left(c_{1}, \ldots, c_{q}\right)$.

Por (1.8), conclui-se que o grau da solução $X(z)$ no infinito é $-\varkappa$.

Teorema 1.2.4. Uma função $\Phi(z)$ é solução do problema de Riemann-Hilbert (1.5) em uma dada classe, se e somente se

$$
\Phi(z)=X(z) P(z)
$$

onde $P(z)$ é um polinômio arbitrário e $X(z)$ é uma solução fundamental do problema.

$\underline{\text { Demonstração: }}$ Suponha que $\Phi(z)$ seja solução do problema de Riemann-Hilbert (1.5) em uma dada classe. Por hipótese,

$$
\Phi^{+}(x)=G(x) \Phi^{-}(x) \text { e } X^{+}(x)=G(x) X^{-}(x) \text { em L },
$$

sendo que $X^{+}(x), X^{-}(x) \neq 0$, exceto nos extremos e para um ponto no infinito. Portanto,

$$
\frac{\Phi(x)^{+}}{X^{+}(x)}=\frac{\Phi^{-}(x)}{X^{-}(x)}
$$

e consequentemente, $\Phi(x) / X(x)$ é holomorfa no plano, e próxima aos extremos poderá tornar-se infinita, com grau menor que um. Como $\Phi(z)$ possui grau finito no infinito, $\Phi(x) / X(x)$ é um polinômio.

Por outro lado, se (1.11) é válido, obtemos

$$
\Phi^{+}(x)=X^{+}(x) P(x) \text { e } \Phi^{-}(x)=X^{-}(x) P(x) .
$$


Como $X^{+}(x)=G(x) X^{-}(x)$, tem-se

$$
\Phi^{+}(x)=G(x) \Phi^{-}(x)
$$

Observe que para $\Phi(z)$ ser limitada próxima a um extremo não especial $c$, o polinômio $P(z)$ deve possuir o termo $(z-c)$, e neste caso, $\Phi(z)=0$ para $z=c$.

Segue de (1.11), que no infinito o grau $\Phi(z)=-\varkappa+k$, onde $k$ é o grau do polinômio $P(z)$. Portanto, o menor grau possível de uma solução no infinito é $-\varkappa$, e neste caso, ela é uma solução fundamental.

\subsubsection{Solução do problema de Riemann-Hilbert não-homogêneo}

Seja $\Phi(z)$ uma solução de classe $h\left(c_{1}, \ldots, c_{q}\right)$ do problema de Riemann-Hilbert não-homogêneo

$$
\Phi^{+}(x)=G(x) \Phi^{-}(x)+g(x) \mathrm{em} \mathrm{L}
$$

onde $G(x)$ e $g(x)$ satisfazem a condição $\mathcal{H}(\mathrm{L})$ e $G(x)$ não se anula em ponto algum de L. Seja também $X(z)$ a solução fundamental, de mesma classe, do problema de Riemann-Hilbert homogêneo associado ao problema (1.12)

$$
\Phi^{+}(x)=G(x) \Phi^{-}(x) e m \mathrm{~L},
$$

a qual denominamos função fundamental de classe $h\left(c_{1}, \ldots, c_{q}\right)$ do problema (1.12). Então

$$
\frac{\Phi^{+}(x)}{X^{+}(x)}-\frac{G(x)}{X^{+}(x)} \Phi^{-}(x)=\frac{g(x)}{X^{+}(x)} \Rightarrow \frac{\Phi^{+}(x)}{X^{+}(x)}-\frac{\Phi^{-}(x)}{X^{-}(x)}=\frac{g(x)}{X^{+}(x)} .
$$

Por suposição, $\Phi(z)$ e $X(z)$ são limitadas próximas aos extremos $c_{1}, \ldots, c_{q}$ e portanto,

$$
\left|\frac{\left.\Phi^{(} z\right)}{X(z)}\right|<\frac{C}{\left|z-c_{j}\right|^{\alpha}}, \quad 1 \leq j \leq q .
$$

Sendo assim, a função $\Phi(z) / X(z)$ é seccionalmente holomorfa e possui grau finito no infinito. De acordo com o Teorema 1.1.14,

$$
\Phi(z)=\frac{X(z)}{2 \pi i} \int_{L} \frac{g(t)}{X^{+}(t)(t-z)} d t+X(z) P_{k}(z)
$$

onde $P_{k}(z)$ é um polinômio arbitrário de grau no máximo $k$. 
Teorema 1.2.5. A solução $\Phi(z)$ do problema de Riemann-Hilbert não-homogêneo (1.12), de classe $h\left(c_{1}, \ldots, c_{q}\right)$, que se anula no infinito, é dada por:

- $S e \varkappa \geq 0$,

$$
\Phi(z)=\frac{X(z)}{2 \pi i} \int_{L} \frac{g(t)}{X^{+}(t)(t-z)} d t+X(z) P_{\varkappa-1}(z),
$$

onde $X(z)$ é a função fundamental de classe $h\left(c_{1}, \ldots, c_{q}\right)$ e indice $\varkappa$ do problema (1.12), $P_{\varkappa-1}(z)$ é um polinômio arbitrário de grau no máximo $\varkappa-1$ e $P_{\varkappa-1} \equiv 0$ para $\varkappa=0$.

- Se $\varkappa<0$, a solução só existe se

$$
\int_{L} \frac{t^{j} g(t)}{X^{+}(t)} d t=0, \quad j=0,1, \cdots,-\varkappa-1 .
$$

Se estas condições forem satisfeitas, então $\Phi(z)$ é dada por (1.13) com $P_{\varkappa-1} \equiv 0$.

A demonstração deste teorema pode ser encontrada em [6], Seção 81.

\subsubsection{Solução de equações integrais singulares com núcleo tipo Cauchy}

Apresentamos a seguir, algumas definições e resultados necessários ao desenvolvimento da teoria da resolução de EIS.

Definição 1.2.6. Seja a equação integral

$$
a(x) \varphi(x)+\frac{1}{\pi i} \int_{\mathrm{L}} \frac{\mathbf{K}(x, t) \varphi(x)}{t-x} d t=f(x),
$$

onde $f(t)$ é dada e $\varphi(t)$ é desconhecida. Se as seguintes condições,

1) as funções a $(t)$ e $\mathbf{K}(x, t)$ satisfazem a condição $\mathcal{H}(\mathrm{L})$, no caso de $\mathbf{K}(x, t)$ em ambas as variáveis, e $f(x)$ satisfaz a condição $\mathcal{H}^{*}(\mathrm{~L})$,

2) L consiste da união finita de arcos suaves não se interceptando,

3) as funções $a(x)$ e $b(x)$ são tais que $a^{2}(x)-b^{2}(x) \neq 0$,

forem satisfeitas, a equação acima será chamada equação integral singular com núcleo do tipo Cauchy ou simplesmente equação integral singular. 
Podemos reescrever a equação (1.14) de uma forma mais conveniente, como:

$$
a(x) \varphi(x)+\frac{b(x)}{\pi i} \int_{\mathrm{L}} \frac{\varphi(t)}{t-x} d t+\int_{\mathrm{L}} l(x, t) \varphi(t) d t=f(x)
$$

onde $b(x)=K(x, x)$ e $l(x, t)=[K(x, t)-K(x, x)] /[\pi i(t-x)]$.

Definição 1.2.7. A equação

$$
a(x) \varphi(x)+\frac{b(x)}{\pi i} \int_{\mathrm{L}} \frac{\varphi(t)}{t-x} d t=f(x)
$$

é denominada equação dominante correspondente à equação completa (1.14), e os termos a $(x)$ e $b(x)$ são os coeficientes da equação dominante.

\subsubsection{Solução da equação dominante}

Primeiramente investigamos a resolução da equação dominante, e em seguida, de posse desta solução, podemos resolver a equação completa.

Lema 1.2.8. Considere a equação (1.15) e as condições 1,2 e 3 da Definição 1.2.6 verdadeiras. Suponha que

$$
\Phi(z)=\frac{1}{2 \pi i} \int_{\mathrm{L}} \frac{\varphi(t)}{t-z} d t
$$

onde $\varphi(t)$ é a solução de (1.15), e satisfaz a condição $\mathcal{H}^{*}(\mathrm{~L})$. Então $\Phi(z)$ é solução do problema de Riemann-Hilbert

$$
\Phi(x)^{+}=\frac{a(x)-i b(x)}{a(x)+i b(x)} \Phi(x)^{-}+\frac{f(x)}{a(x)+i b(x)},
$$

que se anula no infinito.

Demonstração: Aplicando as fórmulas de Plemelj-Sokhotski para $\Phi(z)$, e substituindo em (1.15), obtemos (1.16).

Pelo o Teorema 1.1.14, obtemos

$$
\Phi(z)=\frac{1}{2 \pi i} \int_{\mathrm{L}} \frac{\varphi(t)}{t-z} d t+P_{k}(z)
$$

onde $P_{k}(z)$ é um polinômio arbitrário de grau no máximo $k$. De acordo com o Teorema 1.2.5, a solução de classe $h\left(c_{1}, \cdots, c_{q}\right)$ do problema de Riemann-Hilbert: 
Encontrar uma função $\Phi(z)$ seccionalmente holomorfa, com grau finito no infinito $e$ que satisfaça a condição de fronteira (1.16), onde $a(x)$ e b(x) satisfazem a condição $\mathcal{H}(\mathrm{L})$ e $a^{2}(x)-b^{2}(x) \neq 0$, é dada por:

- $S e \varkappa \geq 0$,

$$
\Phi(z)=\frac{X(z)}{2 \pi i} \int_{\mathrm{L}} \frac{f(t)}{[a(t)+i b(t)] X^{+}(t)(t-z)} d t+X(z) P_{\varkappa-1}(z)
$$

onde $X(z)$ é a função fundamental de classe $h\left(c_{1}, \cdots, c_{q}\right)$ e índice $\varkappa$, e $P_{\varkappa-1}(z)$ é um polinômio arbitrário de grau no máximo $\varkappa-1$, sendo que $P_{\varkappa-1} \equiv 0$ para $\varkappa=0$.

- $S e \varkappa<0$, a solução só existe se

$$
\int_{\mathrm{L}} \frac{t^{j} f(t)}{[a(t)+i b(t)] X^{+}(t)} d t=0, \quad j=0,1, \cdots,-\varkappa-1 .
$$

Se estas condições forem satisfeitas, $\Phi(z)$ é dada por (1.17) $\operatorname{com} P_{\varkappa-1} \equiv 0$.

Notação. Denotamos a função fundamental de classe $h\left(c_{1}, \cdots, c_{q}\right)$ do problema de Riemann-Hilbert (1.16), por $Z(x)$, conforme definida em (1.8), e por $r(x)$ a função $\sqrt{a^{2}(x)+b^{2}(x)}$.

Teorema 1.2.9. Nas condições do Lema 1.2.8, a solução geral da equação (1.15) é dada por

$$
\varphi(x)= \begin{cases}A^{*} f-\frac{2 i b(x) Z(x)}{r(x)} P_{\varkappa-1}(x) \quad \text { se } \quad \varkappa \geq 0 \\ A^{*} f \quad \text { se } \quad \varkappa<0 \text { e } \\ & \int_{L} \frac{t^{j} f(t)}{r(t) Z(t)} d t=0, \quad j=0,1, \ldots,-\varkappa-1,\end{cases}
$$

onde

$$
A^{*} f=\frac{a(x)}{r^{2}(x)} f(x)-\frac{b(x) Z(x)}{\pi i r(x)} \int_{L} \frac{f(t) d t}{r(t) Z(t)(t-x)} .
$$

$\underline{\text { Demonstração: }}$ Tomando os limites à esquerda e à direita de (1.17) e substituindo em

$$
\varphi(x)=\Phi^{+}(x)-\Phi^{-}(x),
$$


obtemos:

$$
\begin{aligned}
\varphi(x) & =\frac{X^{+}(x)+X^{-}(x)}{2[a(x)+i b(x)] X^{+}(x)} f(x)+\frac{X^{+}(x)-X^{-}(x)}{2 \pi i} \int_{\mathrm{L}} \frac{f(t) d t}{[a(t)+i b(t)] X^{+}(t)(t-x)}+ \\
& +\left[X^{+}(x)-X^{-}(x)\right] P_{\varkappa-1} .
\end{aligned}
$$

Como $X(z)$ é solução fundamental do problema de Riemann-Hilbert homogêneo

$$
\Phi^{+}(x)=\left[\frac{a(x)-i b(x)}{a(x)+i b(x)}\right] \Phi^{-}(x)=G(x) \Phi^{-}(x),
$$

associado ao problema (1.16) e de mesma classe, por (1.9)

$$
X^{+}(x)=\sqrt{G(x)} Z(x) \text { e } X^{-}(x)=\frac{1}{\sqrt{G(x)}} Z(x),
$$

o que implica em

$$
\begin{aligned}
& {[a(x)+i b(x)] X^{+}(x)=\frac{r(x) X^{+}(x)}{\sqrt{G}(x)}=r(x) Z(x)} \\
& X^{+}(x)+X^{-}(x)=\frac{2 a(x) Z(x)}{r(x)} \text { e } \quad X^{+}(x)-X^{-}(x)=-\frac{2 i b(x) Z(x)}{r(x)}
\end{aligned}
$$

e aplicando as equações (1.20) em (1.19), obtemos

$$
\varphi(x)=A^{*} f-\frac{2 i b(x) Z(x)}{r(x)} P_{\varkappa-1}(x), P_{\varkappa-1}(x) \equiv 0 \text { se } \varkappa \leq 0 .
$$

Se $\varkappa<0$, pelo Teorema 1.2.5, para que a solução exista as condições

$$
\int_{\mathrm{L}} \frac{t^{j} f(t)}{r(t) Z(t)} d t=0, \quad j=0,1, \cdots,-\varkappa-1,
$$

devem ser satisfeitas.

\subsubsection{Solução da equação completa}

Seja a EIS

$$
\mathbf{A} \varphi=a(x) \varphi(x)+\frac{b(x)}{\pi} \int_{\mathrm{L}} \frac{\varphi(t)}{t-x} d t+\int_{\mathrm{L}} l(x, t) \varphi(t) d t=f(x),
$$

como na Definição 1.2.6. Definindo

$$
\begin{aligned}
\mathbf{A}^{\circ} \varphi & \equiv a(x) \varphi(x)+\frac{b(x)}{\pi} \int_{\mathrm{L}} \frac{\varphi(t)}{t-x} d t \mathrm{e} \\
\mathbf{a} \varphi & \equiv \int_{\mathrm{L}} l(x, t) \varphi(t) d t
\end{aligned}
$$


podemos escrever (1.21) como:

$$
\mathbf{A} \varphi=\mathbf{A}^{\circ} \varphi+\mathbf{a} \varphi=f \quad \Rightarrow \quad \mathbf{A}^{\circ} \varphi=f-\mathbf{a} \varphi
$$

Se o lado direito desta última equação for conhecido, pela seção anterior podemos resolver esta equação. Como $f-\mathbf{a} \varphi$ satisfaz a condição $\mathcal{H}^{*}(\mathrm{~L})$, a solução da equação completa é dada por

$$
\varphi(x)=A^{*}[f-\mathbf{a} \varphi]-\frac{2 i b(x) Z(x)}{r(x)} P_{\varkappa-1}(x)
$$

para $A^{*} f, Z(x)$ e $P_{\varkappa-1}(x)$ como no Teorema 1.2.9. Se $\varkappa<0$, para que a solução exista, as seguintes condições devem ser satisfeitas:

$$
\int_{\mathrm{L}} \frac{t^{j}[f(t)-\mathbf{a} \varphi(t)]}{r(t) Z(t)} d t=0, \quad j=0,1, \cdots,-\varkappa-1 .
$$

Definição 1.2.10. Considere a equação

$$
a(x) \varphi(x)+\int_{\mathrm{L}} l(x, t) \varphi(t) d t=f(t)
$$

onde $a(x), l(x, t)$ e $f(x)$ são funções contínuas. Se o termo a $(x)$ é nulo, a denominamos equação de Fredholm de primeira espécie, e se $a(x) \neq 0$ equação de Fredholm de segunda espécie.

Definimos

$$
N(x, t)=\frac{a(x)}{r^{2}(x)} l(x, t)-\frac{b(x) Z(x)}{\pi r(x)} \int_{\mathrm{L}} \frac{l\left(t_{1}, t\right) d t_{1}}{Z\left(t_{1}\right) r\left(t_{1}\right)\left(t_{1}-x\right)} .
$$

Então

$$
A^{*} \mathbf{a} \varphi(x)=\int_{\mathrm{L}} N(x, t) \varphi(t) d t
$$

e a equação (1.22) pode ser escrita como:

$$
\varphi(x)+\int_{\mathrm{L}} N(x, t) \varphi(t) d t=A^{*} f-\frac{2 i b(x) Z(x)}{r(x)} P_{\varkappa-1}(x) .
$$

De (1.10), temos que

$$
Z(t)=\prod_{j=1}^{2 p}\left(t-c_{j}\right)^{\alpha_{j}} \Omega(t)
$$


para $\alpha_{j}=\gamma_{j}+i \delta_{j}+\lambda_{j}$, e $\Omega(t)$ função não nula que satisfaz a condição $\mathcal{H}(\mathrm{L})$. Suponha que os números $\alpha_{j}$ estejam ordenados de forma que

$$
\left\{\begin{aligned}
0<\boldsymbol{\operatorname { R e }}\left[\alpha_{j}\right]<1 \quad \text { se } \quad 1 \leq j \leq q \\
-1<\boldsymbol{\operatorname { R e }}\left[\alpha_{j}\right]<0 \quad \text { se } \quad q+1 \leq j \leq m \\
\boldsymbol{\operatorname { R e }}\left[\alpha_{j}\right]=0 \quad \text { se } \quad m+1 \leq j \leq 2 p
\end{aligned}\right.
$$

Se definimos

$$
T(t)=\prod_{j=q+1}^{m}\left(t-c_{j}\right)^{\alpha_{j}}
$$

então podemos escrever

$$
Z(t)=T(t) \Omega_{0}(t)
$$

onde $\Omega_{0}(t)$ é uma função que satisfaz a condição $\mathcal{H}(\mathrm{L})$.

Escreva $\varphi(t)$ sob a forma

$$
\varphi(t)=T(t) \varphi_{0}(t)
$$

Então (1.24) pode ser reescrita como

$$
\varphi_{0}(x)+\int_{L} \frac{N(x, t) T(t) \varphi_{0}(t)}{T(x)} d t=\frac{1}{T(x)}\left(A^{*} f-\frac{2 i b(x) Z(x) P_{\varkappa-1}(x)}{r(x)}\right) .
$$

Notemos que a integral em (1.28) não possui singularidade na variável $x$, mas possui na variável $t$. Para removermos esta singularidade, fazemos uma transformação de coordenadas, definindo

$$
\tau=\int_{a_{j}}^{t} T(s) d s \quad e \quad \tau_{0}=\int_{a_{j}}^{x} T(s) d s \quad \text { em } \quad \mathrm{L}_{j}=a_{j} b_{j}, 1 \leq j \leq p .
$$

Reescrevendo (1.28) nesta variável, obtemos

$$
\varphi_{0}\left(\tau_{0}\right)+\int_{\Lambda} n\left(\tau_{0}, \tau\right) \varphi_{0}(\tau) d \tau=\frac{1}{T\left(\tau_{0}\right)}\left(A^{*} f-\frac{2 i b\left(\tau_{0}\right) Z\left(\tau_{0}\right)}{r\left(\tau_{0}\right)} P_{\varkappa-1}\left(\tau_{0}\right)\right)
$$

onde $n\left(\tau_{0}, \tau\right)=N\left(\tau_{0}, \tau\right) / T\left(\tau_{0}\right)$, e $\Lambda$ denota a união dos $\operatorname{arcos} \Lambda_{j}$, correspondentes aos $\operatorname{arcos} \mathrm{L}_{j}$ do plano. Esta é uma equação de Fredholm.

Lema 1.2.11. Seja a equação de Fredholm

$$
N \varphi=a(x) \varphi(x)+\int_{\mathrm{L}} n(x, t) \varphi(t) d t=g(t)
$$


onde L é a união finita de arcos suaves não se interceptando, e a $(x)$ é uma função que não se anula em ponto algum de L. Então, se a equação

$$
N \varphi=0
$$

não possuir solução não nula, a solução de (1.30) é única, e é dada por

$$
\varphi(x)=\alpha(x) g(x)+\int_{\mathrm{L}} \varsigma(x, t) g(t) d t
$$

onde $\alpha(x)=1 / a(x)$ e $\varsigma(x, t)$ é uma função com as mesmas características de $n(x, t)$.

A demonstração deste lema, pode ser encontrada em [18, p. 137].

Deste lema, concluímos que (1.28) possui uma única solução, desde que -1 não seja autovalor do núcleo de $n\left(\tau_{0}, \tau\right)$. 


\section{Capítulo 2}

\section{Equações integro-diferenciais singulares}

Neste capítulo, propomos a resolução de um tipo de EIDS com núcleo de Cauchy generalizada. Devido ao fato de muitos problemas práticos descritos por uma EIDS ou EIS, possuírem condições de fronteira não-homogêneas, impomos estas condições na equação proposta aqui. Pelo mesmo motivo, assumimos o contorno L como sendo um arco aberto, mais especificamente, o intervalo $[-1,1]$.

Através de mudanças de variáveis, a EIDS é tranformada em uma EIS, e dessa forma podemos resolvê-la aplicando a teoria apresentada no Capítulo 1.

Apresentamos o MCP que utilizamos para resolver a EIS obtida, e a equação a ser resolvida na prática com a aplicação deste método.

\subsection{Considerações iniciais}

Considere a EIDS

$$
\begin{gathered}
a_{1} \varphi^{\prime}(x)+\frac{b_{1}}{\pi} f_{-1}^{1} \frac{\varphi^{\prime}(t)}{t-x} d t+\int_{-1}^{1} l_{1}(x, t) \varphi^{\prime}(t) d t+a_{2}(x) \varphi(x)+\int_{-1}^{1} l_{2}(x, t) \varphi(t) d t=f(x) \\
|x|<1
\end{gathered}
$$

onde $a_{2}(x)$ e $f(x)$ são funções que satisfazem a condição $\mathcal{H}^{*}([-1,1])$ e $l_{1,2}(x, t)$ satisfaz a condição $\mathcal{H}^{*}([-1,1])$ em ambas as variáveis ou $l_{1,2}(x, t)=l_{1,2}^{*}(x, t) /(x-t)$ onde $l_{1,2}^{*}(x, t)$ satisfaz a condição $\mathcal{H}([-1,1])$ em ambas as variáveis. As constantes $a_{1}, b_{1}$ devem satisfazer as condições $a_{1}^{2}+b_{1}^{2}=1$. As condições de fronteira

$$
\varphi(-1)=\xi_{1} \quad e \quad \varphi(1)=\xi_{2}
$$


também devem ser satisfeitas.

Para simplificar alguns cálculos, aplicamos a mudança de variáveis

$$
\psi(x)=\varphi(x)+c_{1} x+c_{2},
$$

com o objetivo de que

$$
\psi(-1)=0 \quad \text { e } \quad \psi(1)=0
$$

e obtemos

$$
\left\{\begin{aligned}
\psi(-1) & =\xi_{1}-c_{1}+c_{2}=0 \\
\psi(1)=\xi_{2}+c_{1}+c_{2}=0 & \Rightarrow c_{1}=\left(\xi_{1}-\xi_{2}\right) / 2 \text { e } c_{2}=-\left(\xi_{1}+\xi_{2}\right) / 2
\end{aligned}\right.
$$

donde podemos escrever

$$
\psi(x)=\varphi(x)+\frac{\left(\xi_{1}-\xi_{2}\right)}{2} x-\frac{\left(\xi_{1}+\xi_{2}\right)}{2} .
$$

Na variável $\psi(x)$, a equação (2.1) pode ser escrita na forma:

$$
\begin{gathered}
a_{1} \psi^{\prime}(x)+\frac{b_{1}}{\pi} \int_{-1}^{1} \frac{\psi^{\prime}(t)}{t-x} d t+\int_{-1}^{1} l_{1}(x, t) \psi^{\prime}(t) d t+a_{2}(x) \psi(x)+\int_{-1}^{1} l_{2}(x, t) \psi(t) d t= \\
=\bar{f}(x)
\end{gathered}
$$

onde

$$
\begin{aligned}
\bar{f}(x) & =f(x)+\frac{\left(\xi_{1}-\xi_{2}\right)}{2}\left\{a_{1}+\frac{b_{1}}{\pi} \log \left|\frac{1-x}{1+x}\right|+\int_{-1}^{1} l_{1}(x, t) d t+a_{2}(x) x+\right. \\
& \left.+\int_{-1}^{1} l_{2}(x, t) t d t\right\}-\frac{\left(\xi_{1}+\xi_{2}\right)}{2}\left[a_{2}(x)+\int_{-1}^{1} l_{2}(x, t) d t\right] .
\end{aligned}
$$

Observe que devido à mudança de variáveis aplicada, a função $\bar{f}(x)$ possui singularidades nos extremos do intervalo $[-1,1]$. A não limitação de $\bar{f}(x)$ em $[-1,1]$, gera algumas dificuldades na prova de convergência do método, que serão tratadas no próximo capítulo.

Para que possamos aplicar a teoria das EIS discutida no Capítulo 1, devemos transformar a equação (2.2) em uma equação integral. Por este motivo, fazemos outra mudança na variável dependente

$$
u(x)=\psi^{\prime}(x) \Rightarrow \psi(x)=\int_{-1}^{x} u(t) d t
$$


e substituindo em (2.2), obtemos

$$
\begin{gathered}
a_{1} u(x)+\frac{b_{1}}{\pi} \int_{-1}^{1} \frac{u(t)}{t-x} d t+\int_{-1}^{1} l_{1}(x, t) u(t) d t+a_{2}(x) \int_{-1}^{x} u(t) d t+ \\
+\int_{-1}^{1} l_{2}(x, t)\left[\int_{-1}^{t} u(s) d s\right] d t=\bar{f}(x)
\end{gathered}
$$

A condição de fronteira para $u(x)$ é $\int_{-1}^{1} u(x) d x=0$.

Fazendo uma integração por partes no termo $\int_{-1}^{1} l_{1}(x, t) u(t) d t$, a equação $(2.3)$ pode ser reescrita como:

$$
\begin{gathered}
a_{1} u(x)+\frac{b_{1}}{\pi} \int_{-1}^{1} \frac{u(t)}{t-x} d t+a_{2} \int_{-1}^{x} u(t) d t+\int_{-1}^{1}\left(-\frac{\partial l_{1}(x, t)}{\partial t}+l_{2}(x, t)\right)\left(\int_{-1}^{t} u(s) d s\right) d t= \\
\bar{f}(x),
\end{gathered}
$$

$\operatorname{com} \int_{-1}^{1} u(t) d t=0$.

A partir de (1.5), (1.6) e (1.16), obtemos

$$
\gamma_{j}+i \delta_{j}=\mp \frac{1}{2 \pi i} \ln \left[\frac{a_{1}-i b_{1}}{a_{1}+i b_{1}}\right], \quad j=1,2,
$$

sendo o sinal acima negativo para $j=1$, ou seja, para o extremo $c_{1}=-1$, e positivo para $j=2$, para o extremo $c_{2}=1$.

De acordo com a teoria do Capítulo 1, devemos encontrar $M$ e $N$ inteiros que satisfaçam as desigualdades

$$
-1<-\gamma_{1}+M<1 \quad \text { e } \quad-1<\gamma_{1}+N<1
$$

e por (1.25),

$$
Z(x)=(1-x)^{-\gamma_{1}-i \delta_{1}+M}(1+x)^{\gamma_{1}+i \delta_{1}+N} \Omega(x)=(1-x)^{\alpha}(1+x)^{\beta} \Omega^{*}(x),
$$

para $\alpha$ e $\beta$ dados por

$$
\alpha=-\gamma_{1}+M \text { e } \beta=\gamma_{1}+N
$$

Portanto, o índice do problema é

$$
\varkappa=-(M+N)=-(\alpha+\beta)
$$

Pelas condições sobre $M$ e $N$ dadas em $(2.5)$, o valor de $\varkappa$ se restringe a $\{-1,0,1\}$. No caso em que $\varkappa=1$, a solução de (2.4) é de classe $h_{0}$ e é ilimitada nos extremos -1 e 
1, além de $-1<\alpha, \beta<0$. Devido à forma da equação (2.4) e sua condição de fronteira, devemos procurar soluções que pertençam a esta classe.

Das equações (1.26) e (1.27), podemos escrever

$$
\varphi(x)=Z(x) g(x)
$$

onde $Z(x)$ é função fundamental da classe $h_{0}$ do problema de Riemann-Hilbert associado à equação $(2.4)$, e $g(x)$ satisfaz a condição $\mathcal{H}^{*}([-1,1])$.

Denotaremos a função $Z(x)$ por $\omega^{\alpha, \beta}(x)$. Esta será a função peso da equação de interesse. Disto, temos que (2.4) pode ser reescrita como:

$$
\begin{aligned}
& a_{1} \omega^{\alpha, \beta}(x) g(x)+\frac{b_{1}}{\pi} f_{-1}^{1} \frac{\omega^{\alpha, \beta}(t) g(t)}{t-x} d t+a_{2}(x) \int_{-1}^{x} \omega^{\alpha, \beta}(t) g(t) d t+ \\
& +\int_{-1}^{1}\left(-\frac{\partial l_{1}(x, t)}{\partial t}+l_{2}(x, t)\right)\left(\int_{-1}^{t} \omega^{\alpha, \beta}(s) g(s) d s\right) d t=\bar{f}(x),
\end{aligned}
$$

$\operatorname{com} \int_{-1}^{1} \omega^{\alpha, \beta}(t) g(t) d t=0$.

Definimos os operadores

$$
\left\{\begin{array}{l}
H g(x)=a_{1} \omega^{\alpha, \beta}(x) g(x)+\frac{b_{1}}{\pi} \int_{-1}^{1} \frac{\omega^{\alpha, \beta}(t) g(t)}{t-x} d t \\
D f(x)=a_{2}(x) f(x) \\
R g(x)=\int_{-1}^{x} \omega^{\alpha, \beta}(t) g(t) d t \\
l(x, t)=-\frac{\partial l_{1}(x, t)}{\partial t}+l_{2}(x, t) \\
L f(x)=\int_{-1}^{1} l(x, t) f(t) d t \\
\widetilde{H} g(x)=a_{1} \omega^{-\alpha,-\beta}(x) g(x)-\frac{b_{1}}{\pi} \int_{-1}^{1} \frac{g(t) \omega^{-\alpha,-\beta}(t)}{t-x} d t
\end{array}\right.
$$

Então, a equação (2.6) escrita em termos de operadores toma a forma:

$$
[H+(D+L) R] g=\bar{f}
$$

Utilizando o Teorema 1.2.9, em [6] mostrou-se que o operador $\widetilde{H}$ satisfaz

$$
\widetilde{H} H g= \begin{cases}g+g_{0} & \text { se } \varkappa=1, \\ g & \text { se } \varkappa \leq 0, \text { e para } 0 \leq j \leq-\varkappa-1, \\ & \int_{-1}^{1} \frac{x^{j}}{Z(x)}\left[a_{1} \omega^{\alpha, \beta}(x) g(x)+\frac{b_{1}}{\pi} \int_{-1}^{1} \frac{\omega^{\alpha, \beta}(t) g(t)}{t-x} d t\right] d x=0,\end{cases}
$$


para $g_{0} \in$ ker $H$, uma constante arbitrária. Assim,

$$
\begin{cases}\{I+\widetilde{H}[(D+L) R]\} g=\widetilde{H} f-g_{0}, & \text { se } \varkappa=1, \\ \{I+\widetilde{H}[(D+L) R]\} g=\widetilde{H} f, & \text { se } \varkappa \leq 0,\end{cases}
$$

e então, se $\varkappa \leq 0, \widetilde{H}$ é inversa à esquerda de $H, \forall g \in \mathcal{H}^{*}([-1,1])$ e $\int_{-1}^{1} \omega^{\alpha, \beta}(t) g(t) d t=0$.

Como visto no Capítulo 1, as equações (2.8) são equações de Fredholm de segunda espécie, e possuem solução única, desde que -1 não seja autovalor de $\widetilde{H}[(D+L) R]$ caso $\varkappa \leq 0$. No caso de $\varkappa=1$, devemos determinar a constante $g_{0}$ para obtermos uma única solução, impondo em $(2.8)$ a condição $\int_{-1}^{1} \omega^{\alpha, \beta}(t) g(t) d t=0$. Se o operador $[I+\widetilde{H}[(D+L) R]$ for inversível, multiplicando a primeira equação de (2.8) por $\left[I+\widetilde{H}[(D+L) R]^{-1}\right.$, e em seguida por $\omega^{\alpha, \beta}(x)$, integrando a equação resultante sobre $[-1,1]$ em $x$, obtemos

$0=\int_{-1}^{1} \omega^{\alpha, \beta}(x)\left\{[I+\widetilde{H}[(D+L) R]]^{-1} \widetilde{H} f\right\}(x) d x-\int_{-1}^{1} \omega^{\alpha, \beta}(x)\{I+\widetilde{H}[(D+L) R]\}^{-1} g_{0} d x$.

Então para determinar $g_{0}$ de maneira única, devemos ter:

$$
\int_{-1}^{1} \omega^{\alpha, \beta}(x)\{I+\widetilde{H}[(D+L) R]\}^{-1} 1 d x \neq 0 .
$$

Vamos considerar que esta condição é satisfeita.

\subsection{Método de colocação polinomial}

Primeiramente, relembremos alguns resultados clássicos relacionados aos polinômios ortogonais.

Definição 2.2.1. São chamados polinômios de Jacobi de grau n, os polinômios $P_{n}^{\alpha, \beta}(t)$ tais que

$$
\int_{-1}^{1} \omega^{\alpha, \beta}(t) P_{i}^{\alpha, \beta}(t) P_{j}^{\alpha, \beta}(t) d t=h_{i} \delta_{i j}, \quad i, j=0,1, \ldots
$$

para $\alpha, \beta>-1, \omega^{\alpha, \beta}(t)$ conforme definido anteriormente, $\delta_{i, j}$ o delta de Kronecker $e$

$$
h_{i}=\frac{2^{\alpha+\beta+1}}{2 i+\alpha+\beta+1} \frac{\Gamma(i+\alpha+1) \Gamma(i+\beta+1)}{\Gamma(i+1) \Gamma(i+\alpha+\beta+1)} .
$$


Definimos a aproximação da função $g(x)$, como sendo:

$$
g_{n}(x)=c_{0} P_{0}^{\alpha, \beta}(x)+c_{1} P_{1}^{\alpha, \beta}(x)+\cdots+c_{n} P_{n}^{\alpha, \beta}(x),
$$

onde

$c_{j}=\frac{2^{\alpha+\beta+1}}{2 j+\alpha+\beta+1} \frac{\Gamma(j+\alpha+1) \Gamma(j+\beta+1)}{\Gamma(j+1) \Gamma(j+\alpha+\beta+1)} \int_{-1}^{1} \omega^{\alpha, \beta}(x) P_{j}^{\alpha, \beta}(x) g(x) d x, \quad 0 \leq j \leq n$, (veja [22], equação (9.1.1)). Observe que devido às condições de fronteira impostas em $(2.6), c_{0}=0$.

Substituindo $g_{n}$ em (2.6), podemos definir o resíduo

$$
\begin{aligned}
& r_{n}(x)=\sum_{j=1}^{n} c_{j}\left\{a_{1} \omega^{\alpha, \beta}(x) P_{j}^{\alpha, \beta}(x)+\frac{b_{1}}{\pi} \int_{-1}^{1} \frac{\omega^{\alpha, \beta}(t) P_{j}^{\alpha, \beta}(t)}{(t-x)} d t+\right. \\
& \left.+a_{2}(x) \int_{-1}^{x} \omega^{\alpha, \beta}(t) P_{j}^{\alpha, \beta}(t) d t+\int_{-1}^{1} l(x, t)\left[\int_{-1}^{t} \omega^{\alpha, \beta}(s) P_{j}^{\alpha, \beta}(s) d s\right] d t\right\}-\bar{f}(x) .
\end{aligned}
$$

Da fórmula de Rodrigues generalizada (veja [22], equação (4.10.1)), temos que

$$
\omega^{\alpha, \beta}(x) P_{j}^{\alpha, \beta}(x)=-\frac{1}{2 j} \frac{d}{d x}\left[\omega^{\alpha+1, \beta+1} P_{j-1}^{\alpha+1, \beta+1}(x)\right],
$$

o que implica em

$$
\int_{-1}^{x} \omega^{\alpha, \beta}(t) P_{j}^{\alpha, \beta}(t) d t=-\frac{1}{2 j} \omega^{\alpha+1, \beta+1}(x) P_{j-1}^{\alpha+1, \beta+1}(x) .
$$

Aplicando este resultado em (2.11), obtemos

$$
\begin{aligned}
& r_{n}(x)=\sum_{j=1}^{n} c_{j}\left\{a_{1} \omega^{\alpha, \beta}(x) P_{j}^{\alpha, \beta}(x)+\frac{b_{1}}{\pi} \int_{-1}^{1} \frac{\omega^{\alpha, \beta}(t) P_{j}^{\alpha, \beta}(t)}{(t-x)} d t-\right. \\
& \left.-\frac{1}{2 j}\left[a_{2}(x) \omega^{\alpha+1, \beta+1}(x) P_{j-1}^{\alpha+1, \beta+1}(x)+\int_{-1}^{1} l(x, t) \omega^{\alpha+1, \beta+1}(t) P_{j-1}^{\alpha+1, \beta+1}(t) d t\right]\right\}- \\
& -\bar{f}(x) .
\end{aligned}
$$

Para tratar os dois primeiros termos de (2.14), utilizaremos o próximo lema.

Lema 2.2.2. ([6], Lema 3.4.1) Sejam $\left\{P_{n}^{\alpha, \beta}\right\}$ e $\left\{P_{n}^{-\alpha,-\beta}\right\}$ sequências de polinômios de Jacobi de grau n, ortogonais com respeito aos pesos $\omega^{\alpha, \beta}(x)$ e $\omega^{-\alpha,-\beta}(x)$ respectivamente, $\operatorname{com} \alpha, \beta$ não inteiros. Então,

$$
\begin{aligned}
& a \omega^{\alpha, \beta}(x) P_{n}^{\alpha, \beta}(x)+\frac{b}{\pi} \int_{-1}^{1} \frac{\omega^{\alpha, \beta}(t) P_{n}^{\alpha, \beta}(t)}{(t-x)} d t=-\frac{2^{-\varkappa}}{\operatorname{sen}(\pi \alpha)} b P_{n-\varkappa}^{-\alpha,-\beta}(x), \\
& a \omega^{-\alpha,-\beta}(x) P_{n}^{-\alpha,-\beta}(x)-\frac{b}{\pi} \int_{-1}^{1} \frac{\omega^{-\alpha,-\beta}(t) P_{n}^{-\alpha,-\beta}(t)}{(t-x)} d t=-\frac{2^{\varkappa}}{\operatorname{sen}(\pi \alpha)} b P_{n+\varkappa}^{\alpha, \beta}(x),
\end{aligned}
$$

onde $P_{n}^{\alpha, \beta}=P_{n}^{-\alpha,-\beta} \equiv 0$ se $n<0$ e $\varkappa$ é o indice do problema. 
Aplicando o Lema 2.2.2 na equação (2.14), obtemos

$$
\begin{aligned}
r_{n}(x) & =\sum_{j=1}^{n} c_{j}\left\{\frac{-b_{1} P_{j-1}^{-\alpha,-\beta}(x)}{2 \operatorname{sen}(\pi \alpha)}-\frac{1}{2 j}\left[a_{2}(x) \omega^{\alpha+1, \beta+1}(x) P_{j-1}^{\alpha+1, \beta+1}(x)+\right.\right. \\
& \left.\left.+\int_{-1}^{1} l(x, t) \omega^{\alpha+1, \beta+1}(t) P_{j-1}^{\alpha+1, \beta+1}(t) d t\right]\right\}-\bar{f}(x) .
\end{aligned}
$$

O resíduo $r_{n}(x)$ pode ser escrito na forma:

$$
r_{n}(x)=\left\{[H+(D+L) R] g_{n}-\bar{f}\right\}(x) .
$$

Definição 2.2.3. O método de colocação polinomial consiste na aproximação da fun$c ̧ \tilde{a} o g(x)$ por $g_{n}(x)=\sum_{j=0}^{n} c_{j} P_{j}(x)$, onde $\left\{P_{j}\right\}_{j=1}^{n}$ é uma base para o conjunto de polinômios de grau $n$, e os coeficientes $c_{j}$ são determinados a partir da equação residual, quando a ela é imposta a condição $r_{n}\left(x_{i}\right)=0$ para $n$ pontos distintos $x_{i}$, denominados pontos de colocação, que são escolhidos de forma conveniente sobre $[-1,1]$.

Impondo que $r_{n}\left(x_{i}\right)=0$ para $0 \leq i \leq n$, obtemos sistema de equações lineares

$$
\begin{aligned}
r_{n}\left(x_{i}\right) & =\sum_{j=1}^{n} c_{j}\left\{\frac{-b_{1} P_{j-1}^{-\alpha,-\beta}\left(x_{i}\right)}{2 \operatorname{sen}(\pi \alpha)}-\frac{1}{2 j}\left[a_{2}\left(x_{i}\right) \omega^{\alpha+1, \beta+1}\left(x_{i}\right) P_{j-1}^{\alpha+1, \beta+1}\left(x_{i}\right)+\right.\right. \\
& \left.\left.+\int_{-1}^{1} l\left(x_{i}, t\right) \omega^{\alpha+1, \beta+1}(t) P_{j-1}^{\alpha+1, \beta+1}(t) d t\right]\right\}-\bar{f}\left(x_{i}\right)=0 .
\end{aligned}
$$

Escolhemos como pontos de colocação, os zeros do polinômio de Chebyshev de primeira espécie de grau $n$

$$
x_{i}=\cos \left[\frac{(2 i+1) \pi}{2 n}\right], \quad 1 \leq i \leq n .
$$

O motivo desta escolha ficará claro mais adiante, na Seção 3.3.

Observe que a integral

$$
\int_{-1}^{1} l(x, t) \omega^{\alpha+1, \beta+1}(t) P_{j-1}^{\alpha+1, \beta+1}(t) d t
$$

em geral não pode ser resolvida analiticamente. Por este motivo, quando necessário, empregamos neste termo uma quadratura de Gauss-Jacobi com $n$ nós, dada por

$$
\begin{aligned}
\int_{-1}^{1} l(x, t) \omega^{\alpha+1, \beta+1}(t) P_{j-1}^{\alpha+1, \beta+1}(t) d t & \simeq \sum_{i=1}^{n} l\left(x, t_{i}\right) P_{j-1}^{\alpha+1, \beta+1}\left(t_{i}\right) \lambda_{i}^{\alpha+1, \beta+1}:= \\
& :=\widetilde{L}^{n}\left[P_{j-1}^{\alpha+1, \beta+1}(x) \omega^{\alpha+1, \beta+1}(x)\right]
\end{aligned}
$$


onde

$$
\lambda_{i}^{\alpha+1, \beta+1}=2^{\alpha+\beta+3} \frac{\Gamma(n+\alpha+2) \Gamma(n+\beta+2)}{\Gamma(n+1) \Gamma(n+\alpha+\beta+3)}\left(1-t_{i}^{2}\right)^{-1}\left[\left(P_{n}^{\alpha+1, \beta+1}\right)^{\prime}\left(t_{i}\right)\right]^{-2},
$$

(veja [22], equação (15.3.1) ), e $t_{i}, 1 \leq i \leq n$, denotam as $n$ raízes do polinômio $P_{n}^{\alpha+1, \beta+1}(t)$, obtidas com o auxílio do software MATHEMATICA.

De [22], equação (4.21.7), obtemos a identidade

$$
\frac{d}{d x}\left\{P_{n}^{\alpha, \beta}(x)\right\}=\frac{1}{2}(n+\alpha+\beta+1) P_{n-1}^{\alpha+1, \beta+1}(x),
$$

que aplicada em (2.18), nos leva à identidade

$$
\begin{aligned}
\widetilde{L}^{n}\left[P_{j-1}^{\alpha+1, \beta+1}(x) \omega^{\alpha+1, \beta+1}(x)\right]= & \frac{2^{\alpha+\beta+5}}{(n+\alpha+\beta+3)^{2}} \frac{\Gamma(n+\alpha+2) \Gamma(n+\beta+2)}{\Gamma(n+1) \Gamma(n+\alpha+\beta+3)} . \\
& \cdot \sum_{i=1}^{n} l\left(x, t_{i}\right) P_{j-1}^{\alpha+1, \beta+1}\left(t_{i}\right)\left(1-t_{i}^{2}\right)^{-1}\left[P_{n-1}^{\alpha+2, \beta+2}\left(t_{i}\right)\right]^{-2} .
\end{aligned}
$$

Para calcularmos os polinômios $P_{j}^{-\alpha,-\beta}(x), P_{j}^{\alpha+1, \beta+1}(x), 1 \leq j \leq n-1$ e $P_{n-1}^{\alpha+2, \beta+2}(x)$, lançamos mão da identidade

$$
P_{n}^{\alpha, \beta}(x)=\sum_{k=0}^{n}\left(\begin{array}{c}
n+\alpha \\
n-k
\end{array}\right)\left(\begin{array}{c}
n+\beta \\
k
\end{array}\right)\left(\frac{x-1}{2}\right)^{k}\left(\frac{x+1}{2}\right)^{n-k},
$$

(veja [22], equação (4.3.2)). Nos casos particulares em que $\alpha=\beta=-1 / 2$ e $\alpha=\beta=1 / 2$, as expressões dos polinômios possuem uma forma mais simplificada que a anterior, dadas por:

$$
P_{n}^{-1 / 2,-1 / 2}(x)=\frac{1}{4^{n}}\left(\begin{array}{c}
2 n \\
n
\end{array}\right) T_{n}(x) \text { e } P_{n}^{1 / 2,1 / 2}(x)=\frac{2}{\sqrt{\pi}} \frac{\Gamma(n+3 / 2)}{\Gamma(n+2)} U_{n}(x),
$$

onde $T_{n}(x)=\cos (n \arccos x)$ e $U_{n}(x)=\operatorname{sen}[(n+1) \arccos x] / \operatorname{sen}(\arccos x)$ são os polinômios de Chebyshev de primeira e segunda espécie, respectivamente (veja [1], equações $(22.5 .23)$ e $(22.5 .32))$.

O sistema de equações lineares que resolvemos na prática é $A c=\bar{f}$, onde

$$
\begin{aligned}
& A_{i, j}= \frac{-b_{1} P_{j-1}^{-\alpha,-\beta}\left(x_{i}\right)}{2 \operatorname{sen}(\pi \alpha)}-\frac{1}{2 j}\left[a_{2}\left(x_{i}\right) \omega^{\alpha+1, \beta+1}\left(x_{i}\right) P_{j-1}^{\alpha+1, \beta+1}\left(x_{i}\right)+\right. \\
&\left.+\sum_{k=1}^{n} l\left(x_{i}, t_{k}\right) P_{j-1}^{\alpha+1, \beta+1}\left(t_{k}\right) \lambda_{k}^{\alpha+1, \beta+1}\right], 1 \leq i, j \leq n, \\
& c=\left[c_{1} \cdots c_{n}\right]^{\top} \text { e } \bar{f}=\left[\bar{f}\left(x_{1}\right) \cdots \bar{f}\left(x_{n}\right)\right]^{\top} .
\end{aligned}
$$




\section{Capítulo 3}

\section{Espaço de Operadores}

Neste capítulo, apresentamos as propriedades de mapeamento dos operadores definidos em (2.7), quando aplicados em espaços ponderados de Besov.

Primeiramente, apresentamos os principais conceitos relativos aos espaços ponderados de funções contínuas e de subspaços destes, do tipo Besov, aos quais consideramos pertencer os coeficientes da equação (2.6). Em seguida, mostramos como se comportam os operadores de (2.7) nestes espaços, principalmente no que tange à sua limitação. Finalmente, apresentamos uma análise de convergência do MCP, segundo a norma uniforme ponderada.

\subsection{Conceitos e teoremas principais}

Definição 3.1.1. Para $\rho, \tau \geq 0$, definimos o espaço de funções

$$
C_{\rho, \tau}:=\left\{f \in C(-1,1): f \omega^{\rho, \tau} \in C[-1,1]\right\}
$$

equipado da norma

$$
\|f\|_{\infty, \rho, \tau}=\max _{|x| \leq 1}\left|\left(f \omega^{\rho, \tau}\right)(x)\right| .
$$

Notação. Denotamos por $C_{\rho, \tau}^{0}$, o subspaço de $C_{\rho, \tau}$ das funções $f$ tais que

$$
\left\{\begin{array}{lll}
\left(f \omega^{\rho, \tau}\right)(1)=0 & \text { se } & \rho>0 \\
\left(f \omega^{\rho, \tau}\right)(-1)=0 & \text { se } & \tau>0 .
\end{array}\right.
$$

Notação. Denotamos por $\Pi_{n}$, o conjunto dos polinômios de grau no máximo n. 
Notação. Denotamos por $E_{n}^{\rho, \tau}(f)$, o erro da melhor aproximação uniforme ponderada de $f$ por polinômios em $\Pi_{n}$, isto é,

$$
E_{n}^{\rho, \tau}(f)=\inf \left\{\left\|f-p_{n}\right\|_{\infty, \rho, \tau}: p_{n} \in \Pi_{n}\right\}
$$

Definição 3.1.2. Seja $\mathscr{B}=\left\{b_{n}\right\}$ uma sequência de números reais positivos, tal que $\lim _{n \rightarrow \infty} b_{n}=0$. Denotamos por $C_{\rho, \tau}^{\mathscr{B}}$, o espaço ponderado de Besov

$$
C_{\rho, \tau}^{\mathscr{B}}:=\left\{f \in C_{\rho, \tau}:\|f\|_{\infty, \rho, \tau}+\sup _{n=1,2, \ldots} \frac{E_{n}^{\rho, \tau}(f)}{b_{n}}<\infty\right\}
$$

Proposição 3.1.3. ([12], Proposição 3.1) $C_{\rho, \tau}^{\mathscr{B}}$ é espaço de Banach.

Lema 3.1.4. ([12], Lema 3.2) Se $\mathscr{B}=\left\{b_{n}\right\}$ e $\mathscr{C}=\left\{c_{n}\right\}$ são sequências de números reais positivos, tais que $\lim _{n \rightarrow \infty}\left(b_{n} / c_{n}\right)=0$, então $C_{\rho, \tau}^{\mathscr{B}}$ é compactamente imerso em $C_{\rho, \tau}^{\mathscr{C}}$.

Observação 3.1.5. ([12], Observação 3.4) Se existir uma constante positiva $M$, tal que $b_{n} \leq M c_{n}$, a imersão $C_{\rho, \tau}^{\mathscr{B}} \subset C_{\rho, \tau}^{\mathscr{C}}$ é contínua.

Observação 3.1.6. ([12], Observação 3.5) $S e \rho_{1} \leq \rho_{2}$ e $\tau_{1} \leq \tau_{2}$, a imersão $C_{\rho_{1}, \tau_{1}}^{\mathscr{B}} \subset C_{\rho_{2}, \tau_{2}}^{\mathscr{C}}$ é contínua.

Definição 3.1.7. Sejam $p$ um inteiro não negativo e $\theta \in(0,1]$. Denotamos por $C^{p, \theta}$, o espaço de Banach das funções a valores reais de classe $C^{p}[-1,1]$, tais que sua p-ésima derivada satisfaz a condição $\mathcal{H}^{\theta}([-1,1])$. A norma em $C^{p, \theta}$ é dada por:

$$
\|u\|_{p, \theta}:=\sum_{k=0}^{p}\left\|u^{(k)}\right\|_{\infty}+\sup \left\{\frac{|u(x)-u(t)|}{|x-t|^{\theta}}: x, t \in[-1,1], x \neq t\right\} .
$$

Notação. Denotamos por

$\rho_{0}=\left\{\begin{array}{ll}\min \{\rho, 1\} & \text { se } \rho>0, \\ 1 & \text { se } \rho=0 .\end{array} \quad\right.$ e $\quad \tau_{0}=\left\{\begin{array}{lll}\min \{\tau, 1\} & \text { se } & \tau>0 \\ 1 & \text { se } & \tau=0\end{array}\right.$

Notação. Suponha que existam constantes positivas $M$ e $\gamma$, tais que $b_{n} \leq M n^{-\gamma}$, $n=1,2, \ldots$ Então, dizemos que $b_{n}=O\left(n^{-\gamma}\right)$. 
Lema 3.1.8. ([12], Lema 3.11) Suponha que $\mathscr{B}=\left\{b_{n}\right\}$, seja tal que $b_{n}=O\left(n^{-\gamma}\right)$, e que $f \in C_{\rho, \tau}^{\mathscr{B}}$. Então, exitem constantes positivas $\nu=\min \left\{\rho_{0}, \tau_{0}\right\} /[\gamma+2(1+\max \{\rho, \tau\})]$ e c, tais que

$$
f \omega^{\rho, \tau} \in C^{0, \nu \gamma} \quad e \quad\left\|f \omega^{\rho, \tau}\right\|_{0, \nu \gamma} \leq c\|f\|_{\rho, \tau, \mathscr{B}}
$$

A exigência de que as funções $a_{2}(x), l(x, t)$ e $\bar{f}(x) \in C_{\rho, \tau}^{\mathscr{B}}$ para certos valores de $\rho, \tau$ e algum $\mathscr{B}$, será imposta nos lemas e teoremas a seguir. Segundo o Lema 3.1.8, isto garante que os termos da equação (2.6) satisfaçam a condição $\mathcal{H}^{*}([-1,1])$, conforme a teoria do Capítulo 1 exige para ser aplicada.

\subsection{Propriedades de mapeamento}

Primeiramente apresentamos alguns resultados que serão utilizados na demonstração de convergência do MCP.

Definição 3.2.1. Definimos as constantes não negativas $\alpha^{+}, \alpha^{-}, \beta^{+}$e $\beta^{-}$por:

$$
\alpha=\alpha^{+}-\alpha^{-} \quad \text { e } \beta=\beta^{+}-\beta^{-}, \quad 0 \leq \alpha^{ \pm}, \beta^{ \pm}<1
$$

Proposição 3.2.2. ([12], Proposição 4.4) Seja $f \in C_{\alpha^{-}, \beta^{-}}^{\mathscr{B}}$ Então $\widetilde{H} f \in C_{\alpha^{+}, \beta^{+}} e$

$$
\|\widetilde{H} f\|_{\infty, \alpha^{+}, \beta^{+}} \leq c\left(\frac{\|f\|_{\alpha^{-}, \beta^{-}, \mathscr{B}}}{n^{\mu}}+\|f\|_{\infty, \alpha^{-}, \beta^{-}} \log n\right)
$$

onde $f \omega^{\alpha^{-}, \beta^{-}} \in \mathcal{H}^{\mu}([-1,1])$.

Corolário 3.2.3. ([12], Corolário 4.5) Para $P_{n} \in \Pi_{n}, n \geq 2$,

$$
\left\|\widetilde{H} P_{n}\right\|_{\infty, \alpha^{+}, \beta^{+}} \leq c\left\|P_{n}\right\|_{\infty, \alpha^{-}, \beta^{-}} \log n
$$

Notação. Se $X$ e $Y$ são espaços de Banach, denotaremos por $\mathcal{L}(X, Y)$ o espaço de todos os operadores lineares limitados de $X$ em $Y$.

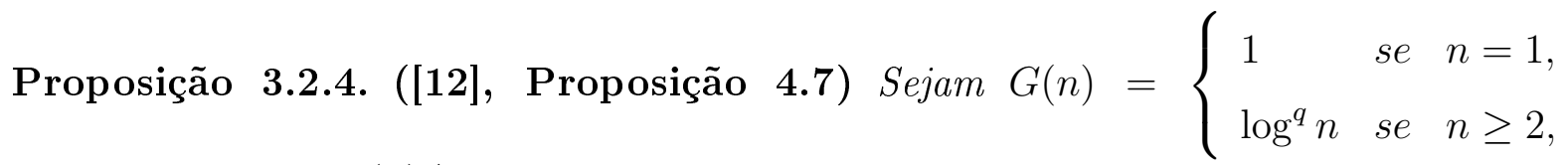
para $q \geq 0$ e $b_{n}=G(n) / n^{\gamma}$ para $\gamma>0$. Então, para $n$ suficientemente grande, temos

$$
\widetilde{H} \in \mathcal{L}\left(C_{\alpha^{-}, \beta^{-}}^{\mathscr{B}}, C_{\alpha^{+}, \beta^{+}}^{\mathscr{B} \log n}\right) \quad e \quad H \in \mathcal{L}\left(C_{\alpha^{+}, \beta^{+}}^{\mathscr{B}}, C_{\alpha^{-}, \beta^{-}}^{\mathscr{B} \log n}\right)
$$


Notação. Dizemos que $l(x, t) \in C_{\rho, \tau, x}^{\mathscr{B}} \cap C_{\nu, \varsigma, t}$, quando

i) $l(x, t) \omega^{\rho, \tau}(x) \omega^{\nu, \varsigma}(t) \in C[-1,1]^{2} e$

ii) $l_{t}^{\nu, \varsigma}=l(x, t) \omega^{\nu, \varsigma}(t) \in C_{\rho, \tau}^{\mathscr{B}}$ uniformemente com respeito a $t \in[-1,1]$.

O lema seguinte será de grande utilidade nas demonstrações das próximas proposições.

Lema 3.2.5. ([12], Lema 4.11) Seja $l(x, t) \in C_{\rho, \tau, x}^{\mathscr{B}} \cap C_{\nu, \varsigma, t}$. Então existe uma sequência $\left\{P_{n}\right\}_{n=1}^{\infty}$ de polinômios $P_{n}(x, t)=\sum_{j=0}^{n} c_{n j}(t) x^{j}$ de grau nãa maior que $n$ em $x$, onde $c_{n j}(t) \omega^{\nu, \varsigma}(t)$ é constante por partes para $j=0, \cdots, n e$

$$
\sup _{x, t \in[-1,1]}\left|\left[l(x, t)-P_{n}(x, t)\right] \omega^{\rho, \tau}(x) \omega^{\nu, \varsigma}(t)\right| \leq c b_{n}, \quad n=1,2, \ldots,
$$

onde c não depende de $n$.

As duas proposições seguintes são adaptações das Proposições 4.12 e 4.13 de [12], que se fizeram necessárias devido à mudança no domínio do operador $L$.

Proposição 3.2.6. Sejam $\nu, \varsigma$ constantes não negativas, tais que $\nu+\alpha^{-}, \varsigma+\beta^{-}<1$. Se $l(x, t) \in C_{\rho, \tau, x}^{\mathscr{B}} \cap C_{\nu, \varsigma, t}$, então $L \in \mathcal{L}\left(C_{\alpha^{-}, \beta^{-}}, C_{\rho, \tau}^{\mathscr{B}}\right)$.

Demonstração: Sejam $l(x, t) \in C_{\rho, \tau, x}^{\mathscr{B}} \cap C_{\nu, \varsigma, t}$ e $f \in C_{\alpha^{-}, \beta^{-}}$, temos:

$$
\begin{aligned}
& \left|(L f)(x) \omega^{\rho, \tau}(x)\right|=\left|\int_{-1}^{1} l(x, t) f(t) \omega^{\rho, \tau}(x) d t\right| \leq \int_{-1}^{1}\left|l(x, t) f(t) \omega^{\rho, \tau}(x) \omega^{\nu, \varsigma}(t) \omega^{-\nu,-\varsigma}(t)\right| d t \leq \\
& \leq c \int_{-1}^{1}\left|\omega^{-\nu+\alpha^{-}-\alpha^{-},-\varsigma+\beta^{-}-\beta^{-}}(t) f(t)\right| d t \leq c\|f\|_{\infty, \alpha^{-}, \beta^{-}} \int_{-1}^{1} \omega^{-\nu-\alpha^{-},-\varsigma-\beta^{-}}(t) d t .
\end{aligned}
$$

Como $\nu+\alpha^{-}, \varsigma+\beta^{-}<1$, a integral $\int_{-1}^{1} \omega^{-\nu-\alpha^{-},-\varsigma-\beta^{-}}(t) d t$ é limitada. Portanto,

$$
\|L f\|_{\infty, \rho, \tau} \leq c\|f\|_{\infty, \alpha^{-}, \beta^{-}}
$$

Definimos

$$
Q_{n}(x)=\int_{-1}^{1} P_{n}(x, t) f(t) d t
$$

onde $P_{n}(x, t)$ são dados pelo Lema 3.2.5. Portanto $Q_{n} \in \Pi_{n}$ se $c_{n j}(t) \omega^{\nu, \varsigma}(t) \in L^{\infty}(-1,1)$. 
Então obtemos:

$$
\begin{aligned}
& \left|\left(L f-Q_{n}\right)(x) \omega^{\rho, \tau}(x)\right|=\left|\int_{-1}^{1}\left[l(x, t)-P_{n}(x, t)\right] f(t) \omega^{\rho, \tau}(x) d t\right| \leq \\
& \leq \int_{-1}^{1}\left|\left[l(x, t)-P_{n}(x, t)\right] f(t)\right| \omega^{\rho, \tau}(x) \omega^{\nu-\nu, \varsigma-\varsigma}(t) d t \leq \\
& \leq c b_{n} \int_{-1}^{1}|f(t)| \omega^{-\nu+\alpha^{-}-\alpha^{-},-\varsigma+\beta^{-}-\beta^{-}}(t) d t \leq \\
& \leq c b_{n}\|f\|_{\infty, \alpha^{-}, \beta^{-}} \int_{-1}^{1} \omega^{-\nu-\alpha^{-},-\varsigma-\beta^{-}}(t) d t \leq c b_{n}\|f\|_{\infty, \alpha^{-}, \beta^{-}} .
\end{aligned}
$$

Portanto,

$$
E_{n}^{\rho, \tau}(L f) \leq c b_{n}\|f\|_{\infty, \alpha^{-}, \beta^{-}} \quad e \quad L f \in C_{\rho, \tau}^{\mathscr{B}} .
$$

Proposição 3.2.7. Seja $l(x, t)=[k(x, t)-k(t, t)] /(t-x) \operatorname{com} k(x, t) \in C_{0,0, x}^{\mathscr{B}} \cap C_{0,0, t}$. Então,

$$
L \in \mathcal{L}\left(C_{\alpha^{-}, \beta^{-}}, C_{\alpha^{-}, \beta^{-}}^{\mathscr{B} \log n}\right), \quad n \geq 2
$$

Demonstração: Pelo Lema 3.1.8, segue que $k_{t}^{0,0} \in \mathcal{H}^{\mu}([-1,1])$ uniformemente com respeito a $t \in[-1,1]$, para algum $0<\mu<1$. Usando o fato que

$$
\int_{-1}^{1}|t-x|^{\mu-1} \omega^{-\rho^{+},-\tau^{+}}(t) d t \leq c \omega^{-\rho^{+},-\tau^{+}}(x),
$$

de [3], e supondo que $f \in C_{\alpha^{-}, \beta^{-}}$, obtemos:

$$
\begin{aligned}
|L f(x)| & =\left|\int_{-1}^{1} \frac{k(x, t)-k(t, t)}{t-x} f(t) d t\right| \leq c\|f\|_{\infty, \alpha^{-}, \beta^{-}} \int_{-1}^{1}|t-x|^{\mu-1} \omega^{-\alpha^{-},-\beta^{-}}(t) d t \leq \\
& \leq c\|f\|_{\infty, \alpha^{-}, \beta^{-}} \omega^{-\alpha^{-},-\beta^{-}}(x),
\end{aligned}
$$

ou seja, $L f \in C_{\alpha^{-}, \beta^{-}}$.

Seja $P_{n}(x, t)$ a sequência de polinômios do Lema 3.2.5 para a função $k(x, t)$. Como $P_{n}(x, t)=\sum_{j=0}^{n}\left(\frac{\partial^{j}}{\partial x^{j}} P_{n}(x, t)\right)(t) \frac{(x-t)^{j}}{j !} \Longrightarrow \frac{P_{n}(x, t)-P_{n}(t, t)}{t-x}=\sum_{j=0}^{n-1} d_{n j}(t) x^{j}$ $\operatorname{com} d_{n j} \in L^{\infty}(-1,1)$, então

$$
Q_{n}(x)=\frac{1}{\pi} \int_{-1}^{1} \frac{P_{n}(x, t)-P_{n}(t, t)}{t-x} f(t) d t \in \Pi_{n}
$$


Seja $\mathscr{B}=\left\{b_{n}\right\}$ como definido na Proposição 3.2.4. Neste caso, existe um $\delta>0$ para o qual $n^{-\delta}=O\left(b_{n}\right)$. Escolha algum $m \geq(2+\delta) / 2 \mu$. Então, aplicando o Lema 3.2.5 e a desigualdade de Markov $\left\|p_{n}^{\prime}\right\|_{\infty} \leq n^{2}\left\|p_{n}\right\|_{\infty}$ para $p_{n} \in \Pi_{n}$, obtemos

$$
\begin{aligned}
& \left|L f(x)-Q_{n}(x)\right| \leq \int_{-1}^{1}\left|\left(\frac{k(x, t)-k(t, t)}{t-x}-\frac{P_{n}(x, t)-P_{n}(t, t)}{t-x}\right) f(t)\right| d t \leq \\
& \leq c\|f\|_{\infty, \alpha^{-}, \beta^{-}}\left\{b _ { n } \left(\int_{-1}^{x-(1+x) /\left(2 n^{2 m}\right)}+\int_{x+(1-x) /\left(2 n^{2 m}\right)}^{1} \frac{\omega^{-\alpha^{-},-\beta^{-}}(t)}{|t-x|} d t+\right.\right. \\
& \left.+\int_{x-(1+x) /\left(2 n^{2 m}\right)}^{x+(1-x) /\left(2 n^{2 m}\right)} \omega^{-\alpha^{-},-\beta^{-}}(t)|t-x|^{\mu-1} d t+c n^{2} \int_{x-(1+x) /\left(2 n^{2 m}\right)}^{x+(1-x) /\left(2 n^{2 m}\right)} \omega^{-\alpha^{-},-\beta^{-}}(t) d t\right\} .
\end{aligned}
$$

De [3], tem-se

$$
\left(\int_{-1}^{x-(1+x) /\left(2 n^{2}\right)}+\int_{x+(1-x) /\left(2 n^{2}\right)}^{1}\right) \frac{\omega^{-\alpha^{+},-\beta^{+}}(t)}{|t-x|} d t \leq c \omega^{-\alpha^{+},-\beta^{+}}(x) \log n,
$$

para $n \geq 2$. Desta desigualdade e do fato que $\omega^{-\alpha^{-},-\beta^{-}}(t) \leq c \omega^{-\alpha^{-},-\beta^{-}}(x)$ para $t \in\left[x-(1+x) /\left(2 n^{2}\right), x+(1-x) /\left(2 n^{2}\right)\right]$, obtemos

$$
\begin{aligned}
& \left|L f(x)-Q_{n}(x)\right| \leq c\|f\|_{\infty, \alpha^{-}, \beta^{-}} \omega^{-\alpha^{-},-\beta^{-}}(x)\left\{b_{n} \log n+c n^{2} \int_{x-(1+x) /\left(2 n^{2 m}\right)}^{x+(1-x) /\left(2 n^{2 m}\right)}|t-x|^{\mu-1} d t\right\} \leq \\
& \leq c\|f\|_{\infty, \alpha^{-}, \beta^{-}} \omega^{-\alpha^{-},-\beta^{-}}(x) b_{n} \log n .
\end{aligned}
$$

Logo,

$$
E_{n}^{\alpha^{-}, \beta^{-}}(f) \leq c\|f\|_{\infty, \alpha^{-}, \beta^{-}} b_{n} \log n \quad \text { e } \quad L \in \mathcal{L}\left(C_{\alpha^{-}, \beta^{-}}, C_{\alpha^{-}, \beta^{-}}^{\mathscr{B} \log n}\right)
$$

Proposição 3.2.8. Se $g(x) \in C_{\alpha^{+}, \beta^{+}}$, então $R g \in C_{\alpha^{-}, \beta^{-}}$.

Demonstração: Como $-\alpha^{-},-\beta^{-}>-1$, obtemos

$$
|(R g)(x)|=\left|\int_{-1}^{x} g(t) \omega^{\alpha, \beta}(t) d t\right| \leq c\|g\|_{\infty, \alpha^{+}, \beta^{+}} \int_{-1}^{x} \omega^{-\alpha^{-},-\beta^{-}}(t) d t \leq c\|g\|_{\infty, \alpha^{+}, \beta^{+}} .
$$

Então $R g \in C_{0,0}$. Pela Observação 3.1.6, obtemos $R g \in C_{\alpha^{-}, \beta^{-}}$.

Proposição 3.2.9. Sejam $a_{2}(x) \in C_{0,0}^{\mathscr{B}}$ e $R g(x) \in C_{\alpha^{-}, \beta^{-}}^{\mathscr{C}} \operatorname{com} \mathscr{B}=\left\{b_{n}\right\}, b_{n}=O\left(n^{-\gamma_{1}}\right)$ $e \mathscr{C}=\left\{c_{n}\right\}, c_{n}=O\left(n^{-\gamma_{2}}\right)$. Então,

$$
D \in \mathcal{L}\left(C_{\alpha^{-}, \beta^{-}}^{\mathscr{C}}, C_{\alpha^{-}, \beta^{-}}^{\mathscr{D}}\right)
$$

para $\mathscr{D}=\left\{d_{n}\right\}=O\left(n^{-\widetilde{\gamma}}\right)$ e $\widetilde{\gamma}=\min \left\{\gamma_{1}, \gamma_{2}\right\}$ 
$\underline{\text { Demonstração: }}$ Como $a_{2}, R g \in C_{0,0}$, obtemos $|(D R g)(x)| \leq c\|g\|_{\infty, \alpha^{+}, \beta^{+}}$. Então $D \in \mathcal{L}\left(C_{\alpha^{-}, \beta^{-}}, C_{0,0}\right)$, o que implica em $D \in \mathcal{L}\left(C_{\alpha^{-}, \beta^{-}}, C_{\alpha^{-}, \beta^{-}}\right)$.

Sejam $p_{n}^{*}(x), p_{n}^{* *}(x)$ tais que $E_{n}\left(a_{2}\right)=\left\|a_{2}-p_{n}^{*}\right\|_{\infty}$ e $E_{n}^{\alpha^{-}, \beta^{-}}(R g)=\left\|R g-p_{n}^{* *}\right\|_{\infty, \alpha^{-}, \beta^{-}}$. Então,

$$
\begin{aligned}
& E_{2 n}^{\alpha^{-}, \beta^{-}}(D g) \leq\left\|a_{2}(x) R g(x)-p_{n}^{*}(x) p_{n}^{* *}(x)\right\|_{\infty, \alpha^{-}, \beta^{-}} \leq \\
& \leq\left\|\left(a_{2}-p_{n}^{*}\right) R g\right\|_{\infty, \alpha^{-}, \beta^{-}}+\left\|\left(R g-p_{n}^{* *}\right) p_{n}^{*}\right\|_{\infty, \alpha^{-}, \beta^{-}} \leq c\left\{E_{n}\left(a_{2}\right)+E_{n}^{\alpha^{-}, \beta^{-}}(R g)\right\} \leq \\
& \leq c\left\{\left\|a_{2}\right\|_{0,0, \mathscr{B}} b_{n}+\|R g\|_{\alpha^{-}, \beta^{-}, \mathscr{C}} c_{n}\right\} \leq c d_{n} \leq c d_{2 n} .
\end{aligned}
$$

Observação 3.2.10. Nas condições da Proposição 3.2.6, para $\rho \leq \alpha^{-}$e $\tau \leq \beta^{-}$, obtemos $C_{\rho, \tau}^{\mathscr{B}} \subset C_{\alpha^{-}, \beta^{-}}^{\mathscr{B}} \mathrm{e}$

$$
L R \in \mathcal{L}\left(C_{\alpha^{+}, \beta^{+}}, C_{\alpha^{-}, \beta^{-}}^{\mathscr{B}}\right) \Rightarrow \widetilde{H} L R \in \mathcal{L}\left(C_{\alpha^{+}, \beta^{+}}, C_{\alpha^{-}, \beta^{-}}^{\mathscr{B} \log n}\right)
$$

Nas condições da Proposição 3.2.7, obtemos

$$
L R \in \mathcal{L}\left(C_{\alpha^{+}, \beta^{+}}, C_{\alpha^{-}, \beta^{-}}^{\mathscr{B} \log n}\right) \Rightarrow \widetilde{H} L R \in \mathcal{L}\left(C_{\alpha^{+}, \beta^{+}}, C_{\alpha^{-}, \beta^{-}}^{\mathscr{B} \log n}\right)
$$

e pela Proposição 3.2.9, obtemos

$$
D R \in \mathcal{L}\left(C_{\alpha^{+}, \beta^{+}}, C_{\alpha^{-}, \beta^{-}}^{\mathscr{D}}\right) \Rightarrow \widetilde{H} D R \in \mathcal{L}\left(C_{\alpha^{+}, \beta^{+}}, C_{\alpha^{-}, \beta^{-}}^{\mathscr{D} \log n}\right)
$$

\subsection{Convergência do método de colocação polinomial}

Nesta seção, encontram-se os principais resultados que utilizamos na prova da convergência do MCP, segundo a norma uniforme ponderada $\|\cdot\|_{\infty, \alpha^{+}, \beta^{+}}$. Primeiramente, apresentamos algumas considerações iniciais.

Seja o operador de projeção

$$
\begin{aligned}
\mathbb{P}_{n-1}:=C[-1,1] & \rightarrow C[-1,1] \\
f & \rightarrow\left(\mathbb{P}_{n-1} f\right)(x)=\sum_{i=1}^{n} f\left(x_{i}\right) l_{i}(x),
\end{aligned}
$$

onde $l_{i}$, para $1 \leq i \leq n$, denotam os polinômios fundamentais da interpolação de Lagrange correspondentes às abscissas $x_{i}$, que são os nós de interpolação. 
Lema 3.3.1. ([6], Lema 3.4.2) De acordo com as considerações anteriores, para $r_{n}(x)$ definida em (2.16), obtemos

$$
r_{n}(x)=0 \Leftrightarrow \mathbb{P}_{n-1} r_{n}(x)=0 .
$$

Aplicando este lema em $r_{n}$, definida em (2.16), temos

$$
\mathbb{P}_{n-1} H g_{n}+\mathbb{P}_{n-1} D R g_{n}+\mathbb{P}_{n-1} L R g_{n}=\mathbb{P}_{n-1} \bar{f}
$$

Pelo Lema 2.2.2, $H g_{n}$ é polinômio de grau $n-1$ e a equação anterior pode ser expressa por:

$$
H g_{n}+\mathbb{P}_{n-1} D R g_{n}+\mathbb{P}_{n-1} L R g_{n}=\mathbb{P}_{n-1} \bar{f}
$$

Apresentamos a seguir, alguns conceitos necessários ao enunciado do lema seguinte.

Definição 3.3.2. Um sistema de funções $\left\{\phi_{1}, \ldots, \phi_{n}\right\}$ é dito satizfazer a condição de Haar se $\phi_{i}, 1 \leq i \leq n$, é contínua, e se todo conjunto de $n$ vetores da forma $\left(\phi_{1}(x), \cdots, \phi_{n}(x)\right)$ é linearmente independente.

Definição 3.3.3. Seja a função $\psi_{j}$ tal que

$$
\sum_{j=0}^{n-1} \phi_{j}\left(x_{i}\right) \psi_{j}(t)=l\left(x_{i}, t\right), \quad 1 \leq i \leq n,
$$

onde $\left\{\phi_{j}\right\}$ é uma base para o conjunto de polinômios de grau $n-1$ satisfazendo a condição de Haar. Definimos o polinômio interpolador de $l(x, t)$ na variável $x$ como:

$$
l^{(n-1)}(x, t)=\sum_{j=0}^{n-1} \phi_{j}(x) \psi_{j}(t) .
$$

Lema 3.3.4. ([6], Lema 3.4.3) Sejam $l(x, t)$ e $l^{(n-1)}(x, t)$ conforme definido em (3.2). Seja também o operador

$$
L^{(n-1)} f(x)=\int_{-1}^{1} l^{(n-1)}(x, t) f(t) d t .
$$

Então,

$$
\mathbb{P}_{n-1} L=L^{(n-1)}
$$

Definição 3.3.5. A constante de Lebesgue ponderada é definida por:

$$
\left\|\mathbb{P}_{n-1}\right\|_{\infty, \rho, \tau}:=\sup \left\{\left\|\mathbb{P}_{n-1} f\right\|_{\infty, \rho, \tau}: f \in C_{\rho, \tau},\|f\|_{\infty, \rho, \tau}=1\right\}
$$


O lema a seguir, é apresentado em [12], Lema 5.2. Devido à necessidade de adaptação do seu enunciado para a demonstração do teorema de convergência do MCP, apresentamos sua demonstração com as devidas modificações.

Lema 3.3.6. Se $f \in C_{\rho, \tau}^{\mathscr{B}} \operatorname{com} \rho \leq \alpha^{-} e \tau \leq \beta^{-}$, então

$$
\left\|\widetilde{H}\left(f-\mathbb{P}_{n-1} f\right)\right\|_{\infty, \alpha^{+}, \beta^{+}} \leq c \log n\left\|\mathbb{P}_{n-1}\right\|_{\infty, \rho, \tau} E_{n-1}^{\alpha^{-}, \beta^{-}}(f), \quad n \geq 2 .
$$

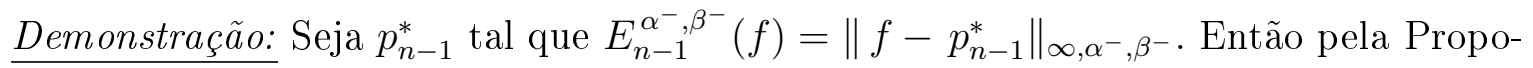
sição 3.2 .2 , obtemos

$$
\begin{aligned}
& \left\|\widetilde{H}\left(f-\mathbb{P}_{n-1} f\right)\right\|_{\infty, \alpha^{+}, \beta^{+}} \leq c\left[\left\|f-\mathbb{P}_{n-1} f\right\|_{\infty, \alpha^{-}, \beta^{-}} \log n+\frac{\left\|f-\mathbb{P}_{n-1} f\right\|_{\alpha^{-}, \beta^{-,}, \mathscr{B}}}{n^{\mu}}\right] \leq \\
& \leq c\left[\left(\left\|f-p_{n-1}^{*}\right\|_{\infty, \alpha^{-}, \beta^{-}}+\left\|\mathbb{P}_{n-1}\left(f-p_{n-1}^{*}\right)\right\|_{\infty, \alpha^{-}, \beta^{-}}\right) \log n+\sup _{m=1,2, \ldots} \frac{E_{m}^{\alpha^{-}, \beta^{-}}\left(f-\mathbb{P}_{n-1} f\right)}{n^{\mu}}\right] \leq \\
& \leq c\left[\left(E_{n-1}^{\alpha^{-}, \beta^{-}}(f)+\left\|\mathbb{P}_{n-1}\right\|_{\infty, \rho, \tau} E_{n-1}^{\alpha^{-}, \beta^{-}}(f)\right) \log n+\sup _{m=1,2, \ldots} \frac{E_{m}^{\alpha^{-}, \beta^{-}}\left(f-\mathbb{P}_{n-1} f\right)}{n^{\mu}}\right] \leq \\
& \leq c\left[\log n\left\|\mathbb{P}_{n-1}\right\|_{\infty, \rho, \tau} E_{n-1}^{\alpha^{-}, \beta^{-}}(f)+\sup _{m=1,2, \ldots} \frac{E_{m}^{\alpha^{-}, \beta^{-}}\left(f-\mathbb{P}_{n-1} f\right)}{n^{\mu}}\right] .
\end{aligned}
$$

Vejamos que

$E_{m}^{\alpha^{-}, \beta^{-}}\left(f-\mathbb{P}_{n-1} f\right)\left\{\begin{array}{lr}=E_{m}^{\alpha^{-}, \beta^{-}}(f) & \text { se } \\ \leq\left\|f-\mathbb{P}_{n-1}(f)\right\|_{\infty, \alpha^{-}, \beta^{-}} \leq c\left\|\mathbb{P}_{n-1}\right\|_{\infty, \rho, \tau} E_{n-1}^{\alpha^{-}, \beta^{-}}(f) & \text { se } \quad m<n-1 .\end{array}\right.$

Logo,

$$
\left\|\widetilde{H}\left(f-\mathbb{P}_{n-1} f\right)\right\|_{\infty, \alpha^{+}, \beta^{+}} \leq c \log n\left\|\mathbb{P}_{n-1}\right\|_{\infty, \rho, \tau} E_{n-1}^{\alpha^{-}, \beta^{-}}(f) .
$$

O próximo teorema, será utilizado na demonstração dos Lemas 3.3.8 e 3.3.11, adaptações dos Lemas 6.7 e 6.11 de [12].

Teorema 3.3.7. ([10, p. 328]) Se os nós de uma fórmula de quadratura interpolatória são os zeros do polinômio ortogonal de grau $n$ associado ao peso do termo $\lambda_{i}(t)$, então essa fórmula possui grau de precisão $2 n-1$.

Lema 3.3.8. Sejam $\nu$ e $\varsigma$ constantes não negativas tais que $\nu+\alpha^{-}<1$ e $\varsigma+\beta^{-}<1$. Se $l(x, t) \in C_{\rho, \tau, x}^{\mathscr{B}} \cap C_{\nu, \varsigma, t}$, ent $\tilde{a} o$

$$
\left\|\left(\widetilde{L^{n}}-L\right) R g_{n}\right\|_{\infty, \rho, \tau} \leq c E_{n}^{\nu, \varsigma}\left(l_{x}^{\rho, \tau}\right)
$$


Demonstração: Sabemos que

$$
\left(R g_{n}\right)(x)=\sum_{j=0}^{n} \int_{-1}^{x} c_{j} P_{j}^{\alpha, \beta}(t) \omega^{\alpha, \beta}(t) d t=-\sum_{j=1}^{n} \frac{c_{j}}{2 j} P_{j-1}^{\alpha+1, \beta+1}(x) \omega^{\alpha+1, \beta+1}(x)
$$

e portanto, $R g_{n}(t) \omega^{-\alpha-1,-\beta-1}(t)$ é um polinômio de grau $n-1$. Tomando $t_{i}, 1 \leq i \leq n$ como os zeros de $P_{n}^{\alpha+1, \beta+1}(t)$, obtemos

$$
\widetilde{L}^{n} R g_{n}=-\sum_{j=1}^{n} \frac{c_{j}}{2 j} \sum_{i=1}^{n} l\left(x, t_{i}\right) P_{j-1}^{\alpha+1, \beta+1}\left(t_{i}\right) \lambda_{i}^{\alpha+1, \beta+1},
$$

para $\lambda_{i}^{\alpha+1, \beta+1}(t)$ conforme definido em (2.18). Então, obtemos

$$
\begin{aligned}
\left|\left(\widetilde{L}^{n}-L\right) R g_{n} \omega^{\rho, \tau}(x)\right|=\mid \int_{-1}^{1}[ & \sum_{i=1}^{n} l\left(x, t_{i}\right) \omega^{\rho, \tau}(x) R g_{n}\left(t_{i}\right) \omega^{-\alpha-1,-\beta-1}\left(t_{i}\right) \lambda_{i}^{\alpha+1, \beta+1}- \\
& \left.-l(x, t) \omega^{\rho, \tau}(x) R g_{n}(t)\right] d t \mid .
\end{aligned}
$$

Seja $p_{2 n-1}^{*}$ tal que $E_{2 n-1}^{\nu, \varsigma}\left(l_{x}^{\rho, \tau} R g_{n} \omega^{-\alpha-1,-\beta-1}\right)=\left\|l_{x}^{\rho, \tau} R g_{n} \omega^{-\alpha-1,-\beta-1}-p_{2 n-1}^{*}\right\|_{\infty, \nu, \varsigma}$. Pelo Teorema 3.3.7, temos

$$
\begin{aligned}
& \left|\left(\widetilde{L}^{n}-L\right) R g_{n}(x) \omega^{\rho, \tau}(x)\right|= \\
& =\mid \int_{-1}^{1}\left\{\sum_{i=1}^{n}\left[l\left(x, t_{i}\right) \omega^{\rho, \tau}(x) R g_{n}\left(t_{i}\right) \omega^{-\alpha-1,-\beta-1}\left(t_{i}\right)-p_{2 n-1}^{*}\left(t_{i}\right)\right] \lambda_{i}^{\alpha+1, \beta+1}-\right. \\
& -\left[l(x, t) \omega^{\rho, \tau}(x) R g_{n}(t) \omega^{-\alpha-1,-\beta-1}(t)-p_{2 n-1}^{*}(t)\right] \omega^{\alpha+1, \beta+1}(t)- \\
& \left.-\left[p_{2 n-1}^{*}(t) \omega^{\alpha+1, \beta+1}(t)-p_{2 n-1}^{*}\left(t_{i}\right) \lambda_{i}^{\alpha+1, \beta+1}\right]\right\} \omega^{\nu, \varsigma}(t) \omega^{-\nu,-\varsigma}(t) d t \mid \leq \\
& \leq E_{2 n-1}^{\nu, \varsigma}\left(l_{x}^{\rho, \tau} R g_{n} \omega^{-\alpha-1,-\beta-1}\right) \int_{-1}^{1}\left\{\left|\lambda_{i}^{\alpha+1, \beta+1}\right|+\omega^{\alpha+1, \beta+1}(t)\right\} \omega^{-\nu,-\varsigma}(t) d t .
\end{aligned}
$$

Como por hipótese $\nu+\alpha^{-}<1$ e $\varsigma+\beta^{-}<1$, então $-\nu,-\varsigma>-1$, e isto resulta em $\int_{-1}^{1} \omega^{-\nu,-\varsigma}(t) d t$ ser limitada. Portanto,

$$
\left|\left(\widetilde{L}^{n}-L\right) R g_{n}(x) \omega^{\rho, \tau}(x)\right| \leq c E_{2 n-1}^{\nu, \varsigma}\left(l_{x}^{\rho, \tau} R g_{n} \omega^{-\alpha-1,-\beta-1}\right) .
$$

Seja $p_{n}^{*}$ tal que $E_{n}^{\nu, \varsigma}\left(l_{x}^{\rho, \tau}\right)=\left\|l_{x}^{\rho, \tau}-p_{n}^{*}\right\|_{\infty, \nu, \varsigma}$. Então, obtemos

$$
E_{2 n-1}^{\nu, \varsigma}\left(l_{x}^{\rho, \tau} R g_{n} \omega^{-\alpha-1,-\beta-1}\right) \leq\left\|\left(l_{x}^{\rho, \tau}-p_{n}^{*}\right) R g_{n} \omega^{-\alpha-1,-\beta-1}\right\|_{\infty, \nu, \varsigma} \leq c E_{n}^{\nu, \varsigma}\left(l_{x}^{\rho, \tau}\right) .
$$

Para a demonstração do Lema 3.3.11 utilizamos os dois teoremas seguintes.

Teorema 3.3.9. ([9], Teorema 8.4.8) Sejam $P_{n} \in \Pi_{n}$ e $w \in J_{\infty}^{*}$, classe de pesos que inclui os pesos de Jacobi $\omega^{\rho, \tau}(x)$, para $\rho, \tau \geq 0$. Então, para $0<c<n^{2}$,

$$
\left\|P_{n} w\right\|_{L^{\infty}[-1,1]} \leq M(c)\left\|P_{n} w\right\|_{L^{\infty}\left[-1+c n^{-2}, 1-c n^{-2}\right]},
$$

onde $M(c)$ é uma constante positiva independente de $n$ e $P_{n}$. 
Teorema 3.3.10. ([9], Teoremas 7.3.1) Sejam $r$ um inteiro positivo e $p_{n}$ tal que $E_{n}(f)=\inf _{p_{n} \in \Pi_{n}}\left\|f-p_{n}\right\|_{L^{p}[-1,1]}$. Então vale a desigualdade

$$
\left\|\phi p_{n}^{(r)}\right\|_{L^{p}[-1,1]} \leq M(r) \sum_{0 \leq k \leq n}(k+1)^{r-1} E_{k}(f)
$$

onde $\phi(x)=\sqrt{1-x^{2}}$.

Lema 3.3.11. Seja $l(x, t)=[k(x, t)-k(t, t)] /(t-x) \operatorname{com} k(x, t) \in C_{0,0, x}^{\mathscr{B}} \cap C_{0,0, t}^{\mathscr{B}} e$ $\mathscr{B}=\left\{b_{n}\right\}, b_{n}=O\left(n^{-\gamma}\right)$. Ent $\tilde{a} o$,

$$
\left\|\left(\widetilde{L}^{n}-L\right) R g_{n+1}\right\|_{\infty, \alpha^{-}, \beta^{-}} \leq c\left\|R g_{n+1} \omega^{-\alpha-1,-\beta-1}\right\|_{\infty} \begin{cases}1 / n & \text { se } \gamma>1 \\ \log n / n^{\gamma} & \text { se } \gamma \leq 1\end{cases}
$$

$\underline{\text { Demonstração: }}$ Definimos a quadratura de $L R g_{n}=\int_{-1}^{1}[k(x, t)-k(t, t)] /(t-x) R g_{n}(t) d t$ como sendo

$$
\widetilde{L}^{n}\left(R g_{n}\right):=\sum_{i \neq d=1}^{n} \frac{k\left(x, t_{i}\right)-k\left(t_{i}, t_{i}\right)}{t_{i}-x} R g_{n}\left(t_{i}\right) \omega^{-\alpha-1,-\beta-1}\left(t_{i}\right) \lambda_{i}^{\alpha+1, \beta+1},
$$

onde $\lambda_{i}^{\alpha+1, \beta+1}$ e $t_{i}$ são conforme definidos em (2.18), e $d=\left\{j:\left|x-t_{j}\right|=\min _{1 \leq i \leq n}\left|x-t_{i}\right|\right\}$.

Suponha que para cada $x \in[-1,1]$ fixado, $p_{n}^{*}$ seja tal que

$$
E_{n}^{0,0}\left[k_{x}^{0,0}(t)-k(t, t)\right]=\left\|\left[k_{x}^{0,0}-k(t, t)\right]-p_{n}^{*}\right\|_{\infty}
$$

Levando em conta o fato que $\left[k_{x}^{0,0}(t)-k(t, t)\right](x)=0$, obtemos

$$
\left\|k_{x}^{0,0}(t)-k(t, t)-p_{n}^{*}(t)-p_{n}^{*}(x)\right\|_{\infty} \leq c E_{n}^{0,0}\left[k_{x}^{0,0}(t)-k(t, t)\right]
$$

Então, para $k_{x}^{0,0} \in \mathcal{H}^{\mu}([-1,1])$ e $m \leq(\gamma+2) /(2 \mu)$, obtemos

$$
\begin{aligned}
& \left|\left(\widetilde{L}^{n}-L\right) R g_{n+1}(x)\right|=\mid \int_{-1}^{1} \sum_{i \neq d=1}^{n}\left\{\left[\frac{k_{x}^{0,0}(t)-k(t, t)}{t-x}-\frac{p_{n}^{*}(t)-p_{n}^{*}(x)}{t-x}\right] R g_{n+1}(t)-\right. \\
& \left.-\left[\frac{k_{x}^{0,0}\left(t_{i}\right)-k\left(t_{i}, t_{i}\right)}{t_{i}-x}-\frac{p_{n}^{*}\left(t_{i}\right)-p_{n}^{*}(x)}{t_{i}-x}\right] \omega^{-\alpha-1,-\beta-1}\left(t_{i}\right) R g_{n+1}\left(t_{i}\right) \lambda_{i}^{\alpha+1, \beta+1}\right\}- \\
& -\left(\sum_{i \neq d=1}^{n}-\sum_{i=1}^{n}\right) \frac{p_{n}^{*}\left(t_{i}\right)-p_{n}^{*}(x)}{t_{i}-x} R g_{n+1}\left(t_{i}\right) \omega^{-\alpha-1,-\beta-1}\left(t_{i}\right) \lambda_{i}^{\alpha+1, \beta+1} d t \mid \leq \\
& \leq c\left\|R g_{n+1} \omega^{-\alpha-1,-\beta-1}\right\|_{\infty}\left\{\int_{-1}^{1}\left|\frac{k_{x}^{0,0}(t)-k(t, t)}{t-x}-\frac{p_{n}^{*}(t)-p_{n}^{*}(x)}{t-x}\right| \omega^{\alpha+1, \beta+1}(t) d t+\right. \\
& \left.+E_{n}^{0,0}\left[k_{x}^{0,0}(t)-k(t, t)\right] \sum_{i \neq d=1}^{n}\left|\frac{\lambda_{i}^{\alpha+1, \beta+1}}{x-t_{i}}\right|+\left|\frac{p_{n}^{*}\left(t_{d}\right)-p_{n}^{*}(x)}{t_{d}-x}\right|\left|\lambda_{d}^{\alpha+1, \beta+1}\right|\right\} .
\end{aligned}
$$


Para tratar a última integral, vamos reescrevê-la como:

$$
\begin{aligned}
& \int_{x-(1+x) / 2 n^{2 m}}^{x+(1-x) / 2 n^{2 m}}\left|\frac{k_{x}^{0,0}(t)-k(t, t)}{t-x}-\frac{p_{n}^{*}(t)-p_{n}^{*}(x)}{t-x}\right| \omega^{\alpha+1, \beta+1}(t) d t+ \\
& +\left(\int_{-1}^{x-(1+x) / 2 n^{2 m}}+\int_{x+(1-x) / 2 n^{2 m}}^{1}\right)\left|\frac{k_{x}^{0,0}(t)-k(t, t)}{t-x}-\frac{p_{n}^{*}(t)-p_{n}^{*}(x)}{t-x}\right| \omega^{\alpha+1, \beta+1}(t) d t .
\end{aligned}
$$

Aplicando a desigualdade de Markov $\left\|p_{n}^{\prime}\right\|_{\infty} \leq n^{2}\left\|p_{n}\right\|_{\infty}$, temos

$$
\begin{aligned}
& \left|\left(\widetilde{L}^{n}-L\right) R g_{n+1}(x)\right| \leq c\left\|R g_{n+1} \omega^{-\alpha-1,-\beta-1}\right\|_{\infty}\left\{n^{2} \int_{x-(1+x) / 2 n^{2 m}}^{x+(1-x) / 2 n^{2 m}}|x-t|^{\mu-1} \omega^{\alpha+1, \beta+1}(t) d t+\right. \\
& +E_{n}^{0,0}\left[k_{x}^{0,0}(t)-k(t, t)\right]\left(\int_{-1}^{x-(1+x) / 2 n^{2 m}}+\int_{x+(1-x) / 2 n^{2 m}}^{1} \frac{\omega^{\alpha+1, \beta+1}(t)}{|x-t|}+\right. \\
& \left.+E_{n}^{0,0}\left[k_{x}^{0,0}(t)-k(t, t)\right] \sum_{i \neq d=1}^{n}\left|\frac{\lambda_{i}^{\alpha+1, \beta+1}}{x-t_{i}}\right|+\left|\frac{p_{n}^{*}\left(t_{d}\right)-p_{n}^{*}(x)}{t_{d}-x}\right|\left|\lambda_{d}^{\alpha+1, \beta+1}\right|\right\} .
\end{aligned}
$$

Usando o fato de

$$
\omega^{-\alpha^{-},-\beta^{-}}(t) \leq c \omega^{-\alpha^{-},-\beta^{-}}(x) \text { para } t \in\left[x-(1+x) /\left(2 n^{2 m}\right), x+(1-x) /\left(2 n^{2 m}\right)\right],
$$

e a desigualdade (3.1) (substituindo $n$ por $n^{m}$ e $\alpha^{+}, \beta^{+}$por $\alpha^{-}, \beta^{-}$), obtemos

$$
\begin{aligned}
& \left|\left(\widetilde{L}^{n}-L\right) R g_{n+1}(x)\right| \leq c\left\|R g_{n+1} \omega^{-\alpha-1,-\beta-1}\right\|_{\infty}\left\{\omega^{\alpha+1, \beta+1}(x) b_{n}+\right. \\
& +\left|\frac{p_{n}^{*}\left(t_{d}\right)-p_{n}^{*}(x)}{t_{d}-x}\right| \lambda_{d}^{\alpha+1, \beta+1} \mid+E_{n}^{0,0}\left[k_{x}^{0,0}(t)-k(t, t)\right]\left(\omega^{\alpha+1, \beta+1}(x) \log n+\right. \\
& \left.\left.+\sum_{i \neq d=1}^{n}\left|\frac{\lambda_{i}^{\alpha+1, \beta+1}}{x-t_{i}}\right|\right)\right\} .
\end{aligned}
$$

Pelo teorema do valor médio e o Teorema 3.3.9, obtemos:

$$
\left|\frac{p_{n}^{*}\left(t_{d}\right)-p_{n}^{*}(x)}{t_{d}-x}\right|=c\left|\left(p_{n}^{*}\right)^{\prime}(\xi)\right| \leq \sup _{|t| \leq 1-(2 n)^{-2}} c\left|\left(p_{n}^{*}\right)^{\prime}(t)\right| \leq c n\left\|\left(p_{n}^{*}\right)^{\prime}\right\|_{\infty, 1 / 2,1 / 2}
$$

para $\xi$ entre $x$ e $t_{d}$. Pelo Teorema 3.3.10, obtemos

$$
\left\|\left(p_{n}^{*}\right)^{\prime}\right\|_{\infty, 1 / 2,1 / 2} \leq c \sum_{0 \leq i \leq n} E_{i}\left[k_{x}^{0,0}(t)-k(t, t)\right] \leq c \sum_{i=1}^{n} b_{i},
$$

e do Teorema 9.22 de [20], segue

$$
\text { se } x \notin\left[t_{1}, t_{n}\right] \text { então } \sqrt{1-t_{d}^{2}} \leq \sqrt{2} \max \left\{\sqrt{1+t_{1}}, \sqrt{1-t_{n}}\right\} \leq c n^{-1} \text {, }
$$

portanto:

$$
\left|\frac{p_{n}^{*}\left(t_{d}\right)-p_{n}^{*}(x)}{t_{d}-x}\right| \leq c \frac{\sum_{1 \leq i \leq n} b_{i}}{\sqrt{1-t_{d}^{2}}}, \quad x \notin\left[t_{1}, t_{n}\right]
$$


Se $x \in\left[t_{1}, t_{n}\right]$, do Teorema 4.1 de [3], obtemos $\left|\xi-t_{d}\right| \leq c\left(\sqrt{1-\xi^{2}}+n^{-1}\right) n^{-1}$. Também de [20], Teorema 9.22, segue

$$
1 / n \leq c \sqrt{1-t_{n}} \leq c \sqrt{1-\xi}, 1 / n \leq c \sqrt{1+t_{1}} \leq c \sqrt{1+\xi}
$$

donde obtemos $\left|\xi-t_{d}\right| \leq c(1 \pm \xi)$ e consequentemente $1 \pm t_{d} \leq c(1 \pm \xi)$. Assim,

$$
\left|\frac{p_{n}^{*}\left(t_{d}\right)-p_{n}^{*}(x)}{t_{d}-x}\right| \leq c \frac{\sum_{1 \leq i \leq n} b_{i}}{\sqrt{1-t_{d}^{2}}}, \quad x \in[-1,1] .
$$

De [20], Teoremas 6.3.28 e 9.22, para $\alpha, \beta>-1$,

$$
\lambda_{i}^{\alpha, \beta} \leq c \frac{\omega^{\alpha+1 / 2, \beta+1 / 2}\left(t_{i}\right)}{n}, \quad 1 \leq i \leq n, n=1,2, \cdots
$$

Portanto, obtemos

$$
\left|\frac{p_{n}^{*}\left(t_{d}\right)-p_{n}^{*}(x)}{t_{d}-x}\right|\left|\lambda_{d}^{\alpha+1, \beta+1}\right| \leq c \frac{\omega^{\alpha+1, \beta+1}\left(t_{d}\right)}{n} \sum_{i=1}^{n} b_{i}
$$

Pelo Lema 4.1 de [15], tem-se para $-1 / 2 \leq \gamma, \delta \leq 1 / 2$,

$$
\sum_{i \neq d=1}^{n} \frac{\left(1-t_{i}\right)^{\gamma}\left(1+t_{i}\right)^{\delta}}{n\left|t-t_{i}\right|} \leq c \omega_{n}^{\gamma-1 / 2, \delta-1 / 2}(t) \log n,|t| \leq 1
$$

onde

$$
\omega_{n}^{\gamma-1 / 2, \delta-1 / 2}(t)=\left(\sqrt{1-t}-\frac{1}{n}\right)^{2 \gamma-1}\left(\sqrt{1+t}+\frac{1}{n}\right)^{2 \delta-1} .
$$

Então, como $\omega^{\gamma, \delta}(t) \leq c \omega_{n}^{\gamma, \delta}(t)$ para $\gamma, \delta \geq 0$, obtemos

$$
\begin{aligned}
\sum_{i \neq d=1}^{n}\left|\frac{\lambda_{i}^{\alpha+1, \beta+1}(t)}{x-t_{i}}\right| & \leq c \sum_{i \neq d=1}^{n} \frac{\omega^{\alpha+3 / 2, \beta+3 / 2}\left(t_{i}\right)}{n\left|x-t_{i}\right|} \leq c \sum_{i \neq d=1}^{n} \frac{\omega^{-\alpha^{-}+1 / 2,-\beta^{-}+1 / 2}\left(t_{i}\right)}{n\left|x-t_{i}\right|} \leq \\
& \leq c \log n \omega_{n}^{-\alpha^{-},-\beta^{-}}(x) \leq c \log n \omega^{-\alpha^{-},-\beta^{-}}(x) .
\end{aligned}
$$

Portanto,obtemos

$$
\begin{aligned}
& \left|\left(\widetilde{L}^{n}-L\right) R g_{n+1}(x)\right| \leq c\left\|R g_{n+1} \omega^{-\alpha-1,-\beta-1}\right\|_{\infty}\left\{b_{n} \omega^{, \alpha+1, \beta+1}(x)+\frac{\omega^{\alpha+1, \beta+1}\left(t_{d}\right)}{n} \sum_{i=1}^{n} b_{i}+\right. \\
& +E_{n}^{0,0}\left[k_{x}^{0,0}(t)-k(t, t)\right]\left(\omega^{\alpha+1, \beta+1}(x) \log n+\omega^{-\alpha^{-},-\beta^{-}}(x) \log n\right) \leq \\
& \leq c\left\|R g_{n+1} \omega^{-\alpha-1,-\beta-1}\right\|_{\infty}\left\{b_{n} \omega^{-\alpha^{-},-\beta^{-}}(x) \log n+\frac{\omega^{\alpha+1, \beta+1}\left(t_{d}\right)}{n} \sum_{i=1}^{n} b_{i}\right\} .
\end{aligned}
$$

Como $\omega^{\alpha+1, \beta+1}(x) \leq c \omega^{\alpha+1, \beta+1}\left(t_{d}\right)$, obtemos

$$
\left\|\left(\widetilde{L}^{n}-L\right) R g_{n+1}\right\|_{\infty, \alpha^{-}, \beta^{-}} \leq c\left\|R g_{n+1} \omega^{-\alpha-1,-\beta-1}\right\|_{\infty}\left(\frac{\log n}{n^{\gamma}}+\frac{\sum_{1 \leq i \leq n} b_{i}}{n}\right) .
$$


Em [1, p.68], pode ser encontrada a identidade $\lim _{n \rightarrow \infty}\left(\sum_{k=1}^{n} 1 / k-\log n\right)=\gamma$, onde $\gamma \sim 0.5772 \ldots$ é a constante de Euler-Mascheroni. Então, temos

$$
\sum_{i=1}^{n} b_{i} \leq \begin{cases}c & \text { se } \gamma>1 \\ c \log n & \text { se } \gamma=1 \\ c \int_{1}^{n} 1 / t^{\gamma} d t \leq c n^{1-\gamma} & \text { se } \gamma<1\end{cases}
$$

e

$$
\left\|\left(\widetilde{L}^{n}-L\right) R g_{n+1}\right\|_{\infty, \alpha^{-}, \beta^{-}} \leq c\left\|R g_{n+1} \omega^{-\alpha-1,-\beta-1}\right\|_{\infty} \begin{cases}1 / n & \text { se } \gamma>1 \\ \log n / n^{\gamma} & \text { se } \gamma \leq 1\end{cases}
$$

No que se segue, considere o seguinte espaço de funções.

Definição 3.3.12. Denotamos por $W_{\gamma, \delta}^{s}$, o espaço de Sobolev ponderado definido por

$$
W_{\gamma, \delta}^{s}:=\left\{f \in C_{\gamma, \delta}: f^{(s-1)} \in A . C \cdot l o c \text { e }\left\|f^{(s)} \phi^{s} \omega^{\gamma, \delta}\right\|_{\infty}<\infty\right\}
$$

onde $f^{(s-1)} \in A . C \cdot$ loc significa que $f$ é $s-1$ vezes diferenciável e $f^{(s-1)}$ é absolutamente contínua em todo intervalo fechado $[c, d] \subset(-1,1)$. Equipamos o espaço $W_{\gamma, \delta}^{s}$ com a norma

$$
\|f\|_{W_{\gamma, \delta}^{s}}=\left\|f \omega^{\gamma, \delta}\right\|_{\infty}+\left\|f^{(s)} \phi^{s} \omega^{\gamma, \delta}\right\|_{\infty}
$$

O próximo teorema, apresenta a taxa de convergência da melhor aproximação polinomial na norma ponderada.

Teorema 3.3.13. ([9], Estimativa do tipo Jackson) Seja $f \in W_{\gamma, \delta}^{s}$ com s e n inteiros positivos. Então,

$$
E_{n}^{\gamma, \delta}(f) \leq \frac{c}{n^{s}} E_{n-s}^{\gamma+s / 2, \delta+s / 2}\left(f^{(s)}\right) \leq \frac{c}{n^{s}}\left\|f^{(s)} \phi^{s} \omega^{\gamma, \delta}\right\|_{\infty} .
$$

Notação. Denotamos por $W_{\gamma, \delta}^{s, \mu}$, o subconjunto de $W_{\gamma, \delta}^{s}$ definido por:

$$
W_{\gamma, \delta}^{s, \mu}:=\left\{f \in W_{\gamma, \delta}^{s}: f^{(s)} \phi^{s} \omega^{\gamma, \delta} \in \mathcal{H}^{\mu}([-1,1])\right\}
$$

Definição 3.3.14. Definimos a função módulo de continuidade de $f$ por:

$$
\Omega_{\delta}(f)=\sup _{d(x, y) \leq \delta}|f(x)-f(y)|,
$$

onde $f$ é uma função a valores reais definida em $[-1,1]$. 
Lema 3.3.15. Seja $f \in W_{\gamma, \delta}^{s, \mu}$. Então,

$$
E_{n}^{\gamma, \delta}(f) \leq \frac{c}{n^{s+\mu}}\left\|f^{(s)} \phi^{s} \omega^{\gamma, \delta}\right\|_{\infty}
$$

$\underline{\text { Demonstração: }}$ Definimos $\Upsilon(x)=n \int_{x(1-1 / n)-1 / 2 n}^{x(1-1 / n)+1 / 2 n} f^{(s)}(t) \phi^{s}(t) \omega^{\gamma, \delta}(t) d t$. Então, $\left|\Upsilon(x)-f^{(s)}(x) \phi^{s}(x) \omega^{\gamma, \delta}(x)\right|=$ $=n\left|\int_{x(1-1 / n)-1 / 2 n}^{x(1-1 / n)+1 / 2 n} f^{(s)}(t) \phi^{s}(t) \omega^{\gamma, \delta}(t)-f^{(s)}(x) \phi^{s}(x) \omega^{\gamma, \delta}(x) d t\right| \leq c \Omega_{3 / 2 n}\left(f^{(s)} \phi^{s} \omega^{\gamma, \delta}\right)$.

Sejam $S^{\prime}(x)=f^{(s)}(x) \phi^{s}(x) \omega^{\gamma, \delta}(x)$ e $0<\theta<1 / 2 n$. Então, obtemos

$$
\begin{aligned}
& |\Upsilon(x)|=n\left|\int_{x(1-1 / n)-1 / 2 n}^{x(1-1 / n)+1 / 2 n} S^{\prime}(t) d t\right|=n|S(x-x / n+1 / 2 n)-S(x-x / n-1 / 2 n)|= \\
& =n|S(x-x / n+1 / 2 n)-S(x-x / n)+S(x-x / n)-S(x-x / n-1 / 2 n)|= \\
& =n\left|S^{\prime}(x-x / n+\theta)-S^{\prime}(x-x / n-\theta)\right| / 2 n \leq c \Omega_{1 / n}\left(S^{\prime}\right) .
\end{aligned}
$$

Destes resultados e do Teorema 3.3.13, obtemos:

$$
\begin{aligned}
E_{n}^{\gamma, \delta}(f) & \leq \frac{c}{n^{s}}\left\{\left\|f^{(s)} \phi^{s} \omega^{\gamma, \delta}-\Upsilon\right\|_{\infty}+\|\Upsilon\|_{\infty}\right\} \leq \\
& \leq \frac{c}{n^{s}}\left\{\Omega_{3 / 2 n}\left(f^{(s)} \phi^{s} \omega^{\gamma, \delta}\right)+\Omega_{1 / n}\left(f^{(s)} \phi^{s} \omega^{\gamma, \delta}\right)\right\} \leq \frac{c}{n^{s+\mu}}
\end{aligned}
$$

No entanto, o Teorema 3.3.13 não é válido para $s=0$. Neste caso, devemos considerar o seguinte:

$$
\begin{aligned}
\left|\Upsilon^{\prime}(x)\right| & =n\left|\left(f \omega^{\gamma, \delta}\right)(x-x / n+1 / 2 n)-\left(f \omega^{\gamma, \delta}\right)(x-x / n-1 / 2 n)\right| \leq \\
& \leq c n \Omega_{1 / n}\left(f \omega^{\gamma, \delta}\right),
\end{aligned}
$$

e $E_{n}^{\gamma, \delta}(\Upsilon)=\left\|\left(\Upsilon-p_{n}^{*}\right) \omega^{\gamma, \delta}\right\|_{\infty}$ para algum $p_{n}^{*} \in \Pi_{n}$. Portanto, obtemos:

$$
\begin{aligned}
E_{n}^{\gamma, \delta}(f) & \leq c\left\{\left\|f \omega^{\gamma, \delta}-\Upsilon\right\|_{\infty}+\left\|\Upsilon\left(1-\omega^{\gamma, \delta}\right)\right\|_{\infty}+\left\|\left(\Upsilon-p_{n}^{*}\right) \omega^{\gamma, \delta}\right\|_{\infty}\right\} \leq \\
& \leq c\left\{\Omega_{3 / 2 n}\left(f \omega^{\gamma, \delta}\right)+\Omega_{1 / n}\left(f \omega^{\gamma, \delta}\right)+\frac{1}{n}\left\|\Upsilon^{\prime}(x) \phi(x) \omega^{\gamma, \delta}(x)\right\|_{\infty}\right\} \leq \\
& \leq c\left\{\Omega_{3 / 2 n}\left(f \omega^{\gamma, \delta}\right)+\Omega_{1 / n}\left(f \omega^{\gamma, \delta}\right)\right\} \leq \frac{c}{n^{\mu}} .
\end{aligned}
$$

Lema 3.3.16. Se $g \in C_{\alpha^{+}, \beta^{+}}$, a função $R g \in \mathcal{H}^{q}([-1,1])$ para $q=\min \left\{1-\alpha^{-}, 1-\beta^{-}\right\}$.

Demonstração: Seja $h>0$. Então, temos

$$
\begin{aligned}
& |R g(x+h)-R g(x)|= \\
& =\left|\int_{-1}^{x+h} g(t) \omega^{\alpha, \beta}(t) d t-\int_{-1}^{x} g(t) \omega^{\alpha, \beta}(t) d t\right| \leq\|g\|_{\infty, \alpha^{+}, \beta^{+}} \int_{x}^{x+h} \omega^{-\alpha^{-},-\beta^{-}}(t) d t \leq \\
& \leq c 2^{1-\alpha^{-}-\beta^{-}}\left\{\frac{B_{\frac{1+x+h}{2}}}{2}\left(1-\beta^{-}, 1-\alpha^{-}\right)-B_{\frac{1+x}{2}}\left(1-\beta^{-}, 1-\alpha^{-}\right)\right\},
\end{aligned}
$$


onde $B_{x}(a, b)$ é a função beta incompleta. De [1], equações 6.6.8 e 15.3.3, obtemos

$$
\begin{aligned}
& B_{x}(a, b)=a^{-1} x_{2}^{a}{ }_{2} F_{1}[a, 1-b ; a+1 ; x] \mathrm{e} \\
& { }_{2} F_{1}[a, b ; c ; z]=(1-z)^{c-a-b}{ }_{2} F_{1}[c-a, c-b ; c ; z],
\end{aligned}
$$

onde ${ }_{2} F_{1}(a, b ; c ; z)$ é a função hipergeométrica de Gauss. A partir destas identidades, obtemos

$$
\begin{aligned}
& B_{\frac{1+x}{2}}\left(1-\beta^{-}, 1-\alpha^{-}\right)=\left(1-\beta^{-}\right)^{-1}\left(\frac{1+x}{2}\right)^{1-\beta^{-}}{ }_{2} F_{1}\left[1-\beta^{-}, \alpha^{-} ; 2-\beta^{-} ;(1+x) / 2\right]= \\
& =\left(1-\beta^{-}\right)^{-1}\left(\frac{1+x}{2}\right)^{1-\beta^{-}}\left(\frac{1-x}{2}\right)^{1-\alpha^{-}}{ }_{2} F_{1}\left[1,2-\beta^{-}-\alpha^{-} ; 2-\beta^{-} ;(1+x) / 2\right]= \\
& =2^{-2+\beta^{-}+\alpha^{-}}\left(1-\beta^{-}\right)^{-1} \omega^{1-\beta^{-}, 1-\alpha^{-}}(x)_{2} F_{1}\left[1,2-\beta^{-}-\alpha^{-} ; 2-\beta^{-} ;(1+x) / 2\right] .
\end{aligned}
$$

Por definição

$$
{ }_{2} F_{1}[a, b ; c ; z]=\sum_{n=0}^{\infty} \frac{(a)_{n}(b)_{n}}{(c)_{n}} \frac{z^{n}}{n !}
$$

onde $(a)_{n}=\Gamma(a+n) / \Gamma(a)$ é o símbolo de Pochhammer. Disto, para algum $0<\theta<1$ obtemos

$$
\begin{aligned}
& \left|{ }_{2} F_{1}\left[1,2-\beta^{-}-\alpha^{-} ; 2-\beta^{-} ;(1+x+h) / 2\right]-{ }_{2} F_{1}\left[1,2-\beta^{-}-\alpha^{-} ; 2-\beta^{-} ;(1+x) / 2\right]\right|= \\
& =\left|\sum_{n=0}^{\infty} \frac{(1)_{n}\left(2-\beta^{-}-\alpha^{-}\right)_{n}}{\left(2-\beta^{-}\right)_{n}} \frac{(1+x+h)^{n}-(1+x)^{n}}{2^{n} n !}\right|= \\
& =\left|\sum_{n=0}^{\infty} \frac{(1)_{n}\left(2-\beta^{-}-\alpha^{-}\right)_{n}}{\left(2-\beta^{-}\right)_{n}} \frac{h\left[(1+x+\theta h)^{n}\right]^{\prime}}{2^{n} n !}\right|= \\
& =\left|\sum_{n=1}^{\infty} \frac{(1)_{n}\left(2-\beta^{-}-\alpha^{-}\right)_{n}}{\left(2-\beta^{-}\right)_{n}} \frac{h}{2 \Gamma(n)}\left(\frac{1+x+\theta h}{2}\right)^{n-1}\right| \leq \\
& \leq \frac{h}{2} \sum_{n=1}^{\infty} \frac{\Gamma(n+1) \Gamma\left(n+2-\beta^{-}-\alpha^{-}\right) \Gamma\left(2-\beta^{-}\right)}{\Gamma\left(n+2-\beta^{-}\right) \Gamma\left(2-\beta^{-}-\alpha^{-}\right) \Gamma(n)}=\frac{h}{2} \frac{\left(1-\beta^{-}\right)\left(2-\alpha^{-}-\beta^{-}\right)}{\left(1-\alpha^{-}\right)\left(2-\alpha^{-}\right)} \leq c h .
\end{aligned}
$$

De [18, p. 12], se $\sigma_{1} \neq \sigma_{2}$ são números positivos e $0 \leq \mu \leq 1$, então $\left|\sigma_{1}^{\mu}-\sigma_{2}^{\mu}\right| \leq\left|\sigma_{1}-\sigma_{2}\right|^{\mu}$. Portanto, $\omega^{1-\beta^{-}, 1-\alpha^{-}} \in \mathcal{H}([-1,1])^{q}$ onde $q=\min \left\{1-\alpha^{-}, 1-\beta^{-}\right\}$, e a função

$$
B_{\frac{1+x}{2}}\left(1-\beta^{-}, 1-\alpha^{-}\right) \in \mathcal{H}^{q}([-1,1]) .
$$

Logo, $R g \in \mathcal{H}^{q}([-1,1])$.

No próximo lema, são verificadas as condições necessárias para que a função $R g$ pertença a $W_{\alpha^{-}, \beta^{-}}^{s}$ para o maior $s$ possível, e a qual $\mathcal{H}^{q^{\prime}}([-1,1]), R g^{(s)} \phi^{s} \omega^{\alpha^{-}, \beta^{-}}$pertence. 
Lema 3.3.17. Se $g \in C_{\alpha^{+}, \beta^{+}}$, ent $\tilde{a} o R g \in W_{\alpha^{-}, \beta^{-}}^{s, q^{\prime}}$ para

$$
\begin{aligned}
& q^{\prime}=\min \left\{1-\alpha^{-}, 1-\beta^{-}, 1 / 2, \alpha_{0}^{-}, \beta_{0}^{-}\right\} \text {e } \\
& s=\left\{\begin{array}{l}
2 \quad \text { se }\left(\bar{f}-a_{2}\right)^{\prime} \in C_{\alpha^{-}+1, \beta^{-+1}} \text { e } \partial l(x, t) / \partial x \text { satifaz as condições da proposição } \\
\quad(3.2 .6) \operatorname{com} \rho \leq 1+\alpha^{-}, \tau \leq 1+\beta^{-}, \\
1 \quad \text { caso contrário. }
\end{array}\right.
\end{aligned}
$$

Demonstração: Primeiramente, verifiquemos o maior valor de $s$ para o qual $R g^{(s)} \in W_{\alpha^{-}, \beta^{-}}^{s}$ É claro que $R g \in W_{\alpha^{-}, \beta^{-}}^{1}$ mas,

$$
\begin{aligned}
R g^{(2)}(x) & =g^{\prime}(x) \omega^{\alpha, \beta}(x)+g(x) \omega^{\alpha-1, \beta-1}(x)[\beta-\alpha-(\alpha+\beta) x] . \\
& \therefore \quad R g \in W_{\alpha^{-}, \beta^{-}}^{2, q} \text { se } g^{\prime} \text { existir e }\left\|g^{\prime} \omega^{\alpha^{+}, \beta^{+}} \phi^{2}\right\|_{\infty}<\infty .
\end{aligned}
$$

No entanto, mesmo que $R g \in W_{\alpha^{-}, \beta^{-}}^{2}$,

$$
\begin{aligned}
& R g^{(3)}(x)=g^{(2)}(x) \omega^{\alpha, \beta}(x)+2 g^{\prime}(x) \omega^{\alpha-1, \beta-1}(x)[\beta-\alpha-x]+ \\
& +g(x) \omega^{\alpha-2, \beta-2}(x)\left\{\beta(\beta-1)(x-1)^{2}+\alpha(1+x)[-1+2 \beta(x-1)-x]+\alpha^{2}(1+x)^{2}\right\} \\
& \therefore R g^{(3)} \notin W_{\alpha^{-}, \beta^{-}}^{3}, \text { em geral. }
\end{aligned}
$$

Então $s$ é no máximo igual a 2. Agora, vamos verificar as condições necessárias para que isto ocorra. Pela equação (2.6), obtemos

$$
\begin{aligned}
a_{1}\left(\omega^{\alpha, \beta}(x) g(x)\right)^{\prime} & =\bar{f}^{\prime}-a_{2}(x)^{\prime} R g(x)-a_{2}(x) \omega^{\alpha, \beta}(x) g(x)-\int_{-1}^{1} \frac{\partial}{\partial x} l(x, t) R g(t) d t- \\
& -\frac{b_{1}}{\pi} \frac{\partial}{\partial x}\left(f_{-1}^{1} \frac{\omega^{\alpha, \beta}(t) g(t)}{t-x} d t\right) .
\end{aligned}
$$

Se fazemos uma integração por partes na última integral da equação (3.4), e em seguida aplicamos o Lema 3.3.16, obtemos:

$$
\begin{aligned}
& \left|\frac{\partial}{\partial x}\left(f_{-1}^{1} \frac{\omega^{\alpha, \beta}(t) g(t)}{t-x} d t\right)\right|=\left|f_{-1}^{1} \frac{\left(\omega^{\alpha, \beta} g\right)(t)}{(t-x)^{2}} d t\right|=2\left|f_{-1}^{1} \frac{R g(t)}{(t-x)} d t\right| \leq \\
& \leq 2\left|f_{-1}^{1} \frac{R g(t)-R g(x)}{(t-x)} d t\right|+2\left|R g(x) f_{-1}^{1} \frac{1}{(t-x)} d t\right| \leq c\left[(1-x)^{q}+(1+x)^{q}\right]+ \\
& +2 R g(x) \log \left|\frac{1-x}{1+x}\right|, \text { onde } q=\min \left\{1-\alpha^{-}, 1-\beta^{-}\right\} .
\end{aligned}
$$

Portanto, obtemos

$$
\left\|\phi^{2}(x) \omega^{\alpha^{-}, \beta^{-}}(x) \frac{\partial}{\partial x}\left(f_{-1}^{1} \frac{\omega^{\alpha, \beta}(t) g(t)}{t-x} d t\right)\right\|_{\infty}<\infty e \frac{\partial}{\partial x}\left(f_{-1}^{1} \frac{\omega^{\alpha, \beta}(t) g(t)}{t-x} d t\right) \in A C(-1,1),
$$


pois pela teoria do Capítulo 1, a derivada da integral em questão satisfaz a condição $\mathcal{H}([-1,1])$.

Pela Proposição 3.2.6, se $\partial l(x, t) / \partial x \in C_{\rho, \tau, x} \cap C_{\nu, \zeta, t} \operatorname{com} \nu+\alpha^{-}, \varsigma+\beta^{-}<1$, obtemos $\partial(L R g) / \partial x \in C_{\rho, \tau}$. Neste caso, se $\rho \leq \alpha^{-}+1, \tau \leq \beta^{-}+1$, tem-se

$$
\left\|\phi^{2}(x) \omega^{\alpha^{-}, \beta^{-}}(x) \int_{-1}^{1} \frac{\partial}{\partial x} l(x, t) R g(t) d t\right\|_{\infty} \text { limitado. }
$$

Pela Proposição 3.2.7, se $l(x, t)=[k(x, t)-k(t, t)] /(t-x) \operatorname{com} k(x, t) \in C_{0,0, x} \cap C_{0,0, t}$, $L R g \in C_{\alpha^{-}, \beta^{-}}$. Se $\partial l(x, t) / \partial x=\partial^{2} k(x, t) / \partial x^{2}$ existe e satisfaz as condições da Proposição 3.2.6, podemos avaliar a limitação de $\left\{[\partial(L R g) / \partial x] \phi^{2} \omega^{\alpha^{-}, \beta^{-}}\right\}(x)$ conforme anteriormente.

Logo, $R g \in W_{\alpha^{-}, \beta^{-}}^{s}$ onde

$$
s=\left\{\begin{aligned}
& 2 \text { se }\left(\bar{f}-a_{2}\right)^{\prime} \in C_{\alpha^{-}+1, \beta^{-}+1} \text { e } \partial l(x, t) / \partial x \text { satifaz as condições da Proposição } \\
& \quad 3.2 .6 \operatorname{com} \rho \leq 1+\alpha^{-}, \tau \leq 1+\beta^{-} \\
& 1 \quad \text { caso contrário. }
\end{aligned}\right.
$$

Pelo Teorema 3.3.13 e o Lema 3.1.8, $R g^{(s)} \phi^{s} \omega^{\alpha^{-}, \beta^{-}} \in \mathcal{H}^{q}([-1,1])$ para algum $0<q \leq 1$. Uma propriedade das funções que satisfazem a condição de Hölder, (veja [18, p. 16]), afirma que: para uma função $u(s) \in \mathcal{H}^{\mu}$ em algum intervalo $s_{1} \leq s \leq s_{2}$, se $f(u)$ é definida para valores de u neste mesmo intervalo, tal que $f^{\prime}(u)$ é limitada, então $f(u) \in \mathcal{H}^{\mu}$. Se aplicamos esta propriedade em $f\left(R g^{(1)} \phi \omega^{\alpha^{-}, \beta^{-}}\right)=\int_{-1}^{x}\left[R g^{(1)} \phi \omega^{\alpha^{-}, \beta^{-}}\right](t) d t$, como

$$
\begin{aligned}
& \int_{-1}^{x}\left[R g^{(1)} \phi \omega^{\alpha^{-}, \beta^{-}}\right](t) d t=\left(R g \phi \omega^{\alpha^{-}, \beta^{-}}\right)(x)-\int_{-1}^{x} R g(t)\left[\omega^{\alpha^{-}, \beta^{-}}(t) \phi(t)\right]^{\prime} d t= \\
& =\left(R g \phi \omega^{\alpha^{-}, \beta^{-}}\right)(x)-\left(R g \omega^{\alpha^{-}, \beta^{-}}\right)(\xi)\left[\beta^{-}-\alpha^{-}-\left(\alpha^{-}+\beta^{-}+1\right) \xi\right] \int_{-1}^{x} \phi^{-1}(t) d t= \\
& =\left(R g \phi \omega^{\alpha^{-}, \beta^{-}}\right)(x)+c B_{\frac{1+x}{2}}(1 / 2,1 / 2),
\end{aligned}
$$

concluímos que $R g^{(1)} \phi \omega^{\alpha^{-}, \beta^{-}} \in \mathcal{H}^{q^{\prime}}([-1,1])$, onde $q^{\prime}=\min \left\{1-\alpha^{-}, 1-\beta^{-}, 1 / 2, \alpha_{0}^{-}, \beta_{0}^{-}\right\}$. Procedendo de forma análoga com o termo $R g^{(2)} \phi^{2} \omega^{\alpha^{-}, \beta^{-}}$, obtemos:

$$
\begin{aligned}
& \int_{-1}^{x}\left[R g^{(2)} \phi^{2} \omega^{\alpha^{-}, \beta^{-}}\right](t) d t=\left(R g^{(1)} \phi^{2} \omega^{\alpha^{-}, \beta^{-}}\right)(x)-\int_{-1}^{x} R g^{(1)}(t)\left[\phi^{2}(t) \omega^{\alpha^{-}, \beta^{-}}(t)\right]^{\prime} d t= \\
& =\left(R g^{(1)} \phi^{2} \omega^{\alpha^{-}, \beta^{-}}\right)(x)-\left[R g^{(1)} \phi \omega^{\alpha^{-}, \beta^{-}}\right](\xi)\left[-2 \xi+\beta^{-}(1-\xi)-\alpha^{-}(1+\xi)\right] \int_{-1}^{x} \phi^{-1}(t) d t,
\end{aligned}
$$

que nos leva a concluir que $R g^{(2)} \phi^{2} \omega^{\alpha^{-}, \beta^{-}} \in \mathcal{H}^{q^{\prime}}([-1,1])$.

Observe que devido às condições $-1<\alpha, \beta<0$ serem verdadeiras quando $\varkappa=1$, obtemos $0<\alpha^{-}, \beta^{-}<1$ e portanto, $\alpha_{0}^{-}=\alpha^{-}$e $\beta_{0}^{-}=\beta^{-}$. Então neste caso, 
$q^{\prime}=\min \left\{1-\alpha^{-}, 1-\beta^{-}, 1 / 2\right\}$. Além disso, este lema nos diz que mesmo a função $g$ sendo desconhecida, a partir das funções $a_{2}, \bar{f}$ e $l$ podemos indicar uma estimativa do tipo Jackson para o termo $R g$, e esta estimativa é no máximo $5 / 2$.

Para demonstrarmos a convergência do MCP é necessário mostrar que o operador linear $\left[I+\widetilde{H} \mathbb{P}_{n-1}(\Gamma R+L R)\right]^{-1}$ é limitado. Para isto, utilizamos o próximo lema provado em [2, p. 15].

Lema 3.3.18. Sejam $X$ e $Y$ espaços de Banach e $T, S: X \rightarrow Y$ operadores lineares limitados, tais que $S^{-1}$ existe e $\|T-S\|\left\|S^{-1}\right\|<1$. Então,

$$
\left\|T^{-1}\right\| \leq \frac{\left\|S^{-1}\right\|}{1-\left\|S^{-1}\right\|\|T-S\|} .
$$

Lema 3.3.19. Suponha que o operador $I+\widetilde{H}(D+L) R$ seja continuamente inversivel em $C_{\alpha^{-}, \beta^{-}}$, e as condições das Proposições 3.2.6 ou 3.2.7 e 3.2.9 sejam satisfeitas. Então, para $n$ suficientemente grande,

$$
\begin{aligned}
& \left\|\left[I+\widetilde{H} \mathbb{P}_{n-1}(D R+L R)\right]^{-1}\right\|_{\infty, \alpha^{+}, \beta^{+}} \leq \\
& \leq \frac{\left\|[I+\widetilde{H}(D+L) R]^{-1}\right\|_{\infty, \alpha^{+}, \beta^{+}}}{1-\left\|[I+\widetilde{H}(D+L) R]^{-1}\right\|_{\infty, \alpha^{+}, \beta^{+}}\left\|\widetilde{H}\left[\mathbb{P}_{n-1}(D+L)-(D+L)\right] R\right\|_{\infty, \alpha^{-}, \beta^{-}}} .
\end{aligned}
$$

Demonstração: Pelas Proposições 3.2.4, 3.2.6 ou 3.2.7 e 3.2.9, o operador $\widetilde{H}(D+L) R g$ e consequentemente o operador $\widetilde{H} \mathbb{P}_{n-1}(D R g+L R g)$, são limitados em $C_{\alpha^{-}, \beta^{-}}$. Pelos Lemas 3.1.8, 3.3.6 e 3.3.15, obtemos $\widetilde{H} \mathbb{P}_{n-1}(D R+L R) g \longrightarrow \widetilde{H}(D+L) R g$ uniformemente quando $n \rightarrow \infty$. Então, para $n$ suficientemente grande, obtemos

$$
\left\|\left[I+\widetilde{H} \mathbb{P}_{n-1}(D+L) R\right]-[I+\widetilde{H}(D+L) R]\right\|_{\infty, \alpha^{-}, \beta^{-}}\left\|[I+\widetilde{H}(D+L) R]^{-1}\right\|_{\infty, \alpha^{+}, \beta^{+}} \leq 1
$$

e aplicando o Lema 3.3.18, obtemos o resultado.

O teorema a seguir, nos apresenta uma estimativa para a constante de Lebesgue ponderada, que utilizamos na demonstração do teorema da convergência uniforme ponderada do MCP.

Teorema 3.3.20. ([16], Teorema 4.3.1) Sejam $f \in C_{\rho, \tau}$ e $\mathbb{P}_{n-1} f$ calculada nos zeros de $P_{n}^{\sigma, \varsigma}(x), \sigma, \varsigma>-1$. Se as condições 


$$
\frac{\sigma}{2}+\frac{1}{4} \leq \rho \leq \frac{\sigma}{2}+\frac{5}{4}, \quad \frac{\varsigma}{2}+\frac{1}{4} \leq \tau \leq \frac{\varsigma}{2}+\frac{5}{4}
$$

forem satisfeitas, então

$$
\left\|\mathbb{P}_{n-1} f\right\|_{\infty, \rho, \tau} \sim \log n
$$

onde as constantes relacionadas $a \sim$ são independentes de $n$.

Teorema 3.3.21. Sejam $a_{2} \in W_{0,0}^{r, v}, \bar{f} \in W_{\alpha^{-}, \beta^{-}}^{r^{\prime}, \eta}, R g \in W_{\alpha^{-}, \beta^{-}}^{s, q^{\prime}}$ e $L R g \in W_{\alpha^{-}, \beta^{-}}^{\widetilde{r}, \nu}$. Suponha também que $l_{x}^{\alpha^{-}, \beta^{-}} \in W_{\nu, \varsigma}^{\bar{r}, \mu}$, para $\nu, \varsigma$ constantes não negativas tais que $\nu+\alpha^{-}<1$ $e \varsigma+\beta^{-}<1$, ou se $l(x, t)=[k(x, t)-k(t, t)] /(t-x)$ seja $k_{x}^{0,0}, k_{t}^{0,0} \in W_{0,0}^{\bar{r}, \mu}$. Então para $n$ suficientemente grande

$$
\left\|g-g_{n}\right\|_{\infty, \alpha^{+}, \beta^{+}} \leq c \frac{\log ^{2} n}{n^{p}}, \quad p=\min \left\{r+v, r^{\prime}+\eta, s+q^{\prime}, \widetilde{r}+\nu, \bar{r}+\mu-\varepsilon\right\},
$$

sendo que

$$
\begin{cases}\varepsilon=0 & \text { se } l_{x}^{\rho, \tau} \in W_{\nu, \varsigma}^{\bar{r}, \mu}, \\ 0<\varepsilon \ll 1 & \text { se } \bar{r}+\mu \leq 1 \text { e } l(x, t)=[k(x, t)-k(t, t)] /(t-x) \text { e } k_{x}^{0,0}, k_{t}^{0,0} \in W_{0,0}^{\bar{r}, \mu}, \\ \varepsilon=\bar{r}+\mu-1 \quad \text { se } \bar{r}+\mu>1, \text { e } l(x, t)=[k(x, t)-k(t, t)] /(t-x) \text { e } k_{x}^{0,0}, k_{t}^{0,0} \in W_{0,0}^{\bar{r}, \mu} .\end{cases}
$$

Demonstração: Temos que

$$
\begin{aligned}
& {[I+\widetilde{H}(D+L) R] g=\widetilde{H} \bar{f}+g_{0}} \\
& {\left[I+\widetilde{H} \mathbb{P}_{n-1}\left(D+\widetilde{L}^{n}\right) R\right] g_{n}=\widetilde{H} \mathbb{P}_{n-1} \bar{f}+g_{0}}
\end{aligned}
$$

Se $g_{0}$ é determinada de forma única conforme a condição 2.9, e $[I+\widetilde{H}(D+L) R]^{-1}$ existe, pelo Lema 3.3.8, para $n$ suficientemente grande $\tilde{L}^{n} R g \longrightarrow L R g$, e portanto, no Lema 3.3.19 podemos substituir $L$ por $\widetilde{L}^{n}$, e as equações anteriores possuem solução única. Além disso, obtemos

$$
\begin{aligned}
{\left[I+\widetilde{H} \mathbb{P}_{n-1}(D R+L R)\right]\left(g-g_{n}\right) } & =\widetilde{H}\left\{\left(\bar{f}-\mathbb{P}_{n-1} \bar{f}\right)-\left(D R-\mathbb{P}_{n-1} D R\right) g-\right. \\
& \left.-\left(L R-\mathbb{P}_{n-1} L R\right) g-\mathbb{P}_{n-1}\left(L R-\widetilde{L}^{n} R\right) g_{n}\right\},
\end{aligned}
$$

e aplicando o Lema 3.3.19, obtemos

$$
\begin{aligned}
& \left\|g-g_{n}\right\|_{\infty, \alpha^{+}, \beta^{+}} \leq c\left\{\left\|\widetilde{H}\left(\bar{f}-\mathbb{P}_{n-1} \bar{f}\right)\right\|_{\infty, \alpha^{+}, \beta^{+}}+\left\|\widetilde{H}\left(D R-\mathbb{P}_{n-1} D R\right) g\right\|_{\infty, \alpha^{+}, \beta^{+}}+\right. \\
& \left.+\left\|\widetilde{H}\left(L R-\mathbb{P}_{n-1} L R\right) g\right\|_{\infty, \alpha^{+}, \beta^{+}}+\left\|\widetilde{H} \mathbb{P}_{n-1}\left(L R-\widetilde{L}^{n} R\right) g_{n}\right\|_{\infty, \alpha^{+}, \beta^{+}}\right\} .
\end{aligned}
$$


Pelo Lema 3.3.6 e pelo Corolário 3.2.3, obtemos

$$
\begin{aligned}
& \left\|g-g_{n}\right\|_{\infty, \alpha^{+}, \beta^{+}} \leq c \log n\left\|\mathbb{P}_{n-1}\right\|_{\infty, \alpha^{-}, \beta^{-}}\left\{E_{n-1}^{\alpha^{-}, \beta^{-}}(\bar{f})+E_{n-1}^{\alpha^{-}, \beta^{-}}(D R g)+E_{n-1}^{\alpha^{-}, \beta^{-}}(L R g)\right\}+ \\
& +\log (n-1)\left\|\mathbb{P}_{n-1}\left(L R g_{n}-\widetilde{L}^{n} R g_{n}\right)\right\|_{\infty, \alpha^{+}, \beta^{+}} \leq c \log n\left\|\mathbb{P}_{n-1}\right\|_{\infty, \alpha^{-}, \beta^{-}}\left\{E_{n-1}^{\alpha^{-}, \beta^{-}}(\bar{f})+\right. \\
& \left.+E_{n-1}^{\alpha^{-}, \beta^{-}}(D R g)+E_{n-1}^{\alpha^{-}, \beta^{-}}(L R g)+\left\|\left(L R-\widetilde{L}^{n} R\right) g_{n}\right\|_{\infty, \alpha^{-}, \beta^{-}}\right\} .
\end{aligned}
$$

Como $L R g \in W_{\alpha^{-}, \beta^{-}}^{\widetilde{r}, \nu}$, se $l_{x}^{\alpha^{-}, \beta^{-}} \in W_{\nu, \varsigma, t}^{\bar{r}+\mu}$ podemos concluir que $l \in C_{\alpha^{-}, \beta^{-}, x}^{\mathscr{B}} \cap C_{\nu, \varsigma, t}^{\mathscr{C}}$ para $\mathscr{B}=\left\{b_{n}\right\}, b_{n}=O\left(n^{-\widetilde{r}-\nu}\right)$, e $\mathscr{C}=\left\{c_{n}\right\}, c_{n}=O\left(n^{-\bar{r}-\mu}\right)$. Portanto, o Lema 3.3.8 pode ser aplicado. Por outro lado, se $l(x, t)=[k(x, t)-k(t, t)] /(t-x) \operatorname{com} k_{x}^{0,0}, k_{t}^{0,0} \in W_{0,0}^{\bar{r}, \mu}$, então $k \in C_{0,0, x}^{\mathscr{B}} \cap C_{0,0, t}^{\mathscr{B}}$ para $\mathscr{B}=\left\{b_{n}\right\}, b_{n}=O\left(n^{-\bar{r}-\mu}\right)$, e o Lema 3.3 .11 pode ser aplicado. Pelo teorema 3.3.20, obtemos:

$$
\begin{aligned}
& \left\|g-g_{n}\right\|_{\infty, \alpha^{+}, \beta^{+}} \leq c \log ^{2} n\left\{E_{n-1}^{\alpha^{-}, \beta^{-}}(\bar{f})+E_{n-1}^{\alpha^{-}, \beta^{-}}(D R g)+E_{n-1}^{\alpha^{-}, \beta^{-}}(L R g)\right\}+ \\
& +c \log ^{2} n\left\{\begin{array}{cll}
E_{n}^{\nu, \varsigma}\left(l_{x}^{\rho, \tau}\right) & \text { se } l \in C_{\rho, \tau, x}^{\mathscr{B}} \cap C_{\nu, \varsigma, t}, \\
n^{-(\bar{r}+\mu)} \log n & \text { se } l(x, t)=[(k(x, t)-k(t, t)] /(t-x) e \bar{r}+\mu \leq 1, \\
n^{-1} & \text { se } l(x, t)=[(k(x, t)-k(t, t)] /(t-x) e \bar{r}+\mu>1 .
\end{array}\right.
\end{aligned}
$$

Aplicando a Proposição 3.2.9 e o Lema 3.3.15, obtemos

$$
\begin{gathered}
\left\|g-g_{n}\right\|_{\infty, \alpha^{+}, \beta^{+}} \leq c \log ^{2} n\left\{\frac{1}{n^{r^{\prime}+\eta}}\left\|\bar{f}^{\left(r^{\prime}\right)} \varphi^{r^{\prime}} \omega^{\alpha^{-}, \beta^{-}}\right\|_{\infty}+\frac{1}{n^{s+q^{\prime}}}\left\|(R g)^{(s)} \varphi^{s} \omega^{\alpha^{-}, \beta^{-}}\right\|_{\infty}+\right. \\
\left.+\frac{1}{n^{r+v}}\left\|a_{2}^{(r)} \varphi^{r} \omega^{\alpha^{-}, \beta^{-}}\right\|_{\infty}+\frac{1}{n^{\widetilde{r}+\nu}}\left\|(L R g)^{\widetilde{r}} \varphi^{\widetilde{r}} \omega^{\alpha^{-}, \beta^{-}}\right\|_{\infty}\right\}+ \\
+c \log ^{2} n\left\{\begin{array}{ccc}
n^{-(\bar{r}+\mu)} & \text { se } l(x, t) \in C_{\rho, \tau, x}^{\mathscr{B}} \cap C_{\nu, \varsigma, t} \\
n^{-(\bar{r}+\mu)} \log n & \text { se } l(x, t)=[(k(x, t)-k(t, t)] /(t-x) e \bar{r}+\mu \leq 1, \\
n^{-1} & \text { se } l(x, t)=[(k(x, t)-k(t, t)] /(t-x) e \bar{r}+\mu>1 .
\end{array}\right.
\end{gathered}
$$

Então, obtemos

$$
\left\|g-g_{n}\right\|_{\infty, \alpha^{+}, \beta^{+}} \leq \frac{c}{n^{p}} \log ^{2} n, \quad p=\min \left\{r^{\prime}+\eta, s+q^{\prime}, r+v, \widetilde{r}+\nu, \bar{r}+\mu-\varepsilon\right\},
$$

com

$\begin{cases}\varepsilon=0 & \text { se } l_{x}^{\rho, \tau} \in W_{\nu, \varsigma}^{\bar{r}, \mu}, \\ 0<\varepsilon \ll 1 & \text { se } \bar{r}+\mu \leq 1 \text { e } l(x, t)=[k(x, t)-k(t, t)] /(t-x) \text { e } k_{x}^{0,0}, k_{t}^{0,0} \in W_{0,0}^{\bar{r}, \mu}, \\ \varepsilon=\bar{r}+\mu-1 & \text { se } \bar{r}+\mu>1, \text { e } l(x, t)=[k(x, t)-k(t, t)] /(t-x) \text { e } k_{x}^{0,0}, k_{t}^{0,0} \in W_{0,0}^{\bar{r}, \mu} .\end{cases}$

Deste teorema, concluímos que a convergência do MCP depende da regularidade das funções da EIDS proposta. No entanto, pelo Lema 3.3.17, p é no máximo 5/2. 


\section{Capítulo 4}

\section{Outros métodos numéricos do tipo}

\section{colocação polinomial}

4.1 Equação integro-diferencial singular analisada de outra forma

Em [4], [5] e [14], os autores investigam a equação

$$
a_{1} \varphi^{\prime}(x)+\frac{b_{1}}{\pi} \int_{-1}^{1} \frac{\varphi^{\prime}(t)}{t-x} d t+a_{2}(x) \varphi(x)+\frac{1}{\pi} \int_{-1}^{1} l(x, t) \varphi(t) d t=f(x), \quad|x|<1,
$$

com a condição de fronteira

$$
\varphi(-1)=\varphi(1)=0
$$

onde $a_{1}$ e $b_{1}$ são constantes dadas tais que $a_{1}^{2}+b_{1}^{2}=1$. As funções $a_{2}, f \in C[-1,1] \mathrm{e}$ $l \in C[-1,1]^{2}$, em [4] e [5], e $a_{2}, f \in C(-1,1)$ e $l \in C(-1,1)^{2}$, em [14], são funções dadas.

Nestes artigos, os autores aplicam a identidade

$$
\frac{d}{d x} \int_{-1}^{1} \frac{\varphi(t)}{t-x} d t=f_{-1}^{1} \frac{\varphi^{\prime}(t)}{t-x} d t-\left(\frac{\varphi(-1)}{1+x}+\frac{\varphi(1)}{1-x}\right), \quad-1<x<1,
$$

e a equação (4.1) é resolvida na forma:

$$
\frac{d}{d x}\left[a_{1} \varphi(x)+\frac{b_{1}}{\pi} \int_{-1}^{1} \frac{\varphi(t)}{t-x} d t\right]+a_{2}(x) \varphi(x)+\frac{1}{\pi} \int_{-1}^{1} l(x, t) \varphi(t) d t=f(x), \quad|x|<1,
$$

impondo-se $\varphi(-1)=\varphi(1)=0$. 
Aproximamos a função $u(x)$ por $u_{n-1}(x)=c_{0} P_{0}^{\rho, \tau}(x)+c_{1} P_{1}^{\rho, \tau}(x)+\ldots+c_{n-1} P_{n-1}^{\rho, \tau}(x)$, onde $c_{j}$ são constantes desconhecidas e definidas em [22], equação (9.1.1), por:

$$
c_{j}=\left[\frac{2^{\rho+\tau+1}}{2 j+\rho+\tau+1} \frac{\Gamma(j+\rho+1) \Gamma(j+\tau+1)}{\Gamma(j+1) \Gamma(j+\rho+\tau+1)}\right]^{-1} \int_{-1}^{1} \omega^{\rho, \tau}(x) P_{j}^{\rho, \tau}(x) u(x) d x,
$$

$j=0, \cdots, n-1$. Observe que neste caso, não podemos considerar $c_{0}=0$, pois $\int_{-1}^{1} \varphi(x) d x$ pode não ser nula. Observe também que deste ponto de vista, ao contrário do obtido no Capítulo 2, devemos ter $\rho, \tau>0$ e portanto

$$
\rho=\rho^{+}-\rho^{-} \Rightarrow \rho^{+}>\rho^{-} \text {e } \tau=\tau^{+}-\tau^{-} \Rightarrow \tau^{+}>\tau^{-}
$$

Quando substituímos $u(x)$ por $u_{n-1}(x)$ em (4.2), obtemos o resíduo

$$
\begin{aligned}
r_{n}(x) & =\sum_{j=0}^{n-1} c_{j}\left\{\frac{d}{d x}\left[a_{1} \omega^{\rho, \tau}(x) P_{j}^{\rho, \tau}(x)+\frac{b_{1}}{\pi} f_{-1}^{1} \frac{\omega^{\rho, \tau}(t) P_{j}^{\rho, \tau}(t)}{(t-x)} d t\right]+\right. \\
& \left.+a_{2}(x) \omega^{\rho, \tau}(x) P_{j}^{\rho, \tau}(x)+\int_{-1}^{1} l(x, t) \omega^{\rho, \tau}(t) P_{j}^{\rho, \tau}(t) d t\right\}-f(x) .
\end{aligned}
$$

Pelo Lema 2.2.2, a equação anterior pode ser reescrita como

$$
\begin{aligned}
r_{n}(x) & =\sum_{j=0}^{n-1} c_{j}\left\{\frac{d}{d x}\left[\frac{-2 b_{1}}{\operatorname{sen}(\pi \rho)} P_{j+1}^{-\rho,-\tau}(x)\right]+a_{2}(x) \omega^{\rho, \tau}(x) P_{j}^{\rho, \tau}(x)+\right. \\
& \left.+\int_{-1}^{1} l(x, t) \omega^{\rho, \tau}(t) P_{j}^{\rho, \tau}(t) d t\right\}-f(x)
\end{aligned}
$$

e aplicando a equação (2.19), obtemos

$$
\begin{aligned}
r_{n}(x) & =\sum_{j=0}^{n-1} c_{j}\left\{\frac{-b_{1}(j-\rho-\tau+2)}{\operatorname{sen} \pi \rho} P_{j}^{-\rho+1,-\tau+1}+a_{2}(x) \omega^{\rho, \tau}(x) P_{j}^{\rho, \tau}(x)+\right. \\
& \left.+\int_{-1}^{1} l(x, t) \omega^{\rho, \tau}(t) P_{j}^{\rho, \tau}(t) d t\right\}-f(x) .
\end{aligned}
$$

Pela unicidade da solução $\varphi(x), \alpha=\rho-1$ e $\beta=\tau-1$. Sendo assim, a quadratura do termo $\int_{-1}^{1} l(x, t) \omega^{\rho, \tau}(t) P_{j}^{\rho, \tau}(t) d t$ é calculada nos mesmos pontos de $(2.17)$.

Para que possamos obter uma estimativa da ordem de convergência do MCP aplicando o Teorema 3.3.21, devemos ter $f \in W_{\rho^{-}, \tau^{-}}^{r^{\prime}+\eta}, L R g \in W_{\rho^{-}, \tau^{-}}^{\tilde{r}+\nu} l_{x}^{\rho^{-}, \tau^{-}} \in W_{\nu, \varsigma}^{\bar{r}, \mu}$, para $\nu+\rho^{-}, \varsigma+\tau^{-}<1$ quando $l \in C_{\rho^{-}, \tau^{-}, x} \cap C_{\nu, \varsigma, t}$. Então uma restrição maior quanto a regularidade dessas funções é exigida, em comparação com a análise do Capítulo 2, pois por (4.3), $C_{\rho^{-}, \tau^{-}}$é um espaço de funções mais restrito que $C_{\alpha^{-}, \beta^{-}}$. Isto pode levar, ao aplicar o Teorema 3.3.21 na equação (4.1), a uma estimativa menor que a obtida quando 
analisamos a EIDS como no Capítulo 2, como ocorre no Exemplo 3 a seguir, onde a função $f \in W_{\rho^{-}, \tau^{-}}^{1, \eta}$, para $\rho^{-}=\tau^{-}<1 / 2$ e $\eta=1 / 2-\varepsilon, \operatorname{com} \varepsilon>0$ arbitrário.

Em [4], a equação (4.1) para $a_{1}=0$ é analisada em espaços $L^{2}$ ponderados, obtendo-se uma estimativa para a taxa de convergência dependente de restrições bastante parecidas com as apresentadas no parágrafo anterior. Em [14], a limitação dos operadores integrais é discutida em espaços $L^{1}$ ponderados, tendo restrições sobre as funções $f$ e $l$ diminuídas. Em ambos os casos, as estimativas são bastante parecidas com as apresentadas aqui.

\subsection{Convergência do método de colocação na norma uniforme}

Considere a EIDS

$$
\begin{gathered}
\frac{1}{\pi} \int_{-1}^{1} \frac{\varphi^{\prime}(t)}{t-x} d t+\int_{-1}^{1} l_{1}(x, t) \varphi^{\prime}(t) d t+a_{2}(x) \varphi(x)+\int_{-1}^{1} l_{2}(x, t) \varphi(t) d t=f(x), \\
|x|<1
\end{gathered}
$$

onde $a_{2}, f \in \mathcal{H}([-1,1])$ e $l_{1,2} \in \mathcal{H}\left([-1,1]^{2}\right)$. As condições de fronteira $\varphi(-1)=\varphi(1)=0$ também devem ser satisfeitas.

Quando aplicamos a mudança de variável

$$
u(x)=\varphi^{\prime}(x) \Rightarrow \varphi(x)=\int_{-1}^{x} u(t) d t
$$

a equação (4.4) pode ser reescrita como:

$$
\begin{gathered}
\frac{1}{\pi} \int_{-1}^{1} \frac{u(t)}{t-x} d t+\int_{-1}^{1} l_{1}(x, t) u(t) d t+a_{2}(x) \int_{-1}^{x} u(t) d t+\int_{-1}^{1} l_{2}(x, t)\left[\int_{-1}^{t} u(s) d s\right] d t= \\
=f(x),
\end{gathered}
$$

$\operatorname{com} \int_{-1}^{1} u(t) d t=0$.

De acordo com o Capítulo 2, uma solução de classe $h_{0}$ da equação (4.5), é dada por

$$
u(x)=g(x) \omega^{\alpha, \beta}(x), \quad \alpha=\beta=-1 / 2 .
$$

Em [19], é apresentada uma análise do MCP aplicado à equação (4.4), segundo a norma uniforme. Para demonstrar o teorema da convergência do MCP nesta norma, foi utilizado o teorema a seguir. 
Teorema 4.2.1. ([21], Desigualdade de Jackson) Seja $f \in C^{(s)}[-1,1]$, tal que $f^{(s)} \in \mathcal{H}^{\mu}([-1,1])$. Então,

$$
E_{n}(f)=\inf _{p_{n} \in \Pi_{n}}\left\|f-p_{n}\right\|_{\infty} \leq \frac{c}{n^{s+\mu}}
$$

Como o termo $R g(x)=\int_{-1}^{x} \omega^{\alpha, \beta}(t) g(t) d t$, é tal que $R g \notin C^{(1)}[-1,1]$, então pelo Lema 3.3.16,

$$
E_{n}(R g) \leq \frac{c}{n^{1 / 2}}
$$

Por este motivo, a análise de convergência do MCP na norma uniforme, aplicado a uma equação da forma (4.4), assegura uma taxa de conververgência de no máximo 1/2.

\subsection{Método Multhopp}

Nesta seção, apresentamos o método Multhopp conforme descrito em [13]. Este método resolve numericamente equações de Prandtl:

$$
\frac{\varphi(x)}{a_{2}(x)}-\frac{1}{2 \pi} f_{-1}^{1} \frac{\varphi^{\prime}(t)}{t-x} d t=f(x)
$$

com a condição de fronteira

$$
\varphi(-1)=\varphi(1)=0
$$

onde $a_{2}(x)$ e $f(x)$ são funções dadas em $[-1,1]$, tais que $a_{2}(x)$ não se anula, exceto possivelmente nos extremos, e $a_{2}, f \in \mathcal{H}([-1,1])$.

Fazemos a mudança de variáveis $x=\cos \vartheta$ e $t=\cos \tau$ (e definimos novamente $\varphi(\cos \vartheta)=\varphi(\vartheta))$, e a equação (4.6) é reescrita como:

$$
\frac{\varphi(\vartheta)}{a_{2}(\vartheta)}+\frac{1}{2 \pi} f_{0}^{\pi} \frac{\varphi^{\prime}(\tau)}{\cos \tau-\cos \vartheta} d \tau=f(\vartheta)
$$

Aplicamos uma interpolação de Lagrange na função $\varphi(\vartheta)$, onde os nós utilizados são os nós de Chebyshev de segunda espécie de grau $n$,

$$
x_{i}=\cos \left(\vartheta_{i}\right), \quad \vartheta_{i}=\frac{i \pi}{n+1}, 1 \leq i \leq n,
$$

e obtemos

$$
\mathbb{P}_{n-1} \varphi(x)=\frac{2}{n+1} \sum_{i=1}^{n} \varphi\left(x_{i}\right) \sum_{m=1}^{n} \operatorname{sen}\left(m \vartheta_{i}\right) \operatorname{sen}(m \vartheta)
$$


Se derivamos $\mathbb{P}_{n-1} \varphi(x)$ com respeito a $\vartheta$ e aplicamos identidades conhecidas, obtemos:

$$
\begin{aligned}
& \frac{1}{2 \pi} f_{0}^{\pi} \frac{\varphi^{\prime}(\tau)}{\cos \tau-\cos \vartheta} d \tau \simeq \frac{1}{2 \pi} f_{0}^{\pi} \frac{(\mathbb{P} \varphi)^{\prime}(\tau)}{\cos \tau-\cos \vartheta} d \tau= \\
& =\frac{1}{\pi(n+1)} \sum_{i=1}^{n} \varphi\left(x_{i}\right) \sum_{m=1}^{n} m \operatorname{sen}\left(m \vartheta_{i}\right) f_{0}^{\pi} \frac{\cos m \tau}{\cos \tau-\cos \vartheta} d \tau= \\
& =\frac{1}{n+1} \sum_{i=1}^{n} \varphi\left(x_{i}\right) \sum_{m=1}^{n} \frac{m \operatorname{sen}\left(m \vartheta_{i}\right) \operatorname{sen}(m \vartheta)}{\operatorname{sen} \vartheta}
\end{aligned}
$$

Ao substituirmos $\varphi(x)$ por $\mathbb{P}_{n-1} \varphi(x)$ em (4.7), considerando $\vartheta=\vartheta_{j}, j=1, \cdots, n$, e aplicando as expressões (4.8) e (4.9), obtemos o sistema de equações a ser resolvido $A \varphi=f$, onde

$$
\begin{aligned}
& A_{i, j}=\frac{1}{n+1}\left[\sum_{m=1}^{n} \operatorname{sen}\left(m \vartheta_{i}\right) \operatorname{sen}\left(m \vartheta_{j}\right)\left(\frac{2}{a_{2}\left(\vartheta_{j}\right)}+\frac{m}{\operatorname{sen} \vartheta_{j}}\right)\right], \\
& \varphi=\left[\varphi\left(\vartheta_{1}\right) \cdots \varphi\left(\vartheta_{n}\right)\right]^{\top} \text { e } f=\left[f\left(\vartheta_{1}\right) \cdots f\left(\vartheta_{n}\right)\right]^{\top}, 1 \leq j \leq n .
\end{aligned}
$$

A solução do sistema (4.10) é aplicada na expressão (4.8), que aproxima a solução de (4.7).

O método Multhopp pode ser estendido para EIS da forma:

$$
\frac{1}{2 \pi} \int_{-1}^{1} \frac{\varphi(t)}{t-x} d t+\frac{1}{2 \pi} \int_{-1}^{1} l(x, t) \varphi(t) d t=f(x),
$$

para $\varphi(x)=\varphi_{0}(x)(1-x)^{\rho}(1+x)^{\tau} \operatorname{com} \rho=\tau= \pm 1 / 2$ ou $\rho=-\tau= \pm 1 / 2$, e $l(x, t)$ contínua em $[-1,1]^{2}$. Para o caso $\rho=\tau=1 / 2$, através de uma quadratura de Gauss-Chebyshev obtemos:

$$
\frac{1}{2 \pi} \int_{-1}^{1} l(x, t) \varphi(t) d t \simeq \frac{1}{2(n+1)} \sum_{i=1}^{n} \operatorname{sen} \vartheta_{i} l\left(\cos \vartheta, \cos \vartheta_{i}\right) \varphi_{0}\left(\vartheta_{i}\right)
$$

Nos exemplos do Capítulo 5 em que $a_{1}=0$ e $\varphi(-1)=\varphi(1)=0$, aplicamos o método Multhopp adaptado para equações da forma (4.1), onde os termos

$$
a_{2}(x) \varphi(x)-\frac{1}{\pi} \int_{-1}^{1} \frac{\varphi^{\prime}(t)}{t-x} d t \text { e } \frac{1}{\pi} \int_{-1}^{1} l(x, t) \varphi(t) d t
$$

são calculados de forma análoga à (4.10) e (4.12) respectivamente. Nos casos em que $a_{1} \neq 0$, não é possível aplicar o método Multhopp, sendo esta a desvantagem deste método em relação ao $\mathrm{MCP}$, pois como veremos a seguir, o método de Colocação é tão eficiente quanto o método Multhopp. 


\section{Capítulo 5}

\section{Exemplos Numéricos}

Nesta seção, apresentaremos alguns exemplos numéricos que confirmam os resultados teóricos obtidos na seção anterior.

O MCP provém da aproximação de $g(x)$ pelo polinômio $g_{n}(x)$, conforme definido na equação (2.10). Sendo assim, a aproximação de $\varphi(x)$ é dada por

$$
\varphi_{n}(x)=\int_{-1}^{x} g_{n}(t) \omega^{\alpha, \beta}(t) d t=\sum_{j=1}^{n} c_{j} \int_{-1}^{x} P_{j}^{\alpha, \beta}(t) \omega^{\alpha, \beta}(t) d t
$$

e pela identidade (2.13), obtemos

$$
\varphi_{n}(x)=-\sum_{j=1}^{n} \frac{c_{j}}{2 j} P_{j-1}^{\alpha+1, \beta+1}(x) \omega^{\alpha+1, \beta+1}(x) .
$$

Lembramos que pela Observação 3.2.8, se $g \in C_{\alpha^{+}, \beta^{+}}$então $\varphi \in C_{0,0}$, e apenas por conveniência nas demostrações sobre a convergência do MCP aproveitamos o fato de $C_{0,0} \subset C_{\alpha^{-}, \beta^{-}}$. Por este motivo, os resultados numéricos serão indicados por $e_{n}=\left\|\varphi-\varphi_{n}\right\|_{\infty}$.

Os dois primeiros exemplos que tratamos aqui, foram propostos em [19].

\section{Exemplo 1}

Considere a equação

$$
\varphi(x)+\frac{1}{\pi} \int_{-1}^{1} \frac{\varphi^{\prime}(t)}{t-x} d t+\int_{-1}^{1} \frac{\cos x}{1+(x t)^{2}} \varphi(t) d t=\varphi(x)+\frac{2 x}{\pi \sqrt{1-x^{2}}} \log \left|\frac{\sqrt{1-x^{2}}-x+1}{\sqrt{1-x^{2}}+x-1}\right|,
$$

com a condição de fronteira $\varphi(-1)=\varphi(1)=0$. 
A solução analítica desta equação é

$$
\varphi(x)= \begin{cases}\sqrt{1-x^{2}}+\frac{2}{\pi}(\arccos (x)-\pi) & \text { se } x \leq 0 \\ \frac{2}{\pi} \arccos (x)-\sqrt{1-x^{2}} & \text { se } x \geq 0\end{cases}
$$

$\operatorname{com} \varphi^{\prime}(x)=(|x|-2 / \pi) / \sqrt{1-x^{2}}$, isto é $\omega^{\alpha, \beta}(x)=1 / \sqrt{1-x^{2}}$ e $g(x)=|x|-2 / \pi$.

Como $l(x, t)=\cos x /\left[1+(x t)^{2}\right]$, então $l \in C_{0,0, x} \cap C_{0,0, t}, L R g \in W_{1 / 2,1 / 2}^{\infty} \mathrm{e}$ $l_{x}^{1 / 2,1 / 2} \in W_{0,0}^{\infty}$. A função $a_{2} \in W_{1 / 2,1 / 2}^{\infty}$ e $f \in C_{1 / 2,1 / 2}$, sendo que

$$
f^{\prime}(x)=\frac{1}{\sqrt{1-x^{2}}}\left\{s x-\frac{2}{\pi}\left[1+\frac{1}{\sqrt{1-x^{2}}}-2 \log \left(\frac{1-x+\sqrt{1-x^{2}}}{x-1+\sqrt{1-x^{2}}}\right) /\left(1-x^{2}\right)\right]\right\}
$$

onde $s=-1$ se $x \leq 0$ e $s=1$ se $x \geq 0$. Portanto, $f \in W_{1 / 2,1 / 2}^{0,1 / 2}$ e pelo Lema 3.3.17, $R g \in W_{1 / 2,1 / 2}^{1,1 / 2}$. Então, pelo Teorema 3.3.21 a estimativa para o erro é $\log ^{2} n / n^{1 / 2}$.

\begin{tabular}{|c|ccccccc|}
\hline$n$ & 2 & 4 & 8 & 16 & 32 & 64 \\
\hline \hline$e_{n}^{(1)}$ & 0.03747 & 0.018804 & 0.005686 & 0.001399 & 0.000341 & 0.000340 \\
$\tau^{(1)}$ & 0.994808 & 1.725474 & 2.022615 & 2.039638 & 2.025002 \\
\hline$e_{n}^{(2)}$ & 0.051386 & 0.016672 & 0.004782 & 0.001258 & 0.000321 & 0.000081 \\
$\tau^{(2)}$ & \multicolumn{1}{|c}{1.623958} & 1.801683 & 1.926198 & 1.969541 & 1.985031 \\
\hline
\end{tabular}

Tabela 5.1: (1)-MCP; (2)-Método Multhopp.

A Tabela 5.1 mostra uma aproximação da taxa de convergência para os métodos de Colocação e Multhopp, calculada da forma $\tau=\log \left(\frac{e_{n}}{e_{2 n}}\right) / \log 2$.

Os resultados obtidos neste exemplo mostram que o MCP é tão eficiente quanto o método Multhopp, em se tratando de aproximar numericamente equações do tipo Prandtl's, com a vantagem de ser mais geral. Além disso, a Tabela 5.1 mostra uma taxa de convergência de aproximadamente 2, melhor que a estimativa que obtivemos aplicando o Teorema 3.3.21. Esta variação se deve à limitação do Teorema 3.3.21 para obter uma estimativa mais próxima da observada na prática em certos casos.

No próximo exemplo, mostramos como as estimativas dependem fortemente da regularidade de $l(x, t)$ e de $f(x)$, propondo $l(x, t)$ não regular em ambas as variáveis o que leva à não regularidade de $f(x)$, depois regular apenas em relação a $x$, levando a regularidade de $f(x)$ também, e finalmente regular. 


\section{Exemplo 2}

Considere a equação

$$
\sqrt{1-x^{2}} \varphi(x)-\frac{1}{\pi} \int_{-1}^{1} \frac{\varphi^{\prime}(t)}{t-x} d t+\frac{1}{\pi} \int_{-1}^{1}(|x|+|t|) \varphi(t) d t=2-x^{2}+\frac{|x|}{2}+\frac{2}{3 \pi}
$$

com a condição de fronteira $\varphi(-1)=\varphi(1)=0$.

A solução analítica desta equação é $\varphi(x)=\sqrt{1-x^{2}}$, sendo então $g(x)=-x$ e $\omega^{\alpha, \beta}(x)=1 / \sqrt{1-x^{2}}$.

Como $l(x, t) \in C_{0,0, x} \cap C_{0,0, t}$, obtemos $L R g \in W_{1 / 2,1 / 2}^{0,1 / 2}$ e $l_{x}^{1 / 2,1 / 2} \in W_{0,0}^{0,1}$. Já $a_{2}(x)=\sqrt{1-x^{2}}$, então $a_{2} \in W_{1 / 2,1 / 2}^{2,1}$ e $f(x) \in W_{1 / 2,1 / 2}^{0,1 / 2}$. Portanto, $R g \in W_{1 / 2,1 / 2}^{1,1 / 2}$ e a estimativa para o erro assintótico é $\log ^{2} n / \sqrt{n}$. Veja os resultados:

\begin{tabular}{|c|cccccccc|}
\hline$n$ & 2 & 4 & & 8 & & 16 & 32 & 64 \\
\hline \hline$e_{n}^{(1)}$ & 0.017966 & 0.004940 & 0.001477 & 0.000409 & 0.000108 & 0.000028 \\
$\tau^{(1)}$ & \multicolumn{2}{|c|}{1.8626} & 1.7423 & 1.8521 & 1.9211 & 1.9475 \\
\hline$e_{n}^{(2)}$ & 0.016797 & 0.004996 & 0.001479 & 0.000409 & 0.000108 & 0.000028 \\
$\tau^{(2)}$ & \multicolumn{2}{|c|}{1.7494} & 1.7562 & 1.8544 & 1.9211 & 1.9475 \\
\hline
\end{tabular}

Tabela 5.2: (1)-MCP; (2)-Método Multhopp.

Neste caso, a solução $g(x)$ é um polinômio de primeiro grau e era de se esperar que a convergência dos métodos fossem imediatas, o que não ocorreu. Isto porque, outras funções envolvidas nos cálculos, $f$ e $l$, não são suaves.

Se na mesma equação escolhermos $l(x, t)=x+|t|$, então $L R g \in W_{1 / 2,1 / 2}^{\infty} \mathrm{e}$ $l_{x}^{1 / 2,1 / 2} \in W_{0,0}^{0,1}$ como anteriormente. Agora $f(x)=2-x^{2}+x / 2+2 /(3 \pi)$, então $f \in W_{1 / 2,1 / 2}^{\infty}$ e pelo Lema 3.3.17, $R g \in W_{1 / 2,1 / 2}^{2,1 / 2}$. Então a estimativa para o erro é $\log ^{2} n / n$.

\begin{tabular}{|c|cccccccc|}
\hline$n$ & 2 & 4 & 8 & & 16 & 32 & 64 \\
\hline \hline$e_{n}^{(1)}$ & 0.022009 & 0.005580 & 0.001632 & 0.000450 & 0.000119 & 0.000030 \\
$\tau^{(1)}$ & \multicolumn{2}{|c}{1.9798} & 1.7738 & 1.8598 & 1.91908 & 1.9406 \\
\hline$e_{n}^{(2)}$ & 0.019197 & 0.005593 & 0.001632 & 0.000450 & 0.000119 & 0.000030 \\
$\tau^{(2)}$ & \multicolumn{2}{|c}{1.7792} & 1.7770 & 1.8586 & 1.9190 & 1.9406 \\
\hline
\end{tabular}

Tabela 5.3: (1)-MCP; (2)-Método Multhopp. 
Quando consideramos $l(x, t)=|x|+t$, obtemos $f(x)=2-x^{2}+|x| / 2$. Então $L R g \in W_{1 / 2,1 / 2}^{0,1 / 2}, l_{x}^{1 / 2,1 / 2} \in W_{0,0}^{\infty}, f \in W_{1 / 2,1 / 2}^{0,1 / 2}$ e $R g \in W_{1 / 2,1 / 2}^{1,1 / 2}$. A estimativa para o erro é $\log ^{2} n / n^{1 / 2}$.

Mas se consideramos $l(x, t)=x+t$, obtemos $f(x)=2-x^{2}+x / 2$ e portanto, $l_{x}^{1 / 2,1 / 2} \in W_{0,0}^{\infty}$ e $f \in W_{1 / 2,1 / 2}^{\infty}$. Então $R g \in W_{1 / 2,1 / 2}^{2,1 / 2}$ e a estimativa para o erro é $\log ^{2} n / n^{5 / 2}$. Na prática, ambos os métodos produziram a solução exata para $l(x, t)=|x|+t$ e $l(x, t)=x+t$

Como observamos na Tabela 5.3, mesmo com a suavidade das funções $f(x)$ e $l(x, t)$ (em relação a $x$ ), os resultados foram similares aos do caso anterior. Isto mostra o quanto a irregularidade em relação à variável de integração influencia no resultado.

O próximo exemplo mostra porque é mais vantajoso representar a EIDS como descrito na Seção 2.1, e não da forma descrita na Seção 4.1.

\section{Exemplo 3}

Considere a equação

$$
\frac{1}{\pi} \int_{-1}^{1} \frac{\varphi^{\prime}(t)}{t-x} d t+\varphi(x)+\int_{-1}^{1} x|t| \varphi(t) d t=-1+\frac{2}{3} x+\sqrt{1-x^{2}}
$$

com a condição de fronteira $\varphi(-1)=\varphi(1)=0$.

A solução analítica desta equação é $\varphi(x)=\sqrt{1-x^{2}}$. Portanto, $\omega^{\alpha, \beta}(x)=1 / \sqrt{1-x^{2}}$ e $g(x)=-x$.

A função $L R g \in W_{1 / 2,1 / 2}^{\infty}$, e $l_{x}^{1 / 2,1 / 2} \in W_{0,0}^{0,1}$. Em relação à regularidade de $f(x)$, temos:

$$
f^{\prime}(x)=\frac{2}{3}-\frac{x}{\sqrt{1-x^{2}}}, \quad f^{\prime \prime}(x)=-\frac{1}{\left(1-x^{2}\right)^{3 / 2}} \quad \text { e } \quad f^{\prime \prime \prime}(x)=-\frac{3 x}{\left(1-x^{2}\right)^{5 / 2}} .
$$

Portanto $f \in W_{1 / 2,1 / 2}^{2,1}, R g \in W_{1 / 2,1 / 2}^{2,1 / 2}$, e a estimativa para o erro é $\log ^{2} n / n$. 


\begin{tabular}{|c|cccccccc|}
\hline$n$ & 2 & 4 & 8 & & 16 & 32 & 64 \\
\hline \hline$e_{n}^{(1)}$ & 0.045917 & 0.013604 & 0.004015 & 0.001104 & 0.000292 & 0.000075 \\
$\tau^{(1)}$ & \multicolumn{1}{|c|}{1.7550} & 1.7606 & 1.8627 & 1.9187 & 1.9610 & \\
\hline$e_{n}^{(2)}$ & 0.052352 & 0.013811 & 0.004015 & 0.001104 & 0.000292 & 0.000075 \\
$\tau^{(2)}$ & \multicolumn{2}{|c|}{1.9224} & 1.7823 & 1.8627 & 1.9187 & 1.9610 \\
\hline
\end{tabular}

Tabela 5.4: (1)-MCP; (2)-Método Multhopp.

Assim como no Exemplo 2, a irregularidade de $l$ em relação a $t$ limitou a estimativa do erro a $1-\varepsilon, \varepsilon>0$, embora na prática ambos os métodos tenham convergência de ordem aproximadamente 2 .

Quando substituímos $l(x, t)=x|t|$ por $l(x, t)=x t$, obtemos $f(x)=-1+\sqrt{1-x^{2}} \mathrm{e}$

$$
f^{\prime}(x)=-\frac{x}{\sqrt{1-x^{2}}}, \quad f^{\prime \prime}(x)=-\frac{1}{\left(1-x^{2}\right)^{3 / 2}} \quad \text { e } \quad f^{\prime \prime \prime}(x)=-\frac{3 x}{\left(1-x^{2}\right)^{5 / 2}} .
$$

Portanto, $l_{x}^{1 / 2,1 / 2} \in W_{0,0}^{\infty}, f \in W_{1 / 2,1 / 2}^{2,1}$ e $R g \in W_{1 / 2,1 / 2}^{2,1 / 2}$. Então, a estimativa para o erro é $\log ^{2} n / n^{5 / 2}$. Na prática, ambos os métodos produziram a solução exata para este caso.

Observe que neste útimo caso, se $\alpha^{-}$e $\beta^{-}$fossem menores que $1 / 2$, obteríamos $f \in W_{\alpha^{-}, \beta^{-}}^{1, q}$ para $q=\min \left\{\alpha^{-}, \beta^{-}\right\}$o que mudaria a estimativa do erro para $\log ^{2} n / n^{1+q}$, bem menor que a obtida por nós. A estimativa para o erro $\log ^{2} n / n^{1+q}$, é obtida quando analisamos a equação (5.2) como na Seção 4.1.

O próximo exemplo, propõe a resolução de uma equação para a qual $\alpha \neq \beta$. Conforme descrito na Seção 4.3, este é um dos casos em que o método Multhopp não se aplica.

\section{Exemplo 4}

Considere a equação

$$
\begin{aligned}
& \frac{1}{\sqrt{2}} \varphi^{\prime}(x)-\frac{1}{\sqrt{2} \pi} \int_{-1}^{1} \frac{\varphi^{\prime}(t)}{t-x} d t+\varphi(x)+\int_{-1}^{1}|x t| \varphi(t) d t= \\
& =\frac{1}{48}|x|\left[-14-3 \sqrt{2} \log \left(\cot \frac{\pi}{8}\right)\right]-\frac{1}{2}-\frac{1}{2}(1-x)^{1 / 4}(1+x)^{3 / 4}
\end{aligned}
$$

com a condição de fronteira $\varphi(-1)=\varphi(1)=0$.

A solução analítica desta equação é $\varphi(x)=-(1-x)^{1 / 4}(1+x)^{3 / 4} / 2$. Portanto, $g(x)=(2 x-1) / 4$ e $\omega^{\alpha, \beta}(x)=(1-x)^{-3 / 4}(1+x)^{-1 / 4}$. 
Neste caso, $L R g \in W_{3 / 4,1 / 4}^{0,1 / 4}$ e $l_{x}^{3 / 4,1 / 4} \in W_{0,0}^{0,1}$. A função $f \in W_{3 / 4,1 / 4}^{0,1 / 4}$ e portanto, $R g \in W_{3 / 4,1 / 4}^{1,1 / 4}$. Então a estimativa para o erro assintótico é $\log n^{2} / n^{1 / 4}$. Veja os resultados obtidos:

\begin{tabular}{|c|rrrrrr|}
\hline$n$ & 2 & 4 & 8 & 16 & 32 \\
\hline$e_{n}$ & 0.018785 & 0.003684 & 0.001011 & 0.000270 & 0.00070 \\
$\tau$ & \multicolumn{2}{|c}{2.3502} & 1.8655 & 1.9048 & 1.9475 & \\
\hline
\end{tabular}

Tabela 5.5: MCP.

Se na mesma equação escolhemos $l(x, t)=x|t|$, então $L R g \in W_{3 / 4,1 / 4}^{\infty} \mathrm{e}$ $f(x)=\frac{1}{48} x\left[-14-3 \sqrt{2} \log \left(\cot \frac{\pi}{8}\right)\right]-\frac{1}{2}-\frac{1}{2}(1-x)^{1 / 4}(1+x)^{3 / 4}$,

$f^{\prime}(x)=-\frac{14}{48}-\frac{3 \sqrt{2}}{48} \log \left(\cot \frac{\pi}{8}\right)-\frac{18}{48}(1-x)^{1 / 4}(1+x)^{-1 / 4}+\frac{6}{48}(1-x)^{-3 / 4}(1+x)^{3 / 4}$,

$f^{\prime \prime}(x)=\frac{3}{8}(1-x)^{-7 / 4}(1+x)^{-5 / 4}$,

$f^{\prime \prime \prime}(x)=\frac{3+18 x}{16}(1-x)^{-11 / 4}(1+x)^{-9 / 4}$.

Portanto, $f \in W_{3 / 4,1 / 4}^{2,1}$ e $R g \in W_{3 / 4,1 / 4}^{2,1 / 4}$. No entanto, como $l_{x}^{3 / 4,1 / 4} \in W_{0,0}^{0,1}$, a estimativa para o erro é $\log n^{2} / n$. Os resultados obtidos foram:

\begin{tabular}{|c|cccccc|}
\hline$n$ & 2 & 4 & 8 & 16 & 32 \\
\hline$e_{n}$ & 0.013781 & 0.003685 & 0.001014 & 0.000272 & 0.000071 \\
$\tau$ & \multicolumn{2}{|c|}{1.9284} & 1.8275 & 1.8970 & 1.9432 & \\
\hline
\end{tabular}

Tabela 5.6: MCP.

Quando consideramos $l(x, t)=x t$, o método produz a solução exata.

Este exemplo mostra que para $\alpha \neq \beta$, os resultados obtidos são análogos aos dos Exemplos 2 e 3 , indicando novamente, que a não regularidade de $l(x, t)$ influencia fortemente na convergência do método.

O próximo exemplo, mostra o comportamento das soluções $\varphi(x)$, tais que $\varphi^{\prime}(x)=g(x) \omega^{\alpha, \beta}(x)$, quando $\alpha$ e $\beta$ são próximos de 0 e -1 , respectivamente. Lembramos novamente que neste caso o método Multhopp não se aplica. 


\section{Exemplo 5}

Considere a equação

$$
\begin{aligned}
& \frac{1}{4}(1-\sqrt{5}) \varphi^{\prime}(x)-\frac{1}{\pi} \sqrt{\frac{5}{8}+\frac{\sqrt{5}}{8}} \int_{-1}^{1} \frac{\varphi^{\prime}(t)}{t-x} d t+\varphi(x)+\int_{-1}^{1}(|x|+|t|) \varphi(t) d t= \\
& =-1.9718-0.914977|x|-\frac{1}{2}(1-x)^{9 / 10}(1+x)^{1 / 10},
\end{aligned}
$$

com a condição de fronteira $\varphi(-1)=\varphi(1)=0$.

A solução analítica desta equação é $\varphi(x)=-\frac{1}{2}(1-x)^{9 / 10}(1+x)^{1 / 10}$, o que implica em $g(x)=\frac{5 x+4}{10}$ e $\omega^{\alpha, \beta}(x)=(1-x)^{-1 / 10}(1+x)^{-9 / 10}$.

Neste caso, $L R g \in W_{1 / 10,9 / 10}^{0,1 / 10}, l_{x}^{1 / 10,9 / 10} \in W_{0,0}^{0,1}$ e $f \in W_{1 / 10,9 / 10}^{0,1 / 10}$, sendo a estimativa para o erro $\log ^{2} n / n^{1 / 10}$.

\begin{tabular}{|c|cccccc|}
\hline$n$ & 2 & 4 & 8 & 16 & 32 \\
\hline$e_{n}$ & 0.011393 & 0.003207 & 0.000830 & 0.000209 & 0.000052 \\
$\tau$ & & 1.8289 & 1.9500 & 1.98960 & 2.0069 \\
\hline
\end{tabular}

Tabela 5.7: MCP.

Se na mesma equação escolhermos $l(x, t)=x+|t|$, então $L R g \in W_{1 / 10,9 / 10}^{\infty} \mathrm{e}$

$$
\begin{aligned}
f(x)= & -1.9718-\frac{1}{2}(1-x)^{9 / 10}(1+x)^{1 / 10}-0.914977 x \\
f^{\prime}(x)= & -0.914977+0.45(1-x)^{-1 / 10}(1+x)^{1 / 10}-0.05(1-x)^{9 / 10}(1+x)^{-9 / 10}, \\
f^{\prime \prime}(x)= & 0.09(1-x)^{-1 / 10}(1+x)^{-9 / 10}+0.045\left[(1-x)^{9 / 10}(1+x)^{-19 / 10}+\right. \\
& \left.+(1-x)^{-11 / 10}(1+x)^{1 / 10}\right], \\
f^{\prime \prime \prime}(x)= & -0.1215(1-x)^{-1 / 10}(1+x)^{-19 / 10}+0.0135(1-x)^{-11 / 10}(1+x)^{-9 / 10}- \\
& -0.0855(1-x)^{9 / 10}(1+x)^{-29 / 10}+0.0495(1-x)^{1 / 10}(1+x)^{-21 / 10} .
\end{aligned}
$$

Portanto, $f \in W_{1 / 10,9 / 10}^{2,1}$ e $R g \in W_{1 / 10,9 / 10}^{2,1 / 10}$. Mas a estimativa para o erro é $\log ^{2} n / n$, pois $l_{x}^{1 / 10,9 / 10} \in W_{0,0}^{0,1}$.

Quando consideramos $l(x, t)=x+t$, o método produz a solução exata.

Observamos que mesmo para valores de $\alpha$ e $\beta$ próximos a 0 e -1 respectivamente, o método mostrou resultados análogos aos obtidos nos Exemplos 2, 3 e 4, conforme o esperado. 


\begin{tabular}{|c|cccccc|}
\hline$n$ & 2 & 4 & 8 & 16 & 32 \\
\hline$e_{n}$ & 0.0150803 & 0.003959 & 0.001015 & 0.000256 & 0.000064 \\
$\tau$ & \multicolumn{2}{|c}{1.9295} & 1.9637 & 1.9873 & 2.0000 \\
\hline
\end{tabular}

Tabela 5.8: MCP.

O próximo exemplo, proposto em [13], Seção 3.2, apresenta uma equação que rege o estado de tensão em um corpo elástico, quando um disco rígido de base plana é inserido em um orifício circular de mesmo raio em um meio elástico plano infinito, pressionado por uma força ao longo do eixo do orifício. É considerado que a tensão e a rotação se anulam no infinito.

\section{Exemplo 6 - Disco rígido circular}

Considere a equação

$$
\begin{gathered}
-\frac{1}{2 \pi} \int_{-1}^{1} \frac{N^{\prime}(t)}{t-x} d t+\frac{\chi-1}{\chi+1} \frac{\beta}{x^{2}+\beta^{2}} N(x)-\frac{\beta^{2}}{\pi\left(x^{2}+\beta^{2}\right)} \int_{-1}^{1} \frac{N(t)}{t^{2}+\beta^{2}} d t= \\
=\frac{2 \chi \beta}{\chi+1} \frac{x^{2}-\beta^{2}}{\left(x^{2}+\beta^{2}\right)^{2}}, \quad N(-1)=N(1)=0
\end{gathered}
$$

$\operatorname{com} \beta=1.20886$ e $\chi=5 / 3$. A constante $N$ representa a componente normal da força de tensão externa agindo no centro do disco em relação ao deslocamento do disco no meio elástico, $\chi$ representa a constante elástica do material em que o disco é inserido, e $\beta$ está associado à região de contato entre o disco e a força aplicada sobre ele.

Por considerar que o disco é tensionado em um plano elástico infinito, por motivos teóricos (descritos em [13]), a condição

$$
c(\beta)=\frac{2 \beta}{\pi} \int_{-1}^{1} \frac{t^{2}-\beta^{2}}{\left(t^{2}+\beta^{2}\right)^{2}} N(t) d t=1
$$

é imposta.

A Tabela 5.9, apresenta a solução numérica da equação com determinados valores de $x$ para $n=7$ e $n=150$. O termo $c(\beta)$ foi aproximado por uma quadratura de Gauss-Chebyshev de segunda espécie. 


\begin{tabular}{|c||c|c|c|c||c|}
\hline$x$ & 0 & 0.3827 & 0.7071 & 0.9239 & $c$ \\
\hline$N_{7}^{(1)}$ & -1.9173 & -1.6560 & -1.1084 & -0.5436 & 0.999534 \\
\hline$N_{7}^{(2)}$ & -1.9162 & -1.6555 & -1.1090 & -0.5425 & 0.999324 \\
\hline$N_{150}^{(1)}$ & -1.9167 & -1.6556 & -1.1090 & -0.5426 & 0.999324 \\
\hline$N_{150}^{(2)}$ & -1.9167 & -1.6556 & -1.1090 & -0.5426 & 0.999324 \\
\hline
\end{tabular}

Tabela 5.9: (1)-MCP; (2)-Método Multhopp.

Observe que a equação (5.3) não está na forma de Prandtl's, para a qual o método Multhopp é aplicado. Mas este, pôde ser estendido aproximando o termo $\int_{-1}^{1} N(t) /\left(t^{2}+\beta^{2}\right) d t$ por uma quadratura de Gauss-Chebyshev de segunda espécie.

O próximo exemplo, proposto em [24], apresenta a equação que analisa o comportamento de velas de barco sob a ação de forças hidro e aero-dinâmicas, geradas por um escoamento não viscoso incompressível. A forma efetiva com que o escoamento afeta a tridimensionalidade da vela, está associada à geometria e resistência da vela. O ângulo de incidência entre a corda da vela e o escoamento do fluido, são aspectos levados em cosideração na modelagem do problema.

\section{Exemplo 7 - Equação da Vela}

Considere a equação

$$
\frac{1}{\pi} \int_{0}^{1} \frac{S^{\prime \prime}(t)}{t-x} d t-\lambda S^{\prime}(x)=\lambda \gamma
$$

com as condições de fronteira $S(0)=S(1)=0$ e $S^{\prime \prime}(1)=0$. O coeficiente $\gamma$ denota o ângulo entre a vela e o escoamento do fluido, o parâmetro $\lambda$ é uma constante inversamente proporcional à tensão na vela, e a solução $S$ é a ordenada da vela associada a $x$.

A partir de uma mudança de variável, podemos reescrever a equação (5.4) como

$$
\frac{1}{\pi} \int_{0}^{1} \frac{\varphi^{\prime}(t)}{t-x} d t-\lambda \varphi(x)=\lambda \gamma
$$

com as seguintes condições de fronteira: $\int_{0}^{1} \varphi(t) d t=0$ e $\varphi^{\prime}(1)=0$. Com a nova mudança de variável $\varphi^{\prime}(x)=u(x)$, obtemos

$$
\frac{1}{\pi} f_{0}^{1} \frac{u(t)}{t-x} d t+\lambda\left(\int_{0}^{x} u(t) d t-\int_{0}^{1} \int_{0}^{t} u(s) d s d t\right)=\lambda \gamma
$$


com a condição de fronteira $u(1)=0$. Para reescrer a equação $(5.4)$ no intervalo $[-1,1]$, aplicamos outra mudança de variável, donde obtemos

$$
\frac{1}{\pi} \int_{-1}^{1} \frac{\tilde{u}(t)}{t-x} d t+\frac{\lambda}{2}\left(\int_{-1}^{x} \tilde{u}(t) d t-\frac{1}{2} \int_{-1}^{1} \int_{-1}^{t} \tilde{u}(s) d s d t\right)=\lambda \gamma,
$$

com a condição de fronteira $\tilde{u}(1)=0$.

A condição de fronteira no extremo $x=1$, implica que o índice do problema é $\varkappa=0$ e

$$
\tilde{u}(x)=(1-x)^{1 / 2}(1+x)^{-1 / 2} g(x) .
$$

Toda a teoria desenvolvida anteriormente é válida, exceto pela não necessidade de condições adicionais.

Em [24], o autor resolveu a equação (5.5) por um método de aproximação baseado na transformação dessa equação utilizando fórmulas trigonométricas. Resolvemos a equação (5.5), considerando $n=21$ e os pontos de colocação $x(i)=-\cos \left(\theta_{i}\right)$, onde $\theta_{i}=(i \pi) /(n-1), \quad i=0, \cdots, n-1$. A partir desta solução, obtemos a solução da equação equivalente (5.4) nos pontos $x(i)=\frac{1}{2}\left(1-\cos \left(\theta_{i}\right)\right)$. Consideramos $\gamma=\pi / 18$ e $\lambda=5.507$, pois este é um dos autovalores da matriz associada ao sistema obtido para resolver a equação (5.5) pelo método de [24], o que indica soluções com ângulos de incidência críticos, o que propicia uma mudança qualitativa no comportamento da solução.

\begin{tabular}{|c|c|l|c|c|l|}
\hline$x_{i}$ & $S^{(1)} / \sqrt{0.073}$ & $S^{(2)} / \sqrt{0.073}$ & $x_{i}$ & $S^{(1)} / \sqrt{0.073}$ & $S^{(2)} / \sqrt{0.073}$ \\
\hline 0 & 0.000000 & -0.000000 & 0.5000 & -0.000933 & -0.000923 \\
0.0062 & 0.013530 & 0.013526 & 0.5782 & -0.144977 & -0.144966 \\
0.0245 & 0.053225 & 0.053221 & 0.6545 & -0.255501 & -0.255492 \\
0.0545 & 0.115068 & 0.115067 & 0.7270 & -0.311230 & -0.311224 \\
0.0955 & 0.189678 & 0.189674 & 0.7939 & -0.309007 & -0.309002 \\
0.1464 & 0.261087 & 0.261090 & 0.8536 & -0.261570 & -0.261570 \\
0.2061 & 0.308207 & 0.308207 & 0.9045 & -0.189926 & -0.189924 \\
0.2730 & 0.310063 & 0.3100717 & 0.9455 & -0.115171 & -0.115175 \\
0.3455 & 0.253984 & 0.2539897 & 0.9755 & -0.053254 & -0.532535 \\
0.4218 & 0.143206 & 0.143218 & 0.9938 & -0.013534 & -0.135392 \\
& & & 1.0000 & 0.000000 & 0.000000 \\
\hline
\end{tabular}

Tabela 5.10: (1)-MCP; (2)-Método de [24]. 
Por conveniência, o autor em [24] apresentou os resultados sob a forma $S / \sqrt{0.073}$, onde $\sqrt{0.073}$ é um valor associado a um pequeno ângulo crítico de incidência. 


\section{Capítulo 6}

\section{Conclusões}

O objetivo desta tese, é apresentar uma estimativa para a taxa de convergência do MCP, usado para resolver equações integro-diferenciais singulares com núcleo de Cauchy.

Os pontos de colocação usados são os zeros do $n$-ésimo polinômio de Chebyshev de primeira espécie, pois conforme o Teorema 3.3.20, neste caso temos uma estimativa para a constante de Lebesgue ponderada, necessária para estimarmos a ordem de convergência do método. Nos casos em que se faz necessário, aplicamos uma regra de quadratura de Gauss-Jacobi com $n$ nós para aproximar a integral $L g_{n}$. Foram consideradas condições de fronteira não nulas, que devido à mudanças de variáveis necessárias às resolução da EIDS, geram novas irregularidades nos dados da equação a ser resolvida.

Apresentamos a análise de convergência do MCP segundo uma norma uniforme ponderada, além de uma estimativa para a taxa de convergência do método, que depende da regularidade das funções envolvidas. Esta análise mostra que a estimativa para a taxa de convergência do método, pode ser melhor do que aquela obtida para a norma uniforme, devido aos requisitos de regularidade dos dados da equação (2.6) serem menores.

Em [19], é apresentada uma análise de convergência do MCP para a norma uniforme, considerando a equação (2.1) com condições de fronteira homogêneas, $a_{1}=0, \varkappa=1 \mathrm{e}$ $a_{2}(x) \neq 0$. A estimativa para a ordem de convergência obtida, é restrita a $1 / 2$. Aqui, foi demonstrado que mesmo quando os dados da equação são mais gerais, a estimativa pode ser bastante melhorada, podendo chegar a 5/2, aproximando-se mais da estimativa observada na prática, conforme pode ser constatado pelos exemplos do Capítulo 5. 
A análise apresentada em [5], mostrou-se mais restritiva quanto a regularidade imposta nas funções $f$ e $l$, que devem pertencer a um espaço de funções menos abrangente que o considerado aqui. Isto leva em alguns casos, a uma estimativa para a ordem de convergência menor que a possível de ser obtida pela análise apresentada aqui, conforme discutido no Capítulo 4. 


\section{Referências Bibliográficas}

[1] Abramowitz, M.; Stegun, I. A.: Handbook of mathematical functions with formulas, graphs, and mathematical tables. Dover Publications Inc., New York, 1964.

[2] Atkinson, K. E.: A Survey of Numerical Methods for the Solution of Fredholm Integral Equations of Second Kind. Society for Industrial Applied Mathematics, Philadelphia, 1976.

[3] Capobianco, M. R.; Junghanns, P.; Luther, U.; Matroianni, G.: Weighted uniform convergence of the quadrature method for Cauchy singular integral equations. Singular Integral Operators and related topics, 153-181, Oper. Theory Adv. Appl., v.90, Birkhäuser Verlag, 1996.

[4] Capobianco, M. R.; Criscuolo, G.; Junghanns, P.: A fast for Prandtl's integrodifferential equation. J. Comput. Appl. Math. 77 (1997), no. 1-2, 103-128.

[5] Capobianco, M.R.; Criscuolo, G.; Junghanns, P.; Luther, U.: Uniform convergence of the collocation method for Prandtl's integro-differential equation. ANZIAN J. 42 (2000), no. 1, 151-168.

[6] Cuminato, J. A.: Numerical solutions of Cauchy integral equations and applications. Wolfson College, Oxford, 1987.

[7] Cuminato, J. A.: On the uniform convergence of a collocation method for a class of singular integral equations. BIT 27 (1987), no. 2, 190-202.

[8] Cuminato, J. A.: Uniform convergence of a collocation method for the numerical solution of Cauchy-type singular integral equations: a generalization. IMA J. Numer. Anal. 12 (1992), no. 1, 31-45. 
[9] Ditzian, Z.; Totik, V.: Moduli of Smoothness. Springer Series in Computational Mathematics, 9. Springer-Verlag, New York, 1987.

[10] Isaacson, E.; Keller, H. B.: Analysis of numerical methods. Dover Publications Inc., New York, 1994.

[11] Ioakimidis, N. I.; Theocaris, P. S.: On the numerical solution of singular integrodifferential equations. Quart. of Appl. Math. 37 (1979/80), no. 3, 325-331.

[12] Junghanns, P.; Luther, U.: Cauchy singular integral equations in spaces of continuous functions and methods for their numerical solution. J. Comp. Appl. Math. 77 (1997), no. 1-2, 201-237.

[13] Kalandiya, A.I.: Mathematical methods of two-dimensional elasticity. Translated from the Russian by M. Konyaeva [M. Konjaeva]. Mir. Publishers, Moscow, 1975.

[14] Luther, U.: Uniform convergence of polynomial approximation methods for Prandtl's integro-differential equation. Preprint 99-11 of the Dept. of Math. of the Univ. Chemnitz (Germany) (1999).

[15] Mastroianni, G.: Uniform convergence of derivatives of Lagrange interpolation. Orthogonal polynomials and numerical methods. J. Comput. Appl. Math. 43 (1992), no. $1-2,37-51$.

[16] Mastroianni, G.; Milovanovicć, V. G.: Interpolation Process. Basic theory and applications. Springer Monographs in Mathematics. Springer-Verlag, Berlin, 2008.

[17] Monegato, G.; Strozzi, A.: On the form of the contact reaction in a solid circular plate simply supported along two antipodal edge arcs and deflected by a central transverse concentrated force. Dedicated to Piero Villaggio on the occasion of his 70th birthday. J. Elasticity 68 (2002), no. 1-3, 13-35.

[18] Muskhelishvili, N. I.: Singular integral equations. Boundary problems of function theory and their application to mathematical physics. Translation by J. R. M. Radok. P. Noordhoff N. V., Groningen, 1953. 
[19] Nagamine, A.; Cuminato, J.A.: A collocation method for solving singular integrodifferential equations. BIT 50 (2010), no. 3, 657-688.

[20] Nevai, P.: Orthogonal Polynomials. Mem. Amer. Math. Soc. 18 (1979), no. 213.

[21] Rivlin, T. J. : An introduction to the approximation of funtions. Blaisdell Publishing Company, New York, 1969.

[22] Szegö, G.: Orthogonal Polynomials. American Mathematical Society Colloquium Publications, Vol. 23. Revised ed. American Mathematical Society, Providence, R.I. 1959.

[23] Theocaris, P. S.; Tsamasphyros, G.: A numerical solution of singular integrodifferential equations with variable coefficients. Appl. Math. Comput. 15 (1984), no. 1, 47-59. 1984.

[24] Thwaites, B.: The aerodynamic theory of sails. I. Two-dimensional sails. Proc. Roy. Soc. London 261 (1961), no. 1306, 402-422. 